Florida International University

FIU Digital Commons

FIU Electronic Theses and Dissertations

University Graduate School

3-11-2005

\title{
The impact of information technology on
} organizations : a study of enterprise resource planning system influences on job design and organizational culture

Susan Yvonne Clemmons

Florida International University

DOI: $10.25148 /$ etd.FI14060855

Follow this and additional works at: https://digitalcommons.fiu.edu/etd

Part of the Business Administration, Management, and Operations Commons

\section{Recommended Citation}

Clemmons, Susan Yvonne, "The impact of information technology on organizations : a study of enterprise resource planning system influences on job design and organizational culture" (2005). FIU Electronic Theses and Dissertations. 2385.

https://digitalcommons.fiu.edu/etd/2385 
Miami, Florida

THE IMPACT OF INFORMATION TECHNOLOGY ON ORGANIZATIONS:

A STUDY OF ENTERPRISE RESOURCE PLANNING SYSTEM INFLUENCES ON

JOB DESIGN AND ORGANIZATIONAL CULTURE

A dissertation submitted in partial fulfillment of the

requirements for the degree of

DOCTOR OF PHILOSOPHY

in

BUSINESS ADMINISTRATION

by

Susan Yvonne Clemmons

2005 
To: Dean Joyce J. Elam

College of Business Administration

This dissertation, written by Susan Yvonne Clemmons, and entitled The Impact of Information Technology on Organizations: A Study of Enterprise Resource Planning System Influences on Job Design and Organizational Culture, having been approved in respect to style and intellectual content, is referred to you for judgment.

We have read this dissertation and recommend that it be approved.

Ronald M. Lee

Sarah Mahler

Steve Zanakis

Irma Beccera-Fernandez

James R. Ridley

Joyce J. Elam, Major Professor

Date of Defense: March 11, 2005

The Dissertation of Susan Yvonne Clemmons is approved.

Dean Joyce J. Elam College of Business Administration

Dean Douglas Wartzok University Graduate School

Florida International University, 2005 
(C) Copyright 2005 by Susan Yvonne Clemmons

All rights reserved. 


\section{ABSTRACT OF THE DISSERTATION}

THE IMPACT OF INFORMATION TECHNOLOGY ON ORGANIZATIONS:

\section{A STUDY OF ENTERPRISE RESOURCE PLANNING SYSTEM INFLUENCES ON}

\section{JOB DESIGN AND ORGANIZATIONAL CULTURE}

$$
\text { by }
$$

\section{Susan Yvonne Clemmons}

Florida International University, 2005

$$
\text { Miami, Florida }
$$

\section{Professor Joyce J. Elam, Major Professor}

The primary purpose of this research is to study the linkage between perceived job design characteristics and information system environment characteristics before and after the replacement of a legacy information system with a new type of information system (referred to as an Enterprise Resource Planning or ERP system). A public state University implementing an academic version of an ERP system was selected for the study. Three survey instruments were used to examine the perception of the information system, the job characteristics, and the organizational culture before and after the system implementation. The research participants included two large departments resulting in a sample of 130 workers. Research questions were analyzed using multivariate procedures including factor analysis, path analysis, stepwise regression, and matched pair analysis.

Results indicated that the ERP system has introduced new elements into the working environment that has changed the perception of how the job design characteristics and organization culture dimensions are viewed by the workers. The 
understanding of how the perceived system characteristics align with an individual's perceived job design characteristics is supported by each of the system characteristics significantly correlated in the proposed direction. The stronger support of this relationship becomes visible in the causal flow of the effects seen in the path diagram and in the step-wise regression. The perceived job design characteristics aligning with dimensions of organizational culture are not as strong as the literature suggests. Although there are significant correlations between the job and culture variables, only one relationship can be seen in the causal flow.

This research has demonstrated that system characteristics of ERP do contribute to the perception of change in an organization and do support organizational culture behaviors and job characteristics. 


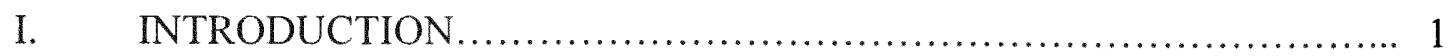

II. LITERATURE REVIEW .......................................... 5

Case Study Review................................................. 10

Enterprise Resource Systems...................................... 10

Successful ERP Implementations................................... 14

Failed ERP Implementations....................................... 26

Summary of Case Study Research Findings............................ 31

Summary of Literature Review...................................... 36

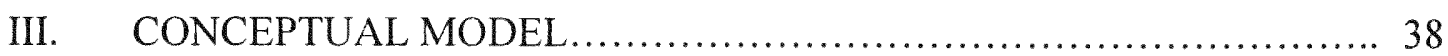

Information Systems ................................................. 39

Job Design.......................................................... 40

Culture ........................................................... 42

IV. RESEARCH DESIGN AND METHODOLOGY ........................ 48

Questionnaires................................................. 49

Detailed Hypothesis................................................ 63

Method................................................................. 91

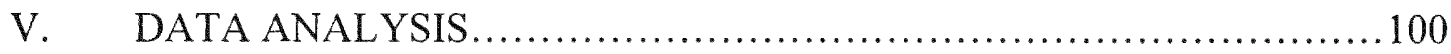

First Measurement.................................................. 100

Second Measurement............................................. 112

VI. DISCUSSION...................................................... 129

Legacy Environment............................................. 129

ERP Environment.............................................. 136

Behavior Changes.................................................. 139

Differences........................................................ 142

Limitations........................................................ 147

Future Directions ............................................... 148

Conclusions.................................................. 148

LIST OF REFERENCES .................................................... 151

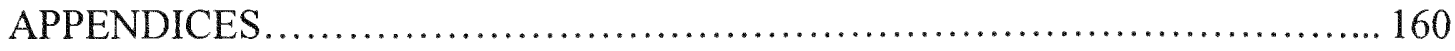

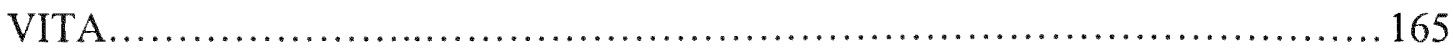




\section{LIST OF TABLES}

TABLE

PAGE

1. Organizational Responses by Case Study............................... 35

2. Cronbach's Alpha by TTF and JDS Constructs....................... 54

3. Demographics of Survey Participants.................................. 97

4. Participation by Department........................................ 98

5. TTF Component Loadings........................................... 102

6. Means of Summed Measurements from First Measurement................ 103

7. Pearson Correlations of TTF and the JDS for the First Measurement....... 104

8. JDS Component Loadings....................................... 105

9. Factor Correlations for TTF and JDS............................... 106

10. JDS and OCI Correlations for the First Measurement.................... 107

11. OCI Components Loadings....................................... 108

12. JDS and TTF with the OCI Factor Correlations for the First Measurement... 109

13. First Measurement Regression Models............................... 111

14. Means of Summed Measurements from Second Measurement............. 114

15. TTF Components - Second Measurement.......................... 114

16. JDS Component - Second Measurement........................... 116

17. JDS and TTF Correlations for the Second Measurement................ 117

18. Component Correlations TTF and JDS............................ 118

19. JDS and OCI Correlations in the Second Measurement.................. 119

20. OCI Component Loadings - Second Measurement..................... 119

21. Correlations of All Factors - Second Measurement..................... 120 
22. Step Wise Regression for Second Measurement........................ 122

23. Matched Pair Analysis.............................................. 124 


\section{LIST OF FIGURES}

FIGURE

PAGE

1. Conceptual Model.................................................... 39

2. Schein's Model of Organizational Culture ................................ 45

3. Detailed Model of System and Job Characteristics Survey Variables........... 79

4. Detailed Model of Job Characteristics and Culture Dimensions Variables....... 81

5. First Measurement Path Analysis......................................... 110

6. Second Measurement Path Analysis..................................... 121 
I. Introduction

The adage that "information technology possesses the ability to transform" is commonplace in the business environment. Transformation is an expected result of a successful information technology implementation and usually a stated objective justifying the cause (Robey and Sahay, 1996). Most of the research studies to date have examined "transformation" in terms of the impact of information technology on organizational design and performance and the role that it plays in gradually changing the organization (Yates and Van Maanen, 2001). What has been largely ignored in these studies is the effect of information technology on shaping organizational norms and behaviors generally referred to as organizational culture. This is important because technology has the potential ability to be a strong lever for culture change (Laurila 1997; Cummings and Worley, 1998).

Organizational culture can be thought of as a set of assumptions and histories that program organizational responses and action, and shape the organizational member's thinking and behavior (Schein, 1985). Work processes embody these programmed actions and act as supporting structures to recreate the culture. Information systems are a critical and key structural component of today's organizations that fundamentally shape the way an organization accomplishes its work. Is it possible that a new information technology implementation, through the changes in organizational and work processes, can significantly impact the norms and behaviors of the organization? This is the fundamental question addressed by this research. In particular, this research addresses if and how a new information system, in the form of enterprise information systems (or enterprise resource planning-ERP), affect an employee's perception of their job, and if this in turn 
influences the perception of said employee's view of organizational culture. This research measures the employee's perception of job and organizational culture prior to and after the implementation of the new information system. This allows a deeper understanding of exactly how an ERP information system changes job and organizational culture perceptions.

Examining information systems as a cultural-cognitive ${ }^{1}$ element of institutions that influence and reinforce culture can bring new insights involving the impact of the information system in the organizational environment. The ability to anticipate how new information technology might affect the existing organizational norms and behaviors would be invaluable to organizations implementing new information technology.

Information technology can be a powerful enabler of organizational change since the implementation of a new system destabilizes or destroys the old orientation and replaces it with another one. This is not necessarily the case with other change initiatives. As executives, managers and administrators attempt to significantly change the organization, the inability to change the underlying ideology or culture can ultimately cause the change initiative to fail (Meyer and Starbuck, 1993). Though aspects of the new ways may linger, most organizations resort back to their original ways of operation by overpowering the change and rejecting the new orientation for the comfort of the old patterns. According to Burke and Biggart (1997), 75 percent of business mergers and acquisitions fail at this type of strategic change.

\footnotetext{
${ }^{1}$ Cultural-cognitive elements are shared conceptions that constitute the nature of social reality and the frames through which meaning is made. They are a central theme of institutionalism.

Scott, R. W. (2001). Institutions and Organizations. Thousand Oaks, CA, Sage Publications, Inc.
} 
Reverting back to the original patterns of behavior is made impossible when a new information system is implemented. Therefore, a better understanding of the linkages between technology and culture becomes very important in order to increase awareness and knowledge about how to affect organizational change. This knowledge would allow institutions to design organizational structures and processes made possible by the technology that supports the desired new cultural behavior. As a result, managers would be able to better direct organizational change and enhance the surroundings to sustain that change.

This research examines an organization before and after the implementation of an ERP system at a public state University. In particular, this study seeks to uncover if and how the information technology affects an individual's ${ }^{2}$ perception of his or her job requirements and how this in turn affects organizational culture when the information technology is a collection of legacy software applications and when the information technology is a new ERP system. Comparisons of these relationships in both time periods are examined. The results are intended to help the leadership of an organization understand and ultimately manage a changed culture resulting from the implementation of an information technology solution, such as an ERP.

This research draws on two main streams of research. First, studies that focus on how the implementation of technology modifies an organizational culture through its impact on organizational variables such as work design are examined. This examination will provide support for the claim that information technology, through its impact on job

\footnotetext{
${ }^{2}$ An individual here refers to a key system stakeholder - typical stakeholders within an ERP system would include transaction processors and decision makers in Human Resources, Finance and Information Technology.
} 
design, changes the organizational culture. Second, recent case studies of organizations using ERP information systems are reviewed in order to identify common themes and activities supporting this claim of change. Ultimately, the conceptual model that underlies this research study is supported by both the literature and case study review. 


\section{Literature Review}

The notion that the introduction of technology in work settings transforms productivity, organizational processes, and effectiveness is widely accepted. When examining more closely how technology transforms organization, it is necessary to base one's investigation on the linkage between technology, organization structure, and organizational culture. Structure refers to the ordering and coupling of components used to create an organization (Georgopoulos, 1996). Within organizations, structure manifests itself along several dimensions, including work design, centralization of authority, hierarchy of influence, and the degree of role specification. These dimensions - making possible or rewiring certain types of behaviors and ruling out or making difficult other types of behaviors - shape the operating culture.

Leavitt and Whisler (1958) proposed a useful institutional framework of structure, people, tasks, and technology. He demonstrates that changes in the organization's structure may drive changes in tasks, technology, and personnel. When any one of these constructs is affected, it in turn affects the others. One way to create structural changes may be to change the technology in the organization (Lucas and Baroudi, 1994). Structural change impacted by information technology would also impact the organizational operating culture.

Over the past four decades, many organizational theorists have postulated the relationship between technology and organization structure. The results of this research have produced contradictory and sometimes confusing findings about this relationship (Fry and Slocum, 1984). For example, Woodard (1965) found that technological characteristics shaped the structure of English manufacturing companies. In a later study, 
however, Hickson, Pugh, and Pheysey (1969) found little evidence to support a technology and organization relationship. Many other comparative analysis studies by Blau and Shoenherr (1971), Child and Mansfield (1972), and Hage and Aiken (1967), drew general conclusions across many organizations and industries, but are limited on explanations (Gerwin, 1981).

The linkage between technology and organizational design and decision-making also has been extensively explored by organizational structure researchers. That technology influences the work task is a logical expectation, which has been validated by numerous research studies. Using technology has a high level of impact on the job design, job complexity, and job interdependence (Cooke and Szumal, 2000).

George Huber set forth a theory of effects that decision-aiding technologies have on organizational design, intelligence, and decision-making (Huber, 1990). He discussed several organizational traits that may be influenced by the use of "advanced information technologies" in the organization. Specifically addressing organizational decision-making and technology, he proposed several interesting relations. The first was the way in which the use of computer-assisted communication technologies leads to a larger number and variety of people participating in the making of a decision. He proposed that due to the ease of information gathering and flow across the organization that the technology would enable, more people would be able to gain access to the information and the decisionmaking process. Another function he attributed to decision-support technologies was the ability to reduce the decision-making time and enable higher quality decisions for the organization. Although he never addressed it directly, he was referring to a new, evolving breed of computer-assisted technologies that would "fit in the domain of the organization 
theory." Though controversial for his time, many of the aspects of his theory have been supported with further research (Leidner, Carlsson, et al., 1999).

While much research has shown that technology impacts job design, the nature of this impact depends on how the organization assimilates the use of the technology into its day-to-day routines.

A classic study in this area is Barley's (1986) institutional study of radiology departments for two community hospitals. He examined changes occurring over one year in the social structure of radiology departments. The same technology was implemented in two different departments within the same hospital: technology was seen as a disruption to normal established behavior, and the disruption created an opportunity for new behaviors and routines to take hold in the settings. The two departments created different ways to integrate the new technology. In both cases, however, decision-making became more decentralized in the individual work roles. It was the "technology occasion," not the technology itself that led to the changes that occurred.

Manning (1996) studied the use of cellular phones in police organizations and found that technology not only had practical applications, but also that the technology possessed the ability to reshape work processes and authority patterns within organizations. He argues that technology can be viewed as both a tool and a symbol because it focuses attention on the differentiated cultural meanings and on the uses such technology has for members of the organization.

In a study of the Stealth bomber project (Argyres, 1999), the NASTRAN system (an advanced structural engineering software used to perform analysis of large structures such as buildings, bridges, and military aircraft) acts as a replacement for organizational 
structure to facilitate project coordination. The system transformed the design work in such a way as to radically decrease the amount of engineering effort the parties required. The author states, "The combination of direct and interaction effects observed in this project may generalize to other settings and other types of information systems...and suggest plausible mechanisms by which IT can substitute for hierarchy" (Argyres, 1999, p. 164).

In an examination of how technology changes organizational work activities over time, Orlikowski (2000) looked at the activities of technical support employees in a large U.S. software firm over a two-year period. The technology being implemented was a customer call-tracking system developed in the groupware Notes (from the Lotus Development Corporation and later acquired by IBM). The system tracked customer technical issues and supported the resolution process. The successful adoption of this system was attributed to the informal ways in which the employees themselves fashioned their uses of the technology rather than managerial or technological imperatives. The result was an unplanned transformation of the support organization. The work of technical employees increased in collaboration, documentation, responsiveness to customers, accountability to management, and productivity. Just as the other three studies demonstrate, technological transformation within an existing structure was not a direct result of the introduction of technology, but of a more complex combination of individual and organizational social responses.

Although usually unplanned, culture change is a necessary ingredient of successful technological inspired transformations. The change is seen as a process, not as a single event, that is linked to several organizational disturbances to create a new 
operating environment. Barley's (1986) use of institutional theory to demonstrate the creation of new norms for the organization implied a change in culture. This culture change also was apparent in the Orlikowski (2000) study. These findings confirm that the use of technology depends on greater organizational influences. However, the studies ignore the notion that technology directly reinforces the organizational setting through the shaping of task behaviors. Mutual social sense-making to perceive the technology in other ways is a product of individual sense-making, which is influenced by the technology through structure. The indirect effect of the technology usage can be supportive, disruptive, or neutral to the organizational setting (or culture).

The theme that seems to be missing in organizational change involving technology is using technology as a reinforcement of structure for the institution. Technology is seen as an occasion for change; however, its impact on culture may be considered as an aftereffect. Orlikowski's (2000) study demonstrates how the changing of norms and behavior can evolve over time using technology; however, changing the normal behavior of the organization was not a direct intention of the technology. In the next section, more support for this understanding can be found in studies of information system users. 
Although the literature alludes to the impact of technology on organizational change, there are no specific references to components of the organization or the technology that can be directly connected to the change. In order to understand linkages between specific technologies and outcomes in the organization's behavior, a narrower view of the phenomenon is necessary. An examination of linkages between similar technologies and organizational responses is necessary to develop a conceptual model of technology's influence on an organization's culture. The most popular system-wide application package currently used by large organizations is an enterprise resource planning (ERP) system. A fully functional enterprise-class package, ERP may be considered the core operational infrastructure supporting all basic business transactions (Sprott, 2000). Using this system has fundamentally changed the way organizations work. Due to their "transformational qualities," these types of systems will be the technology component in the study.

In the following section, a brief description of ERP systems is presented followed by a review of ERP case studies. Linkages between the implementation and changes in organizational behavior are sought. These elements will form the basis of the conceptual model that underpins this research.

Enterprise Resource Systems

Historically speaking, microprocessor and computer chips evolved during the fourth generation of computers, making it possible to process large amounts of information. As technology's hardware was getting smaller and faster, the information 
processing abilities of the software was growing exponentially. It was not until the current generation of business computing that complex organizational behaviors such as coordination, workflow, and governance were appearing as common system functionality in large software applications.

The ERP concept of systems was to merge several visions or functions of control and accompanying practices embedded in software systems (Koch, 2001). The system functions are interwoven with each other to deliver the necessary work practices and controls. ERP systems can be viewed in economical, logistical, and informational terms. The economical vision sees the enterprise as a financial entity, and it is related to accounting practices. Accounting principles and routines, such as internal control of cost centers, and activity-based costing, are practical examples. Logistical visions refer to the material flow through the organization or enterprise. The technology of manufacturing resource planning (MRP) offers an interpretation of these types of routines. The last vision, informational, sees the enterprise surrounded by the information technology platform. Within the visions, functional gaps were filled with additional software processes to complete a holistic support vision.

Traditionally, the approach to information systems business support was to align systems to functional areas and user tasks. This emphasis on functions encourages vertical systems with their own proprietary data, software, and technology components (Cook, 1996), where each system is responsible for productivity within the function, and not the complete enterprise. In contrast, the ERP computing approach focuses on the complete set of processes necessary to support the entire business operation. This radical 
shift from vertical to horizontal orientation is the critical difference in enterprise applications. The functional areas are viewed as seamless, allowing processes to flow across the boundaries and be used and controlled by the entire organization. The control of process is through sets of business rules determined by the process owners through an activity referred to as system configuration. ERP systems present a pre-constructed set of processes to represent a type of industry or common practice (Fleck, 1993). It is within this pre-constructed template that the organization must choose the best representation of their process activity.

The design of these systems creates distinctly different computing and working environments for the organization. The main characteristics of ERP that are cited as unique are integration (or concurrency), data concentration, and a forced decision-making hierarchy. Each of these adds an element of complexity to the operation of the system and the users understanding of it.

In an ERP system, all aspects of the business are connected via one database, one application, and one common interface across the organization. It is designed to process information remotely and then "ripple" the results to the rest of the organization through common processes (Bingi, Sharma, et al., 1999). This type of integration allows sharing of information in a standard format across many departments regardless of physical, language, or currency differences. The use of ERP amplifies the importance of data and process concurrency by eliminating organizational boundaries and other artificial barriers. If designed correctly, the computer process does not rely on physical movement 
or human interaction to progress. The process relies on a computerized pre-programmed set of rules to connect and interact when necessary.

Another characteristic of integrated systems, referred to as data concentration, is the combination of separate records relating to the same subject into one related record. Integrated systems eliminate the process of multi-recording and transcribing data to update separate records with one single input to the computer record. This ability demands appropriate controls to facilitate timely coordination and scheduling of all processes (manual or otherwise) to be undertaken by the different departments responsible for the information, so that a single input to the computer system, also referred to as a single point of entry, is accomplished. Integrated systems thus join systems that traditionally have been kept separate and, by their very nature, cut across the conventional departmental boundaries that normally exist in a business.

The last main characteristic of ERP systems is that operational decisions are pushed down to a point as close as possible to the source of the event. Manual processes that normally require segregation of duties are often eliminated in an ERP environment. Work tasks that were separated into several departments may be combined into one job for entry into the system. This same effect can be seen at the organizational level. Responsibilities that had been divided among many sub-units of the organization may be concentrated into a single application system. This changes the work and accountabilities of sub-units or departments. The control or power structure of the organization may be affected by the changes imposed by the system processing. Holsapple and Sena (2003) found that the impact of this characteristic is present in many adopter organizations. The 
empirical study found that providing a repository of knowledge for solving problems and the mechanisms to facilitate communication within an organization were the two components that allowed lower level decision-making within the ERP environment.

Integration, concentration of data, and concentration of responsibilities gives ERP systems the ability to streamline the entire operations process. However, this efficiency comes at the price of drastic structural changes for organizations. Due to their hierarchical nature and complexity, ERP systems tend to inflict control upon the organization in the form of system rules and procedures in a pre-defined standard of infrastructure (Koch, 2001). This infrastructure jurisdiction controls not only the information processing of the system but also the work tasks associated with the system for information retrieval and dissemination in the organization. The system can be modified to reflect the structure of the organization; however, it must not be altered beyond a certain controlled boundary. If the package cannot be adjusted to fit the organization, then the organization has to adapt to the package and change its procedures (Bingi, Sharma, et al., 1999).

\section{Successful ERP Implementations}

This section will compare case studies of organizations seeking change that have used or contained the component of ERP technology to support the effort. In these studies, the organizational change objective was a result of three common strategies: mergers and acquisitions, spin-off or diversification, and operational improvement. Radical change was experienced not as result of the system implementation but due to the organizations seeking changes in organizational behavior and, in some cases, to survive. 
References to change in organizational behavior and specific corresponding system functionality or characteristics that enabled the behavior modifications are found to support the thesis of information systems acting as structural influences on organizational behaviors. Multiple sources of information were used to confirm the setting and organizational results of the transformation. The finding of behavior modifications in the case studies provides the evidence to support the claims of this research.

ERP in Mergers and Acquisitions

Dealing with human behavior attitudes is the most cited issue in business merger management (Buono, Bowditch, et al., 1985). The significance of the change in attitude and behavior by the worker has been highlighted by research that found that despite favorable strategic and operational compatibility, mergers have less than a fifty-fifty chance of being successful (Louis, 1982; Pritchett,1985). Technology-induced change can also support human behavioral changes as found in the following case of Rolls Royce. Although radical change was triggered by the acquisition of additional facilities and a change in the global marketplace, the recognition and development of new organizational behaviors can be partially attributed to the deployment of enterprise technologies.

During the late 1980s, Roll Royce consolidated and increased its industrial power, aero propulsion, and industrial gas turbine capabilities with the acquisition of Northern Engineering Industrial and Allison Engine Company. With facilities in more than fourteen different countries, the complex, independent business of each product line was too cumbersome to be managed separately. Rolls Royce decided to make radical changes 
to their business in response to increased market orders and take advantage of a new type of information system, ERP, being used by an American facility. Accurate information and direct communication is vital to the logistics processes, and their current technical and organizational structure was not able to accommodate the changing business environment (Yusuf, Gunasekaran, et al., 2004).

Yusuf and colleagues' case study of Rolls Royce mentions several organizational behavior changes that resulted from the use of the system. Management recognized the need for a shift with the new operations. The new operational training, attended by all employees, went beyond the boundaries of the work and included cultural and organizational issues. Management thought that if employees were armed with the additional understanding of the new approach to business, they would embrace the changes. Organizational behavioral modification was assumed with the success of the new system usage due to the changes the information technology introduced into the work setting.

New roles, such as MRP controller and capacity owner, and behaviors were designed. Workers were tested, selected, and trained to fill the new job responsibilities. From the case study, an implementation team member speaks about the role change (Yusuf, Gunasekaran, et al., 2004):

We had to go through symmetric tests, aptitude tests, interviews, it was quite daunting really, I mean to think that here I am, quite settled but I've got to make these moves. I'm told that I've got to spend X amount of time around PO, it's a bit strange as I must spend around 80 percent of my time on the shop floor today, and that's going to change dramatically (261).

Increased organizational trust in information and increased customer confidence are other behavior influences that resulted directly from using the new system 
functionality. Because the system reinforced data accuracy through a centralized standardized database, the chance for the data to be incomplete, untimely, or inaccurate went away. The new system monitoring process provided work visibility for the entire organization, improving the internal confidence in executing and completing work on time. The new ERP system reinforced the shift in operations that was necessary to align the new businesses and their respective organizational behaviors. It helped to establish the organizational confidence and trust to support the change.

\section{ERP in Spin-off or Diversification}

The Enterprise Network System business unit of AT\&T had been through several transitions since 1983, including the acquisition of legacy business structures and strategies over the years to support the communications giant. In 1996, the unit was spun off from AT\&T with the creation of Lucent Technologies (Francesconi, 1998). It was during this time that management decided to redesign the existing business processes, people practices, and legacy systems to support the renewed business operations, customers, and shareholders (Cowan and Eder, 2002). A project to address these issues was started and completed in late 1999, and a fully functional ERP system was used as the technology component enabler. In October of 2000, Lucent spun off Avaya, a portion of the wire division and its network systems business unit. Avaya was a virtual company with key business functions in R\&D, marketing, services, channel management, and contract management. The glue holding Avaya together was contracts and the integrated ERP system. 
Overall, the system-designed management of process influenced the company's organization structure where flexibility, adaptation, and responsiveness became the drivers of the new structure and culture (Dwyer, Heralcleous, et al., 2003). Avaya required the creation of corporate headquarter functions and the infrastructure to support their operations. The focus of how the functions were created depended on the existing functionality built into the information system technology infrastructure and less on the creation of new business processes and people practices. The system created an operational blueprint for the business to build upon. Avaya put in place standard global business processes and global people processes, and the technological structure was flexible enough to be upgraded as the business strategy evolved (Cowan and Eder, 2002). By incorporating key system characteristics - such as standardization and data integrity into their operating and people design, the company was able to start as a success ${ }^{3}$. Significant legacy organization cultural and structural barriers had to be overcome for the change to be successful (Dwyer, Heralcleous, et al., 2003).

The case of Avaya is unique in that the system was the stable influence during the change crisis. The system provided the structure for organizational behaviors that Avaya wanted to retain in the emerging organization. Establishment of structure is an important characteristic for organizational change to be successful.

ERP in Operational Improvement

Many organizations cite operational efficiency and improvement as the rationale for change or for transformation. Poor organizational performance in the marketplace has

\footnotetext{
${ }^{3}$ In 2000 , the Avaya facility in Ireland was awarded the European Quality Award for continuously satisfying customer needs.
} 
negative effects not only for the organization but also for all individuals in the organization, because their intrinsic rewards are diminished as are their security and financial rewards. When an organization's performance falters, there will be a high level of dissatisfaction with the existing management paradigm (Lawler, 1988). The redesign of business processes enabled by technology can improve operations, and it can also be the byproduct of an information system implementation as the organization tries to accommodate the system characteristics and functionality. In the following six cases organizations have promoted transformation by leveraging an information technology system. While the system is not the trigger, it is part of the solution to change the current operating environment. Common to each of the cases is the desire to modify an organization's behavior.

Owens Corning

Owens Corning is the leading producer of fiberglass-based building materials. In 1994, Owens Corning needed to adopt a stronger market orientation due to investor complaints and unmet customer requirements. The company surveyed the management employees to understand their perception about the company, and the results showed that it was in the bottom third of peer companies for overall ratings (Day, 1999). The president led the change program with a multifunctional task force and the corporate board. The vision was to promote "system thinking" - a concept that products are all systems that work together to solve customers' problems. All connecting elements of processes, systems, and roles were changed to support this new culture. Day (1999) discussed this change as such: 
A centerpiece of the change process at Owens Corning was the realignment of systems to support its new strategy...Owens Corning replaced all systems...they [the systems] had to be completely redesigned to mesh with the new processes that flowed information rather than leaving it in separate silos... [After the system implementation] it aligned and integrated external information flows and internal processes and information (20).

Although the implementation of the system had some slowdown due to structural realignments and the learning curve for workers, an unanticipated impact was to longterm customers. It was such a dramatic, unexpected shift from the usual business process for many customers, and due to lack of communication about the change, many angry customers left for other suppliers (Koch, 1999). The "revolution" changed Owens Corning and it adopted the vision phrase as the name of its corporate organization. The culture embraced the complete customer experience concept and positioned all manufactured components to fit this strategy from contractors to homeowners (Caldwell, 1998). The ERP implementation resulted in various changes in design and structure to the organization indicating that technology dictated the organizational design and structure for the renewed company. The key system characteristics reflected in the organization's behavior are centralized coordination among various functions, divisions, and countries (Palaniswamy and Frank, 2000), dispersed decision-making (Rajaagopal, 2002), and standardization.

Texas Instruments

Market forces compelled Texas Instruments (TI) to make radical shifts to its business in 1996. The business wanted to focus on meeting customer needs in a uniform fashion, but still allow flexibility to respond to changing needs, as the company could no longer afford to have ad hoc processing to accommodate the customer (Sarkis and 
Sundarraj, 2003). The goal of the technology structure was to be open and allow use of the Internet, as they wanted to change the business from a local response to a unified global effort in the marketplace.

To support these goals, TI implemented a single ERP global model of business. All the processes were connected and supported one effort. The technology forced standardized processes and one data structure. From the Sarkis case study:

The success of the single-instance, integrated, global model has fundamentally transformed how business is conducted at TI. It has allowed the company to have actions taken, say, in the US and determine the impact on other parts of the world (439).

The internal operations were opened through the Internet to customers and vendors to allow visibility and enable communication flow. The technology enabled the organization to see itself as a global, unified, coordinated effort. Besides process standardization, the case study also alludes to increased performance monitoring and coordination behavior as direct results of the new business model and the new system. The technology contributed to the organizational transformation as enforcement and reflection.

Eastman Kodak

To compete globally, the Eastman Kodak Company wanted to use one operations model for all its businesses. The technical infrastructure of the legacy systems included 2,600 software applications, 4,000 system interfaces, and 100 programming languages all running on an old mainframe system (Palaniswamy and Frank, 2000). The supporting technologies and work processes needed to be aligned to execute a single business approach. An initiative was launched to reduce all the company's applications and 
interfaces down to one integrated system that was operating one version of corporate data and work processes.

Kodak approached the system replacement as a complete re-engineering of the processes and the business. Before the start of the ERP project, a model was developed based on the system functionality and how the business needed to be conducted to support the global goal. The senior managers of business units and major functional organizations were responsible for the creation and implementation of the new work processes and system.

Like Rolls Royce, as a part of their redesign effort, Kodak redefined roles, responsibilities, and reporting structures to better fit system functionality during the deployment of an ERP system (Anonymous, 1996). With extensive user training and an online quick reference tool, the company also invested heavily in the support of the new organizational operating work behaviors that were being created by the system. The focus of the support was to create an understanding for the new behaviors in a work context. From an industry trade article, a manager comments on new organizational behavior (Stevens, 1997):

We recognize that as we go forward there will be a requirement for ....adherence and compliance to standards that are global. These are not the normal behaviors for regional organizations. In the regions, they have had a lot of autonomy so there's going to be a lot of learning (35).

Kodak was able to leverage its knowledge in new product development and apply the disciplined "phase and gates" competency to the ERP project. The company treated the new system similar to a new product going to market and managed the launch and support efforts as such. These institutional behaviors of new product launching were 
applied to the benefit of the system project. Key system characteristics, which were absorbed and are now part of the organization's core behavior, included integration and standardization (Stevens, 1997). Although the corporate culture was not the target of the change, the system processing and characteristics changed the behaviors of the organization and thus influenced a change in the organizational operating culture.

\section{Viskase Corporation}

Due to increasing competition, the Viskase Corporation recognized that its manufacturing processes needed improvement. A study by Coopers and Lybrand consultants found the lead-time for orders was very high. Also, based on a customer's request, completed orders were held in inventory for up to nine months. The customers were not billed for the material until it was moved out of the inventory. Viskase, a leading manufacturer of cellulose casings for the food industry, had become a warehouse service without knowing it. In an effort to re-organize the business and improve manufacturing processes, Viskase implemented an integrated ERP system that embedded all the functionality of the material resource planning (MRP) processes. Viskase knew that these types of systems would be beneficial in reducing lead times and inventory, as manufacturers have benefited from such MRP systems for over two decades. However, the implementation of an ERP system throughout the complete business has created other salient benefits resulting in changed organizational behavior (Rajaagopal, 2002).

Before the ERP implementation, only a few persons who interacted with the steps or information knew the process of forecasting and managing actual orders. These individuals were able to comprehend the information and develop plans for production 
and purchasing, whereas personnel were focused on their respective units or contribution. Then the system connected the organization from end-to-end and allowed related functions access to information that they required to work more efficiently. Complex business processes became both available and transparent to all members of the organization, enabling operations to be understood by the entire organization. A connection between individuals' roles and a shared understanding of their contributions was created. The impact on decision-making was apparent in timing - decisions were determined and executed faster. Although the intended impact of the system was to correct operational deficiencies, the actual impact spread across the organization and resulted in a workforce with more operational knowledge.

Diebold, Incorporated

Diebold, Incorporated manufactures banking technologies, such as ATM machines, for financial institutions and various retail outlets. Although their previous system and operational infrastructure enabled efficient and profitable workings, it had three critical deficiencies. The first is that it was unable to support a global product offering. A "product configurator" software model was needed to manage the diverse specifications warranted by myriad international local markets (Rajaagopal, 2002). Next, the system only updated in a nightly batch mode. This made the data outdated later in the day for decision-making. Finally, it was unable to support integration and communication among all locations in the supply chain. Organizational-level integration and timely data were important to support worldwide operations. The decision to move to an ERP system was made and executed. 
Initially, the organizational response to the new way of working was not enthusiastic. The new system required laborious data entry, yet once entered, the information was available for process analysis and decision-making. However, it took effort to move the organization from the old form of paper note-taking to the digital mode (Palaniswamy and Frank, 2000). The users who expected that the new system would lessen their workload were disappointed. In the first steps of the process, the data entry requirement was increased. Customer support jobs had to be modified to compensate for the additional workload. Workers had to be knowledgeable about the local configuration requirements of each customer's order to correctly interpret the proper product knowledge that prior to the change was held only in the sales staff who translated the requirements to the shop floor. Similar to the Viskase Corporation, Diebold experienced a transformation of worker knowledge as a result of the system implementation. This knowledge has helped to increase coordination and communication within the supply chain and assisted with better overall management of the process.

Failed ERP Implementations

Just as the system characteristics bring out new and improved organizational behavior as found in the successful ERP implementations previously described, they can also magnify organizational shortcomings and wound organizational cultures. The rate of change and increased workload on human resources can turn an implementation into a costly disaster for some organizations (Osterland, 2000). Many organizations refer to the learning involved with ERP implementations as "painful" due to the stress that it causes. Poor training and preparation of the core team of people who are running the business are 
cited as the primary reasons why companies experience failure and pain (Wheatley, 2000). Although inadequate training can contribute to the lack of understanding, organizations that fail to make the ERP transformation may also possess inert cultural behaviors that work against the system characteristics. The old behaviors do not change, as the introductions of new behaviors by the system fail to take hold and ultimately the organization does not realize the benefits of ERP systems. Due to the large cost of the system, most organizations cannot afford to completely abandon the new system and will make the appropriate organizational adjustments. However, these adjustments come after expending large amounts of resources. In the following section, three companies are examined for common reactions to failing ERP projects. Two of the organizations continued to complete the transformation and the third abandoned the effort.

\section{Nestlé USA}

The intent to implement an ERP system at Nestlé USA, the U.S. subsidiary of a Switzerland-based consumer goods giant, was the first step of the parent conglomerate's goal to transform 200 operating companies and subsidiaries in eighty countries into one global "machine" (Anonymous, 2001). The implementation would ultimately have taken six years and more than $\$ 200$ million dollars to complete. The system project was fraught with dead ends and costly mistakes. The basic issues at Nestlé USA were not the abilities of the technology but how the system characteristics affected the organization and working operating culture. In an interview with the CEO of the parent company, Peter Brabeck-Letmathe comments on the company's core values (Burrus-Barbey, 2000):

The most fundamental principle is that Nestle is a company oriented towards people (consumers, customers and employees), products, and brands, but not 
systems. We are not a systems-driven company...We use systems because we know they are necessary, but we are never driven by that system. As stated in our document, 'Nestlé is more people and product oriented than systems oriented.' Systems are necessary and useful but should never be an end in themselves (497).

Although the key management roles acknowledged the business process change necessary to accommodate the system, there was little understanding of the degree of change that would be involved. The head of technology, Jeri Dunn, commented in a trade magazine (Worthen, 2002):

Nestlé USA learned the hard way that an enterprise-wide rollout involves much more than simply installing software. When you move [to ERP], you are changing the way people work... You are challenging their principles, their beliefs and the way they have done things for many, many years (24).

Employee resistance to the work change also traced back to communication issues within the organization. Groups directly affected by the system characteristics were not part of the core ERP project team. The groups were "surprised" by the changes and rebelled against new processes. Instead of embracing the new forms of behavior, the comfort of the old ways seemed to dominate. From the CIO Magazine case study article:

Nobody wanted to learn the new way of doing things. Morale tumbled. Turnover among the employees who forecast demand for Nestlé USA products reached 77 percent; the planners simply were loathe or unable to abandon their familiar spreadsheets for the complex models [of ERP] (25).

The organizational communication problem also impeded the system characteristic of data and process integration. Interdepartmental linkages were built through common data and process. However, due to system issues, the integration component of the system was not engaged simultaneously, and it was up to the departments to coordinate and connect. Proper procedure executed in one department with no coordination to another was incorrectly managed in the next department. From the case study (Anonymous, 2001): 
"In its haste to unify the company's separate brands, the project team had essentially replaced divisional silos with process silos" (24).

After halting the project several times, the business started managing the change and involving the employees in the implementation dates. Departments were asked when new processes could be obtained and then the system deadlines were aligned. After a complete sign-off from all key users and a sufficient amount of change support was in place, the new processes, data, and system were asked to be used. Individual workers needed to understand the impact on their jobs before they agreed to change. The workforce adopted the new ways after the impact was analyzed, communicated, and understood. The organization modified its operating behaviors of old to support the new system characteristics.

\section{Soft Drink Bottler}

The decision that drove a major soft drink bottler to implement an ERP package came not from wanting to change its core business like Nestlé USA, but from trying to leverage ERP system features to enhance its current operations. The bottler employed a "do-it-yourself" attitude. The bottler rejected changing operations for the systems sake and did not gather outside opinions on the best way to use the new technology. The management saw the ERP system providing new ways to manage the business, but did not understand the amount of change necessary to conform to the system characteristics (Barker and Frolick, 2000).

Communication between each of the bottler's functional areas was not very high. The bottler organization did not have a formal communication process, and the project 
team only revealed details about how the system handled day-to-day activities after problems appeared. Each department acted without interaction, was accountable for controlling only their individual budgets, and did not share resources. The lack of formal communication between departments on key project decisions led to the dismissal of the first ERP project leader. Employee turnover started to climb as more of the employees anticipated no training or support due to a lack of communication about the new system. The project also lacked visible upper management support. Frustration and a "loss of control" were apparent as the implementation started forcing different sub-units to share knowledge.

The organization did not seek change as the system demanded new behaviors. A good example is the system characteristic of integration, which demands communication and coordination from the organization. To correctly connect the components, all of the flow steps and corresponding data must be documented. Without management reinforcing the need to work together, each department tried to control their own information. Standardization of data and of process did not occur.

The bottler used the ERP system but with the system characteristics turned off. Integration between each of the modules was not in place and no standard processes or data exist for the entire company. The system code was changed to reflect the current organizational structure and behavior patterns. Due to the inability of the organization to modify behaviors, the ERP package was not being used to its fullest capabilities. 
Similar to the last two case studies, a major manufacturer located in the Middle East wanted to radically change operations to support the movement into the global marketplace. Due to an aging technology structure, the decision was made to replace and redesign the operations with an ERP system. The technology was to act as a connecting agent, joining the four subsidiaries and the parent company to enhance current operations. According to Al-Mashari and Al-Mudimigh (2003):

Many problems plagued the project from the outset. The project manager allowed the boundaries of the project to keep increasing without regard for the resource or timing impact. Ownership of the change was not clearly driven by any one person or department. This resulted in departments making decisions without regard to other departments that may be impacted by the change. The new knowledge of the system was isolated by the systems group and not shared across the departments. This lack of communication created confusion and managerial distrust. Although the impact of the system was considered, the necessary daily operations adjustments to jobs and work tasks was expected to come from the shop floor. This is an impossible task without a complete understanding of all of changes (25).

The ERP project was deemed a failure when it did not return the benefits anticipated, went longer than planned, and cost more than was budgeted. Because of this failed experience, employees received a negative perception of re-engineering, and an increased sensitivity toward any change effort in the future. An attempt to correct management and the communication structure was abandoned. The firm rejected the new system characteristics because the changes lacked support and understanding. The operating environment had not been modified to accept the new way of working and was dependent on old organizational behaviors to support the change. 
Summary of Case Study Research Findings

The case review reveals several common elements that demonstrate the effects of ERP systems in organizations. These elements are grouped into two categories. The first grouping includes what the organization changed to in order to accommodate the system characteristics and is labeled as organizational structure. This action is taken to adjust the current operating culture to accept and reinforce the new system characteristics. In a classic non-ERP system implementation, this step would be reflected in system changes to accommodate the worker's job. System modifications would try to minimize the impact on the workforce. In an ERP implementation, the opposite action of the organization is required. Processes and jobs are redesigned to minimize system changes and take advantage of software-delivered characteristics.

The second grouping contains ways the organizations respond to the changes imposed by the system. These are labeled as organizational behaviors. Although some behaviors can be forecasted by the work routine, such as standardized outputs, the majority of behaviors are in response to how the organization incorporates the use of the new system characteristics into the daily operations.

The final assessment of the impact of the change is if the organization permanently adopts the new set of behaviors. Achieving a permanent change is critical for a successful transformation. Table 1 identifies each element, structure, behavior, and permanent change by case study. 
Organizational Structure

The changing of job design was the most cited organizational response to the implementation of an ERP system by the organizations in the reviewed case studies. The linkage between job design and technology is a critical component to understanding the impact of the change on the organization. Although the majority of the cases changed operational processes to fit the system processing, not all of them concentrated on individual job definitions. The organizations that recognized the linkage and proactively created new roles, re-designed jobs, and re-trained workers to accept the changes were more successful in the system implementation and ultimately in behavior modification. The focus on the individual employee response also seems to be a critical component of the acceptance of a new job design. The cited failure cases found an increase in distrust and confusion when the needs of individuals were ignored. In the case of Nestle USA, the system could only be a success after engaging all the workers.

Standardization of data and business processes to support the new system is the second most cited activity within the case studies. Most ERP systems demand that all data conform to a single definition set that can be used in all areas of the business. Each piece of data is then linked through relationships with other pieces of corresponding data. The linkages reinforce the definition and integrity of the data, eliminating data duplication and inaccuracies.

Only two of the cases changed organizational structure in the form of hierarchy or reporting relationships as a response to the system implementation. Owens Corning and Avaya approached the organizational reporting as way to enhance the system structure, as 
the new hierarchies were modeled from the new processes and roles that the system introduced.

\section{Organizational Behaviors}

The two most cited organizational responses to the system are increased coordination and increased operational knowledge in workers. Coordination may be a direct response to the system integration, since organizational departments that before may have been only connected by formal communication or coordinating methods, such as committees, now can rely on a mechanism to assist in the necessary connections. In addition, organizations that lacked formal methods of coordination may for the first time have gained an advantage of coordinating work. Coordination is an expected organizational response of the system characteristic integration, however, not all cases revealed the response.

The increase of individual worker knowledge may be an indirect effect of system integration and a direct effect of the formal training or education component of the implementation. Several of the successful transformation cases indicated that the new way to work was not only linked to individuals who use the system, but also to all workers. In these cases, the education element extended beyond system users and embraced the entire organization. A new shared understanding of the operations was created within those organizations, and system integration creates an ongoing mechanism to enforce the new organizational connections. 
Permanent Change

In all but three of the cases was a lasting change achieved in the first attempt to incorporate new behaviors into daily routines. The non-adoption of organizational wide standards seems to be a factor with failed organizational change. The rejection of a common or shared definition from the organization may indicate a lack of trust in other departments' usage. It also may indicate that the need for common usage is not well understood and the act of consensus is viewed as a relinquishing authority or power. Without consensus, other supporting organizational behaviors, such as communication and coordination, may be impaired. The only case study that was able to reverse the direction of a failing behavior change cited increased communication efforts as the main reason for the reversal. 


\begin{tabular}{|c|c|c|c|}
\hline & \multicolumn{3}{|c|}{ Organizational Response } \\
\hline & Structure & Behaviors & Permanent Change \\
\hline Rolls Royce & $\begin{array}{l}\text { Changed job design; } \\
\text { Created new roles; } \\
\text { Assessed new skills; } \\
\text { Trained all employees }\end{array}$ & $\begin{array}{l}\text { Increased trust; } \\
\text { Increased customer confidence; } \\
\text { Created operational transparency }\end{array}$ & Yes \\
\hline Avaya & $\begin{array}{l}\text { Changed job design; } \\
\text { Changed organizational reporting } \\
\text { structure; } \\
\text { Created global business and } \\
\text { people processes }\end{array}$ & $\begin{array}{l}\text { Increased flexibility; } \\
\text { Increased responsiveness; } \\
\text { Standardized work outputs }\end{array}$ & Yes \\
\hline Owens Corning & $\begin{array}{l}\text { Changed job design; } \\
\text { Created new roles; } \\
\text { Created new processes; } \\
\text { Changed organizational reporting } \\
\text { structure; } \\
\text { Standardized data structures }\end{array}$ & $\begin{array}{l}\text { Centralized coordination; } \\
\text { Dispersed decision-making; } \\
\text { Standardized work output } \\
\end{array}$ & Yes \\
\hline Texas Instrument & $\begin{array}{l}\text { Created new processes; } \\
\text { Standardized data structures }\end{array}$ & $\begin{array}{l}\text { Increased coordination; } \\
\text { Increased performance monitoring }\end{array}$ & Yes \\
\hline Kodak & $\begin{array}{l}\text { Changed Job Design; } \\
\text { Created new roles; } \\
\text { Trained all employees; } \\
\text { Provided support tools }\end{array}$ & $\begin{array}{l}\text { Increased coordination; } \\
\text { Increased internal integration }\end{array}$ & Yes \\
\hline Viskase & $\begin{array}{l}\text { Created new processes; } \\
\text { Standardized data structures }\end{array}$ & $\begin{array}{l}\text { Executed faster decision making; } \\
\text { Increased knowledge of operations } \\
\text { in workforce }\end{array}$ & Yes \\
\hline Diebold & $\begin{array}{l}\text { Created new processes; } \\
\text { Standardized data structures; }\end{array}$ & $\begin{array}{l}\text { Increased workload; } \\
\text { Increased coordination; } \\
\text { Increased communication; } \\
\text { Increased worker knowledge of } \\
\text { operations }\end{array}$ & Yes \\
\hline Nestlé USA & $\begin{array}{l}\text { Created new processes; } \\
\text { Standardized data structures; }\end{array}$ & $\begin{array}{l}\text { Acknowledged the involvement of } \\
\text { the worker; } \\
\text { Increased worker knowledge of } \\
\text { operations }\end{array}$ & $\begin{array}{l}\text { Not at first; Yes upon } \\
\text { completion }\end{array}$ \\
\hline Soft Drink Bottler & $\begin{array}{l}\text { Did not adopt standard processes } \\
\text { and data }\end{array}$ & $\begin{array}{l}\text { Increased frustration; } \\
\text { Created "loss of control" sense }\end{array}$ & Limited \\
\hline $\begin{array}{c}\text { Major } \\
\text { Manufacturer }\end{array}$ & $\begin{array}{l}\text { Did not adopt standard processes } \\
\text { and data; } \\
\text { Did not create an understanding } \\
\text { of the system impact }\end{array}$ & $\begin{array}{l}\text { Created confusion; } \\
\text { Decreased communication; } \\
\text { Increased distrust }\end{array}$ & No \\
\hline
\end{tabular}

Table 1 - Organizational responses by case study 
Summary of Literature Review

The literature review examined information systems research using such social science concepts as organizational change and culture to explain the impact, use, and management of information technology.

Organizational structures shape and reinforce the operating culture by influencing the behaviors that members come to believe are necessary and appropriate. The connection between culture and supporting organizational mechanisms lacks clarity and an in-depth understanding of causality; although the "carriers" or "forces" have been identified in theory, the relationship has yet to be articulated in terms the organizational manager can understand. One structural element that has a diverse and controversial history of exploration is technology. The information systems literature does not clearly link the effect of information systems usage on organizational culture, although if it is studied, the linkage may be predictable and able to be explained.

In the review of organizations using ERP systems as a supporting mechanism of change, several common themes emerge. First, organizations adjust structural elements, such as job design and reporting structures, to better support the system's functionality. Instead of modifying the information system, the other structural elements are changed to accommodate the new process incorporating the information system. Second, organizations respond to the new system characteristics in different manners. For example, the influence of integration may lead to an increase of coordination or act as a mechanism to increase shared understandings. Third, consensus behavior seems to promote successful change. This is evident in the failed case studies. Overall, the 
responses to the system characteristics are visible and do contribute to the lasting changes.

The literature findings imply a linkage between organization structure, information systems, and organizational behavior. Although multiple studies find similar resulting behaviors as a component of technology change, information system researchers have failed to address the conclusion and look for causality. As stated before, a knowledge gap exists with the influences and impacts of specific technology in a particular work setting. It is necessary to understand if and how the technology changes work environments and individual worker perception, to better anticipate and manage organizational outcomes.

In the next section, a conceptual model of research to explore the linkages is presented. 


\section{Conceptual Model}

Figure 1 illustrates the research model for this study. The model consists of three broad components over two time periods - information systems, job design, and organizational culture - which synthesize findings from the literature on technology's direct impact on job design and indirect impact on culture as seen in norms and behaviors in organizations. Although there has been relatively little research on the setting within which information systems and organizational elements are linked, this study tentatively proposes that a change in technology might lead to more integrative behaviors between organizational structure and culture. Designed as a quasi-experimental field study, the research is set during two time periods for one organization. The first time period (referred to as the legacy system or T1) is in a stable organization structural setting in which the three elements of the conceptual model have a tenured existence. The second time period (referred to as ERP system or T2) is after the structural element of information systems has been entirely replaced. The comparison of the settings extends the understanding of how organizational structure mechanisms, in the form of information systems, can directly impact the individual and organizational perceptions' of job design characteristics and organizational operating culture dimensions and the relationship between information technology, job design, and culture. The conceptual model builds on previous work exploring the connection between job characteristics and organizational culture (Cooke and Szumal, 2000), by extending the model to include information systems environment characteristics. Each of the three model components is discussed next. 


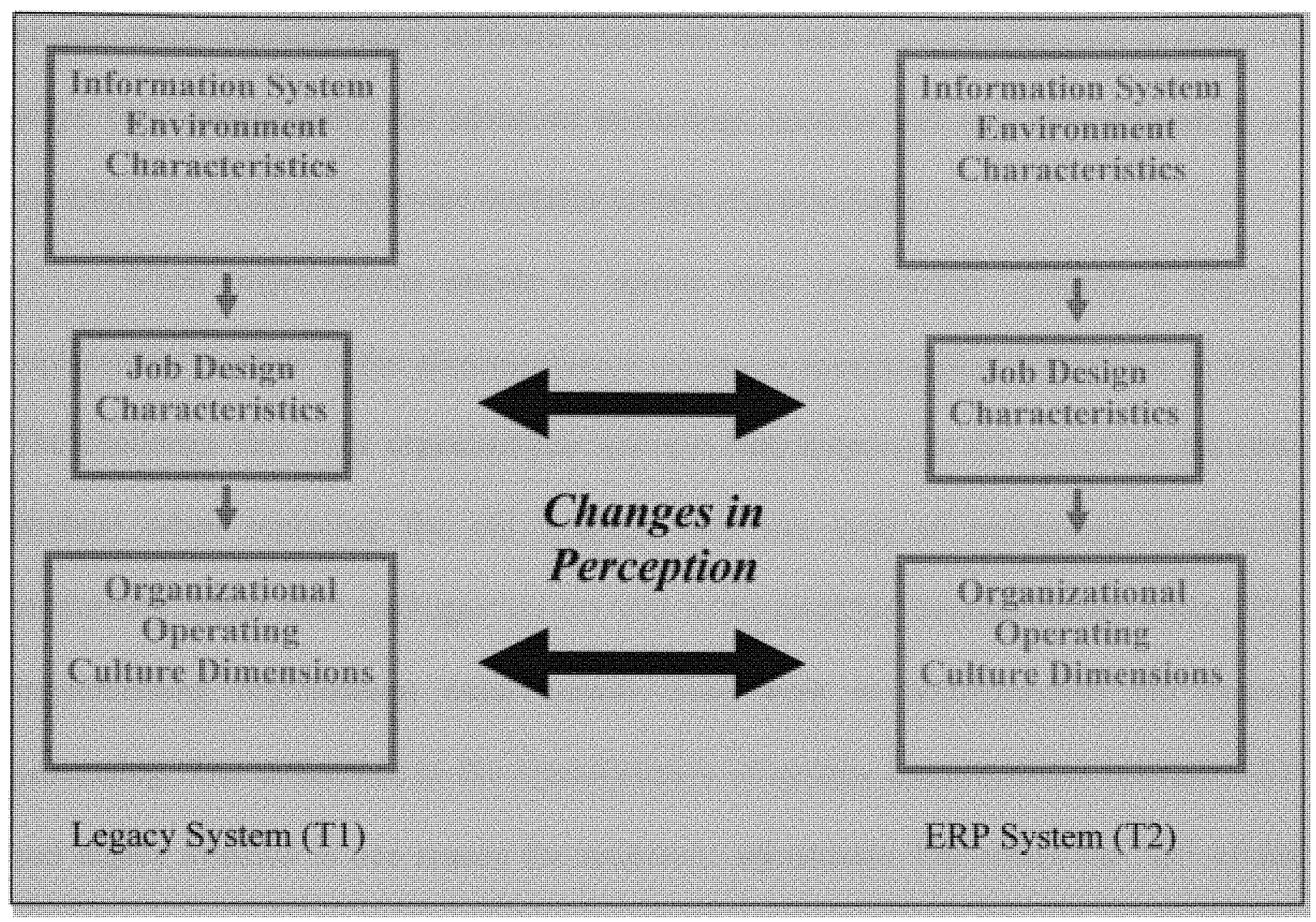

Figure 1 - Conceptual Model

Information Systems

As mentioned in the previous section, information systems are very diverse and contain a very wide range of functions and characteristics. Most large modern organizations, however, always use information systems for administrative purposes. Due to the continuing and unchanging nature of these administrative functions, information systems supporting the work are seldom replaced. Organizations may continue to use older antiquated technologies to save the expense and effort of moving to new applications. Systems with this type of longevity are referred to as legacy systems. System characteristics are common performance and presentation attributes that describe how the system processes and interacts with users. Although all information systems 
contain these characteristics, software represents and uses the characteristics in different ways. New functionality and advanced technology features will also vary depending on the extent to which the software contains certain characteristics.

When organizations decide to update their information systems, a popular replacement product is the aforementioned enterprise resource planning system or ERP. The challenge that most organizations face (as demonstrated in the review of the case studies) when replacing the old systems with this type of technology is one of conformity. Most ERP packages are not able to be modified beyond certain limits. The implementing organization must modify their work to accommodate the system processing. The difference in the representation of system characteristics between ERP and legacy systems is one reason for necessary adjustment. The closer the "fit" of the system characteristics to the task and job, the less change will be required for the worker. It is in the adjusting process of the job design and resulting behaviors that this study is focused. For this study, information systems, both the legacy and ERP systems, will be evaluated based on characteristics that support the work task in the job.

Job Design

In examining technology in organizational work activities, many studies have found the greatest impact is on job design (Attwell and Rule, 1984; Argyres, 1999; Cooke and Szumal, 2000). Job design or job characteristics theory is a behavioral approach that focuses on the objective characteristics of an employee's job and links their influence to that said employee (Turner and Lawrence, 1965). A number of studies have addressed the importance of individual differences in responses to job characteristics (Pierce and 
have addressed the importance of individual differences in responses to job characteristics (Pierce and Dunham, 1976). However, it should be noted that the explanation for difference in reaction between workers has not be clearly identified (Stone, 1976; Stone, Mowday, et al., 1977).

The most complete and best known theory for explaining worker responses to job characteristics is that presented by Hackman and Oldman (1974). According to their theory, any job can be described in terms of five core job characteristics: skill variety, task identity, autonomy, task feedback, and task significance. These core dimensions are said to influence three critical psychological states of workers: the experienced meaningfulness of work, the experienced responsibility for outcomes of work, and the knowledge of actual results of work activities. High levels of the critical psychological states will lead to favorable personal and work outcomes. Hackman and Oldman's research and subsequent work has supported the link between job dimensions and personal and work outcomes (Dunham, Pierce, et al., 1983).

Linking organizational structure, in the form of information systems, to job characteristics, this study examines the influence and connections in the resulting worker perception of the job design. The first set of research questions to be formulated for this study is the following:

How do the perceived legacy system characteristics influence an individual's perceived job design characteristics?

How do the perceived ERP system characteristics influence an individual's perceived job design characteristics?

When comparing the legacy time period and the ERP time period, how are the perceived job design characteristics and their linkages with information system characteristics different? 
Culture

The last component of the conceptual model is part of the organizational structure - culture. Culture is a complex factor and notoriously difficult concept to define. A earlier sociological definition of culture is that it is the shared set of beliefs that influence what is considered meaningful and valuable (Weber, 1949). Clarke et al. (1981) broaden the definition of culture to argue that it is constituted and expressed through institutions, social relations, customs, materials, objects, and organizations. The word "culture" is typically associated with societies and nations, or relating to ethnic or regional groups. However, it is also applied to other human groups such as organizations, professions, or families.

Geert Hofstede, an early researcher in work-related values among different nationalities, defines culture as "the collective programming of the mind which distinguishes the members of one human group from another" (Hofstede, 1980, 21). It is the grouping of individuals in a work setting that is the level of analysis for the organizational scientist. The theme of shared understanding influences a more current definition of organizational culture as the set of important assumptions (often unstated) that members of communities share in common (Sathe, 1985).

The use of the concept of culture in this research is to represent the "collective programming" of a group to exhibit certain types of behavior styles. The benefit of this definition is the ability to view culture as simply a static product of the "program." Complex unseen forces mold ideas and feelings. These are cultural motivations and can be seen in the operating behaviors. 
Derived from a 1940's study of field theory by Lewin (1951), the first term to reference the quantitative study of attitudes within organizations was "organizational climate." His work to represent any particular social process as part of a larger context or field was closely linked to the context references of the Gestalt psychology of individual perception (Ashkanasy, Broadfoot, et al., 2000). Climate was a way to characterize the context of a group setting in a simple manner, describing the attitudes, feelings and social processes that occurred in groups. Rensis Likert (creator of the Likert scale for measuring attitudes) had profound effects on organizational climate research. He studied climates of organizations in order to improve performance (Likert, 1961), and the scale he invented provided a starting point to capture quantitatively an organization's climate. The use of the term "climate" in organizational studies became a reference for a quantitative, nomothetic, or comparative census of a group's perceptions (Denison, 1996). It is useful for this study that the original way to distinguish between perceptions of attitude is still being used in the modern measurement of organizational culture. This measurement method accurately captures the attitudes of participants.

Common usage by organizational researchers of the terms "climate" and "culture" reflect different views on ontology, epistemology, and methods. Constituting the distinction between organizational culture research and organizational climate research, they are overlapping interpretations of the same phenomenon (Denison, 1996).

The concept of culture was infused in 1980s general management research by organizational scientists (Ouchi, 1980; Peters and Waterman, 1982; Deale and Kennedy, 1982), who constructed the concept of culture to be a single variable or characteristic of an organization. They argued that a "strong culture" distinguishes successful 
organizations from other organizations (Peters and Waterman, 1982). In their view, a "strong culture" facilitates coordination in communication, thus giving strong cultured companies a competitive advantage (Ouchi, 1980; Weick, 1985). In empirical studies, a strong culture seemed to show a positive correlation with short-term performance rather than a lasting competitive advantage trait (Denison, 1990; Gordon and DiTomaso, 1992). Akin and Hopelain (1986), Graves (1986), Kilmann and Covin (1988), and Sathe (1985) employed this one-dimensional notion of culture to judge and characterize organizational productivity and efficiency.

Culture became a strategic tool to sharpen organizational performance in the works of Fombaun (1983) and Scholz (1987), and a mechanism for control by Kunda (1992) and Barley (1988). These studies use culture as a manipulative device that can impact an individual's behavior within a group setting. This is an important finding that will be used in this research: organizational culture can be changed and used to control organizational settings.

According to Schein's (1985) three-level pyramid typology, organizational culture is derived from the base representing underlying pattern of members' assumptions shared as a result of common experiences in their working life. These assumptions are reflected in, and give meaning to, values which support observable artifacts, and patterns of behavior. He breaks his methodology into three levels:

- Artifacts and Creations - the most visible manifestations of organizational culture. Includes physical space, technology, art, symbols, language, mottos and overt behavior. This layer is the visible system or organizing interpersonal relationships, status levels, gender, age, and other highly visible characteristics. 
- Values and Ideology - the rules, principles, demands, values, morals, and ethics that guide society and the means by which to accomplish them. These are the espoused expressions by individuals as the organization faces and deals with new situations.

- Basic Assumptions and Premises - the underlying "unconscious" thoughts and behavior around human nature. These define the core or essence for deciphering the values, artifacts, and trade patterns that characterize cultural phenomena.

Organizational members use these assumptions to guide their behavior and attitudes (Schein, 1981) (a reproduction of his organizational culture model appears in Figure 2).

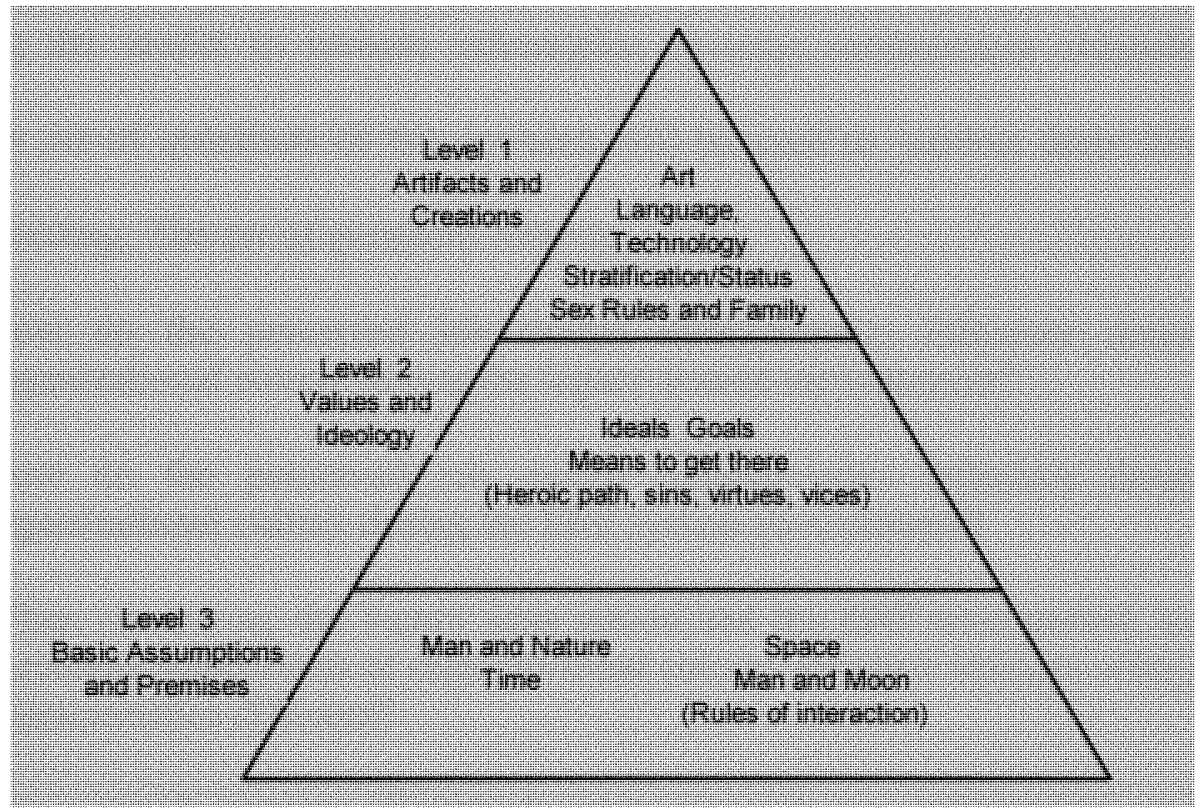

Figure 2-Schein's model of organizational culture

Schein's detailed conceptualization of organizational culture supports the examination of outward artifacts and espoused ideas as expressions of organizational culture. Examination of organizational culture can be applied through the appropriate level and supported by the layer beneath. An examination of organizational artifacts may 
lead to an understanding of the values and ideology used to create and use them, and Level 1 will be used as a base of study in this research.

Cooke and Szumal (2000) use the behavioral definition of culture to investigate how antecedent variables, such as job characteristics, are related to cultural behaviors. They find a linkage between job design and different groupings of organizational behavior. Their results demonstrate how different organizational structures shape the operating culture by influencing the behaviors members come to believe are necessary and appropriate in the setting. The conceptual model builds upon this previous work by extending the model to include information systems environment characteristics. A secondary set of questions of this research will be used to validate these findings in a different setting. The set of questions follows:

How do the perceived job design characteristics influence dimensions of organizational culture during the legacy system environment?

How do the perceived job design characteristics influence dimensions of organizational culture during the ERP system environment?

When comparing the legacy time period and the ERP time period, how are the perceived dimensions of organizational culture and their linkages with job design characteristics different?

The second chapter discussed prior research on information systems and presented a case study analysis of ERP in organizations. Several major factors were identified as having relevance to the information systems being used as a supportive element of organizations. The first is that information systems influence organizational operating environment behavior through the shaping and enforcement of organizational structure. Second, information system environment characteristics such as integration or standardization are used differently based on the organizational setting. Next, the 
influence of the information system characteristics in the organizational structure can be seen in the design and perception of work tasks and work processes. The system supports designed behavior through each of the work processes and therefore can be seen as an enforcer of the operations. The organizational operating culture or environment is composed of the daily routines exhibited in the form of behaviors referred to as organizational operating culture dimensions. This argument forms the basis of elements included in the conceptual model (see Figure 1).

In the next several sections, the research design of the study will be discussed. The components of the conceptual model link structural influences to the organizational operating culture. The components' definition used in this research and the manner to collect the information regarding each component is discussed in the subsequent sections of research design and research method. 
IV. Research Design and Methodology

According to Weick (1985), when conducting research in the social sciences there is a major theoretical assumption a researcher must make regarding the subject of analysis: subjects are basically alike or they are unique. Given the assumption one makes, one may study a topic via a selective examination of many subjects, or an intensive examination of a few. In this research project, it is not known whether the relationship between transforming organizations' culture dimensions and system characteristics are essentially alike or if they are unique. If relationships are alike, then an examination of many should reveal consistent patterns between the dependent and independent variables. If such patterns can be identified, then researchers and managers will have a better understanding of the interaction of relevant components. This would enable more complex theories and testable hypotheses regarding the components' interactions. The hypotheses put forth in the previous chapters are based on the assumption that these relationships are basically alike, and thus some consistent pattern of association can be found between the independent and dependent variables.

Exploratory field research is appropriate when little is known about a subject. The way to select the method of research depends on three conditions: the type of research question, the control an investigator has over actual behavioral events, and the focus on contemporary as opposed to historical phenomena (Yin, 1994). Case study research or surveys supports the examination of the phenomena. Survey research was chosen to be used in this project to assist in identifying patterns in the relationship that can be statistically supported. Several participants were interviewed informally to validate the empirical findings. A basic connection between system characteristics, job 
characteristics, and operating culture must first be found and then supported before further research is able to examine the connection more closely. This research is the first attempt to view the technology as an independent variable influencing organizational culture through job design. After a more detailed understanding of the connections is understood, other methods may be used to produce a richer understanding.

Questionnaires

Elements from three questionnaires were used to collect data on the variables described in the research model. A review of instruments employed in user assessments of technology, job design assessment, and organizational culture analysis was undertaken to find the instruments that looked for responses based on behavior and work task rather than attitude of the work setting or information system. The result was the inclusion of three instruments: the Task-Technology Fit survey (Goodhue, 1995), the Job Diagnostic Survey instrument (Hackman and Oldham, 1974), and the Organizational Culture Inventory $(\mathrm{OCI})^{4}$. The following section will describe, review the validity and reliability of each instrument, and detail each of the items used to measure the variables.

\section{Information System Characteristics}

System characteristics supporting work tasks will be used to describe information systems, the first factor of the conceptual model. The system assessment used in this research seeks an evaluation of how well the technology fits the work tasks performed by the organizational member. Exploring organizational routines will provide a behavioral response to the influence of the technology rather than an emotional or attitudinal

\footnotetext{
${ }^{4}$ Organizational Culture Inventory $(1989$ by Human Synergistics, Inc.
} 
response. The Task-Technology Fit model $^{5}$ is based on users evaluating the extent to which the system meets task needs and their individual abilities (Goodhue, 1998). The model's questionnaire defines twelve constructs: the data detail, data accuracy, data compatibility, data location, data accessibility, data meaning, data concurrency, assistance, ease of use, reliability, presentation, and confusion or difficulty in understanding the system or the computer interface.

User assessments typically explore certain qualities of information systems in a continuum from negative to positive (Melone, 1990). Twenty-three questions using a seven-point scale from strong agreement to strong disagreement with the statement evaluate the twelve constructs.

Seven constructs relate directly to users identifying the data they need to evaluate information systems. Data detail is the right level of data in the delivered format for the purposes used in the job. The data detail construct is how it is summarized or factored to properly fit the necessary use in the job tasks (O'Reilly, 1982). Data accuracy refers to the correctness of interpretation for use in the task (Zmud, 1978). Data compatibility is the ease with which data from different sources can be aggregated or compared without inconsistencies, as data that comes from several different sources may be incompatible (Epstein and King, 1982; Bailey and Pearson, 1983). The user must decide if the data is reliable enough to be used for the task at hand. Data location refers to the ability to locate the needed data assuming that the user has the correct authority to use the data, and data accessibility refers to whether the proper authority has been assigned to users who need

\footnotetext{
${ }^{5}$ Model of Task-Technology Fit and User Evaluations can be found in Goodhue, D. L. (1995). "Understanding User Evaluations of Information Systems." Management Science 41(12): 1827.
} 
to use data in a task (Bailey and Pearson, 1983). Data meaning refers to how clear the meaning is in the representation of the data and if it is understood by the user (Epstein and King, 1982). Concurrency of data refers to how timely the information is to assist the task (Zmud, 1978). Each of these constructs demonstrates a different characteristic of the data used in the task.

Five constructs assess the usage support of the information system. Assistance refers to the ability to gain help or information about system elements and the data. It may come in the form of system information or from personnel supporting the system's use (Swanson, 1987). Ease of use refers to the ease of doing what the user wants to do using the system. If it is easy to accomplish a task using the system, then ease of use will be high; the opposite will be true if the system is perceived as difficult. Reliability refers to if the system is operational when the user needs to use it for the task (Bailey and Pearson, 1983). Presentation refers to the display of data on the screen and in reports and how easy the presentation of the data is to interpret (Zmud, 1978). Confusion caused by the system is the result of poor organization or a lack of understanding of the system by the user doing the task.

The Task-Technology Fit survey is a unique survey in the information systems field. It combines the attribute of the technology with the behavior necessary to support the task. Other investigations have studied the impact of users' beliefs and attitudes on their usage behavior and many of the models incorporate perceived ease of use as a determinant of acceptance, such as the Technology Acceptance Model (TAM) (Davis, 1989; Davis, 1993). TAM suggests that two specific beliefs - perceived ease of use and perceived usefulness - determine one's behavioral intention to use a technology (Taylor 
and Todd, 1995). Although other user assessment surveys may also try to assess the technology for usage, the linkage between the job design and the system characteristics is not examined in them. The behavior focus of the Task-Technology Fit survey (Goodhue, 1995 ) is essential to understand how the system changes the job design and work. It was selected due to its connection to task behavior and because the questionnaire has acceptable levels of reliability and validity. (Table 2 contains reliability scores by construct).

The Task-Technology Fit instrument was designed with at least two parallel questions for each construct, and the questions are randomly ordered. Internal consistency of each construct is reflected in Cronbach's Alpha ${ }^{6}$ score listed by construct in Table 2. The discriminate validity of the instrument is demonstrated by a confirmatory factor analysis presented in Goodhue (1995). The results show the chi-squared value is significant for the twelve separate dimensions at the .001 level.

Two separate questions are asked about each of the twelve constructs. An additional item was asked in the data compatibility construct. The question asks for the respondent to indicate the extent to which they agree or disagree with the statements on a 7 point Likert scale of $1=$ strongly disagree, $4=$ neither agree nor disagree, and $7=$ strongly agree. By construct, the questions are the following:

Data Detail items are:

1. Sufficiently detailed data is maintained by the university.

2. The university maintains data at an appropriate level of detail for my purposes.

Data Accuracy items are:

\footnotetext{
${ }^{6}$ Cronbach's Alpha is a coefficient of reliability and not a statistical test. It measures how well a set of items (or variables) measures a single unidimensional latent construct.
} 
3. The data that I use or would like to use is accurate enough for my purposes.

4. There are accuracy problems in the data I use or need.

Data Compatibility items are:

5. When it's necessary to compare or aggregate data from two or more different sources there may be unexpected or difficult inconsistencies.

6. There are times when supposedly equivalent data from two different sources is inconsistent.

7. Sometimes it is difficult or impossible to compare or aggregate data from two different sources because the data is defined differently.

Data Location items are:

8. It is easy to locate university data on a particular issue, even if I haven't used that data before.

9. It is easy to find out what data the university maintains on a given subject.

Data Accessibility items are:

10 . I can get data quickly and easily when I need it.

11. It is easy to get access to data that I need.

Data Meaning items are:

12. On the reports or systems I deal with, the exact meaning of data elements is either obvious or easy to find out.

13. The exact definition of data fields relating to my task is easy to find out.

System Assistance items are:

14. I am getting the help I need in accessing and understanding the data.

15. It is easy to get assistance when I am having trouble finding or using data.

Ease of Use of Hardware and Software items are:

16. It is easy to learn how to use the computer systems that give me access to data.

17. The computer systems that give me access to data are convenient and easy to use.

System Reliability items are:

18. The data is subject to frequent systems problems and "downages."

19. I can count on the system to be "up" and available when I need it.

Currency items are:

20. I can't get data that is current enough to meet my needs.

21. The data is up-to-date enough for my purposes.

Presentation items are:

22. The data that I need is displayed in a readable and understandable form. 
23. The data is presented in a readable and useful format.

Confusion items are:

24. There are so many different systems or files, each with slightly different data, that it is hard to understand which one to use in a given situation.

25. The data is stored in so many different places and so many forms, it is hard to know how to use it effectively.

\begin{tabular}{|cc|cc|}
\hline $\begin{array}{c}\text { Task- } \\
\text { Technology Fit }\end{array}$ & $\begin{array}{c}\text { Cronbach's } \\
\text { Alpha }\end{array}$ & JDS & $\begin{array}{c}\text { Cronbach's } \\
\text { Alpha }\end{array}$ \\
\hline The level of & & & \\
detail & 0.85 & Variety & $.65-.78$ \\
Accuracy & 0.83 & Identity & $.74-.83$ \\
Compatibility & 0.82 & Significance & $.72-.83$ \\
Location & 0.77 & Autonomy & $.68-.77$ \\
Accessibility & 0.84 & Feedback & $.65-.81$ \\
& & Work with & \\
Meaning & 0.78 & Others & $.65-.83$ \\
Assistance & 0.87 & & \\
Ease of Use & 0.77 & & \\
System & & & \\
Reliability & 0.77 & & \\
Currency & 0.73 & & \\
Presentation & 0.86 & & \\
Confusion & 0.73 & & \\
\hline
\end{tabular}

Table 2 - Cronbach's Alpha by TTF and JDS constructs

Job Design Characteristics Instrument

The well-known instrument, the Job Diagnostic Survey (JDS) instrument (Hackman and Oldham, 1974), was selected to measure job design characteristics. This survey has been used in twenty documented studies (Fields, 2002) and has been revised by Idaszak and Drasgow (1987) for wording. The five job characteristics measured by the instrument are: skill variety, task identity, autonomy, task feedback, and task significance. The supplemental related JDS construct of "dealing with others" or "work 
with others" is the degree the job requires employees to work closely with other people in carrying out the work activities. This additional factor has been added to the survey and is used in this study.

Job variety is the degree to which the job requires many different skills and talents of the worker. It measures the non-routineness of the job. If a task requires workers to engage in activities that challenge or stretch their skills, they almost invariably experience that task as meaningful (Hackman and Oldham, 1980). Variety is influential in the meaningfulness of the job. Another meaningful variable is job or task identity. This is the extent to which the job encompasses either an entire piece of work or makes only a small contribution to the larger work effort. When workers have an entire task, they tend to see that task as more meaningful. Organizational members care about their work more when they are doing a whole job rather than a piece of the job (Hackman and Oldham, 1980). Job autonomy as defined by the creators is the "degree to which the job provides substantial freedom, independence and discretion of the employee in scheduling the work and in determining the procedures to be used in carrying it out" (Hackman and Oldham, 1974). Autonomy fosters a feeling of personal responsibility for the work outcomes. As autonomy increases, workers tend to feel more personal responsibility for job successes and failures. Job feedback is the extent that the job itself provides information about the effectiveness of the worker's performance; feedback contributes to the knowledge of the direct results of the job contribution. Task significance is to what degree the job affects the broader scheme of things. It is the amount of impact that the job has on the lives of other people, whether in or outside the organization. The "work with others" construct describes if the job requires joint activities with others or if the job can be completed 
without other worker involvement. For this study, job design will be defined by the six characteristics.

The instrument's variables were found to be empirically distinct in structural equation models by Renn and Vandenberg (1995). Reliability is inferred from coefficient alpha values for skill variety ranging from .65 to .78 . Alpha values ranged from .74 to .83 for task identity, from .72 to .83 for task significance, from .68 to .77 for autonomy, and from .65 to .81 for feedback (Munz, Huelsman et al., 1996; Renn and Vandenberg, 1995; Siegall and McDonald, 1995; Spector, Jex et al., 1995; Steel and Rentsch, 1997; Taber and Taylor, 1990). The test-retest reliability of the JDS was r-.62 (Taber and Taylor, 1990).

Skill Variety items ask the respondents to circle the number that most accurately describes their jobs. The anchors are a 7 level range with $1=$ very little: The job requires me to do the same routine things over and over again; $4=$ moderate variety; $7=$ very much: The job requires me to do many different things using a number of different skills and talents. The item is:

1. How much variety is there in your job? That is, to what extent does the job require you to do many different things at work, using a variety of your skills and talents?

(The response scale for the next two items is $1=$ very inaccurate, 2 = mostly inaccurate, 3 = slightly inaccurate, $4=$ uncertain, $5=$ slightly accurate, $6=$ mostly accurate, and $7=$ very accurate.)

2. The job requires me to use a number of complex or high-level skills.

3. The job is quite simple and repetitive. (This item is reversed scored.)

Task Identity item anchors are a 7 level range with $1=$ My job is only a tiny part of the overall piece of work: The results of my activities cannot be seen in the final 
product or service; $4=$ My job is a moderate-sized "chunk" of the overall piece of work:

My own contribution can be seen in the final outcome; $7=$ My job involves doing the whole piece of work, from start to finish: The results of my activities are easily seen in the final product or service. The items are:

1. To what extent does your job involve doing a "whole" and identifiable piece of work? That is, is the job a complete piece of work that has an obvious beginning and end? Or is it only a small part of the overall piece of work, which is finished by other people or by automatic machines?

(The response scale for the next two items is $1=$ very inaccurate, $2=$ mostly inaccurate, 3 = slightly inaccurate, $4=$ uncertain, $5=$ slightly accurate, $6=$ mostly accurate, and $7=$ very accurate.)

2. The job provides me the chance to completely finish the pieces of work I begin.

3. The job is arranged so that I can do an entire piece of work from beginning to end. (This item is revised from Idaszak and Drasgow's [1987] study.)

Task significance item anchors are a 7 level range with $1=$ not very significant:

The outcomes of my work are not likely to have important effects on other people; $4=$ moderately significant; $7=$ highly significant: The outcomes of my work affect other people in very important ways. The items are:

1. In general, how significant or important is your job? That is, are the results of your work likely to significantly affect the lives or well-being of other people?

2. This job is one where a lot of other people can be affected by how well the work gets done.

3. The job itself is very significant and important in the broader scheme of things. (This item is revised from Idaszak and Drasgow's [1987] study.)

Autonomy item anchors are a 7 level range with $1=$ very little: The job gives me almost no personal "say" about how and when the work is done; 4 = moderate autonomy: Many things are standardized and not under my control, but I can make some decisions 
about the work; $7=$ very much: The job gives me almost complete responsibility for deciding how and when the work is done. The items are:

1. How much autonomy is there in your job? That is, to what extent does your job permit you to decide on your own how to go about doing the work?

(The response scale for the next two items is 1 = very inaccurate, 2 = mostly inaccurate, 3 = slightly inaccurate, $4=$ uncertain, $5=$ slightly accurate, $6=$ mostly accurate, and $7=$ very accurate.)

2. The job gives me considerable opportunity for independence and freedom in how I do the work.

3. The job denies me a chance to use my personal initiative or judgment in carrying out the work. (This item is revised from Idaszak and Drasgow's [1987] study.)

Feedback item anchors are a 7 level range with $1=$ very little: The job itself is set up so I could work forever without finding out how well I am doing; $4=$ moderately: Sometimes doing the job provides "feedback" to me, sometimes it does not; $7=$ very much: The job is set up so that I get almost constant "feedback" as I work about how well I am doing.

1. To what extent does doing the job itself provide you with information about your work performance? That is, does the actual work itself provide clues about how well you are doing, aside from any "feedback" co-workers or supervisors may provide?

(The response scale for the next two items is 1 = very inaccurate, 2 = mostly inaccurate, 3 = slightly inaccurate, $4=$ uncertain, $5=$ slightly accurate, $6=$ mostly accurate, and $7=$ very accurate.)

2. Just doing the work required by the job provides many chances for me to figure out how well I am doing.

3. After I finish a job, I know whether I performed well.

Work with others item anchors are a 7 level range with 1 = very little: dealing with people is not at all necessary in doing the job; $4=$ moderately: some dealing with others is necessary; 7 = very much: dealing with other people is an absolutely essential and crucial part of doing the job. 
2. To what extent does your job require you to work closely with other people (either clients or people in related jobs in your own organization?

(The response scale for the next two items is 1 = very inaccurate, $2=$ mostly inaccurate, 3 = slightly inaccurate, $4=$ uncertain, $5=$ slightly accurate, $6=$ mostly accurate, and $7=$ very accurate.)

2. The job requires a lot of cooperative work with other people.

3. The job can be done adequately by a person working alone-without talking or checking with other people.

\section{Culture Instrument}

Different methodological approaches in the study of organizational culture have emerged, with survey tools as the most reported and used. The survey approach measures culture though questionnaires of individuals in the organization (Hofstede, 1980; Kilmann and Covin, 1988). The analytical prescriptive approach empirically studies culture through its manifestation in rituals and stories (Trice and Beyer, 1984), and the ethnographic approach studies culture as it is enacted through observations and interviews with a limited set of informants. Even with the variety of styles, the majority of culture research is still directed toward assessment questionnaires and surveys (Rousseau, 1990). Using Schein's organizational typology, three instruments focus on the first level - patterns of behavior or norms of behavior - within an organizational setting: Allen and Dryer's (Allen and Dyer, 1980) Norm Diagnostic Index (NDI), one developed by Kilmann and Saxton (1983), and the organizational culture profile (or OCP) developed by Cooke and Lafferty (1988). Of the three surveys, the OCP is the only device reported to be reliable and possessing consensual, construct, and criterion validity within its two constructs (Ashkanasy, Broadfoot et al., 2000). The first construct focuses on concern for people and task and measures how organizational members are expected 
to think and behave regarding their job and other organizational members. The second construct - security versus satisfaction - represents the degree to which people are encouraged to avoid conflict and protect themselves versus being innovative and risktaking within the organization (Rousseau, 1990).

Using the OCP, Cooke and Lafferty developed improvements on the ten behavior norm dimensions ${ }^{7}$ to describe the culture. They refined the survey to produce a second instrument - the organizational culture inventory (OCI). This instrument categorized behaviors into more descriptive classifications of group behavior. The OCI measures twelve behavioral norms ${ }^{8}$ associated with three distinctive categories describing an organizational culture: constructive, passive/defensive, and aggressive/defensive. Constructive organizational cultures are characterized by encouraging members to interact with people and approach tasks in ways that will help them meet their higher order satisfaction needs. Passive/defensive organizational cultures encourage or implicitly require members to interact with people in ways that will not threaten their own personal security. Aggressive/defensive organizational cultures encourage or drive members to approach tasks in forceful ways to protect their status and security. The

\footnotetext{
${ }^{7}$ The dimensions are leadership, structure, innovation, job performance, planning, communication, environment, humanistic workplace, development of the individual, and socialization on entry. Cooke, R. A. and J. L. Szumal (1993). "Measuring normative beliefs and shared behavioral expectiations in organizations: The reliability and validity of the Organizational Culture Inventory." Psychological Reports(72): 1299-1330.

${ }^{8}$ The constructive culture norms are achievement, self-actualizing, humanistic-encouraging, affiliate. The passive/defensive culture norms are approval, conventional, attended, and avoidance. The aggressive/defensive cultural norms are opposition, power, competitive, and perfectionist. Cooke, R. A. and J. L. Szumal (2000). Using the Organizational Culture Inventory. Handbook of Organizational Culture and Climate. N. M. Ashkanasy, C. Wilderom and M. F. Peterson. Thousand Oaks, $\mathrm{CA}$, Sage Publications, Inc.
} 
purpose of the OCI instrument is to identify not only consensus behavior but also contradictory norms that may work against the sanctioned culture.

For the purpose of this study, organizational culture will be defined as the behavioral norms that are determined by the organizational conditions and realities that members face on a day-to-day basis and referred to as the organizational operating culture. This is the definition used in the underlying theory of the OCI instrument.

Studies utilizing the OCI have included: to direct, evaluate, and monitor an organization and study of change (Gaucher and Kratochwill, 1993), to enhance system reliability and safety (Haber, O'Brien et al. 1991), and to promote partnership of strategic alliances in a study of mergers and acquisitions (Slowinski, 1992). These and other industry studies (Kotter and Heskett, 1992; Shurber and Haber, 1992) have produced information regarding the ways in which culture operates in different types of organizations and have an understanding of how culture works within an organization.

The connection between organizational culture and the supporting organizational mechanisms that shape it is one area that has not been studied extensively. Although the "carriers" or "forces" have been identified in theory, the relationship has yet to be articulated in terms the organizational manager can understand. One structural element that has a diverse and controversial history of exploration is information systems technology. However, the information systems literature does not clearly show how information systems usage influences organizational culture - a linkage that may be predictable and able to be explained if studied. Culture is a fuzzy, imprecise, and not easily measured phenomena that has a practical connection to technology in organizations (Hoffman and Klepper, 2000). Theorized as a contributor, the link between 
organizations (Hoffman and Klepper, 2000). Theorized as a contributor, the link between information systems and culture has remained an invisible occurrence affecting culture within institutions. This study will make the linkage visible. A closer examination of prior research and theory developments of information system technology and culture is discussed in the next section.

The Organizational Culture Inventory is a quantitative instrument that measures twelve basic subscales which are the following: Humanistic/Helpful, Affiliation, Achievement, Self-Actualization, Approval, Conventionality, Dependence, Avoidance, Oppositional, Power, Competitive, and Perfectionism. These subscales reflect a circular diagram model based on the intersection of two dimensions which are task-people and security-satisfaction and which proved the four secondary subscales of the questionnaire. There are 120 survey items, each one rated on a 1-5 Likert scale. The Cronbach's alpha coefficient of internal reliability has been reported to range from $.67-$ .92 (Cooke and Rousseau, 1988). There have been moderately high levels of withinorganization agreement on the OCI across samples for validity of the measure (Cooke and Rousseau, 1988). In a study that compared four organizational culture instruments for correlation and factor analysis, the OCI instrument had the best internal reliability and its subscales loaded on four of the six dimensions tested (Xenikou and Furnham, 1996). The OCI was selected due to its examination of behavior and task characteristics within the organization and its wide use in the field by commercial researchers and practitioners (Rousseau, 1990). The instrument is a copyrighted product and is used with the permission of the copyright holder in this study. Due to the agreement of the permission, the survey items cannot be reproduced. 
Detailed Hypothesis

The last chapter provided a discussion of literature bases that yielded several factors that might influence the overall impact of information systems in general, and the impact on job design and organization operating culture in particular. A conceptual model summarizing these factors was presented at the end of the chapter (Figure 2). The model is broken into two components to support each major hypothesis, each containing a set of dependent and independent variables that will be representative of the information system environment characteristics and the job design characteristics, and the job design characteristics and organizational operating culture dimensions. This section explicitly identifies and explains the three elements of the conceptual model in terms of individual measurable variables.

\section{Research Question Set \#1}

The first two research questions on page 45 , examine the association between independent information system characteristic variables and dependent job design characteristic variables. The question, how do the perceived system characteristics of information systems influence an individual's perceived job design characteristics, was asked before and after the implementation of an ERP system. Figure 3 is a detailed representation of the variables. From the previously discussed rationale, it is possible to formulate the following detailed hypotheses. Representation of each time period in designated by $a$ for the legacy environment and $b$ for the ERP environment. 
Data detail is the right level of data in the delivered format for the purposes used in the job. If the information is perceived to have the correct level of detail to support a task, then a positive relationship between data detail and the job design characteristics should be found.

H1a,b: The perception of the information system's data detail will be positively associated with the worker's perception of job variety in the: (a) legacy environment; (b) ERP environment.

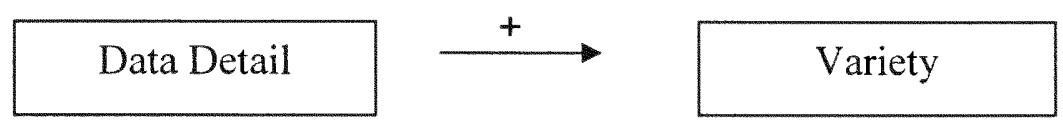

H2a,b: The perception of the information system's data detail will be positively associated with the worker's perception of job identity in the: (a) legacy environment; (b) ERP environment.

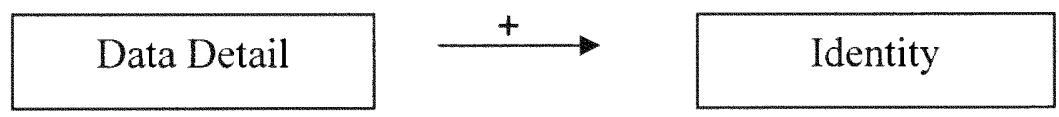

H3a,b: The perception of the information system's data detail will be positively associated with the worker's perception of autonomy in the: (a) legacy environment; (b) ERP environment.

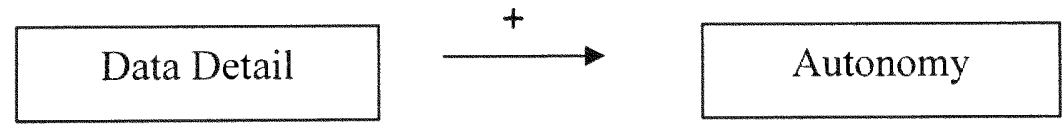

H4a,b: The perception of the information system's data detail will be positively associated with the worker's perception of feedback in the: (a) legacy environment; (b) ERP environment.

Data Detail

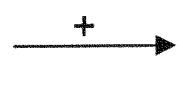

Feedback 
H5a,b: The perception of the information system's data detail will be positively associated with the worker's perception of significance in the: (a) legacy environment; (b) ERP environment.

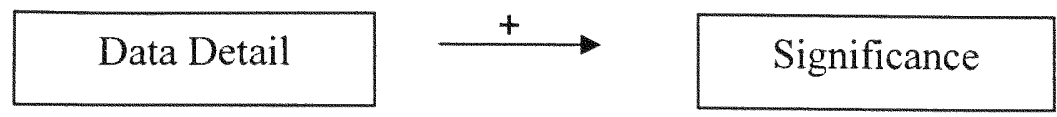

H6a,b: The perception of the information system's data detail will be positively associated with the worker's perception of working with others in the: (a) legacy environment; (b) ERP environment.
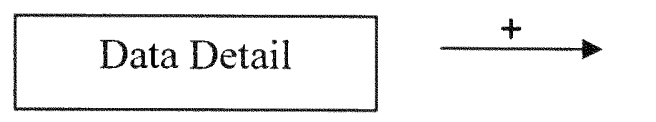

\section{Work with Others}

Data Accuracy

Data accuracy is the right level of data in the delivered format for the purposes used in the job. If the information is perceived to be accurate to support a task, then a positive relationship between data accuracy and the job design characteristics should be found.

H7a,b: The perception of the information system's data accuracy will be positively associated with the worker's perception of job variety in the: (a) legacy environment; (b) ERP environment.

\section{Data Accuracy}

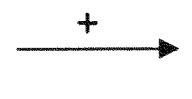

Variety

H8a,b: The perception of the information system's data accuracy will be positively associated with the worker's perception of job identity in the: (a) legacy environment; (b) ERP environment.

Data Accuracy

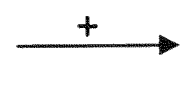

\section{Identity}


H9a,b: The perception of the information system's data accuracy will be positively associated with the worker's perception of autonomy in the: (a) legacy environment; (b) ERP environment.

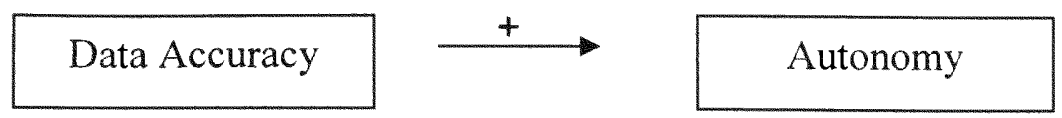

H10a,b: The perception of the information system's data accuracy will be positively associated with the worker's perception of feedback in the: (a) legacy environment; (b) ERP environment.

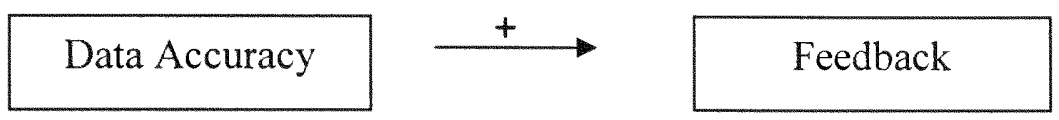

H11a,b: The perception of the information system's data accuracy will be positively associated with the worker's perception of significance in the: (a) legacy environment; (b) ERP environment.

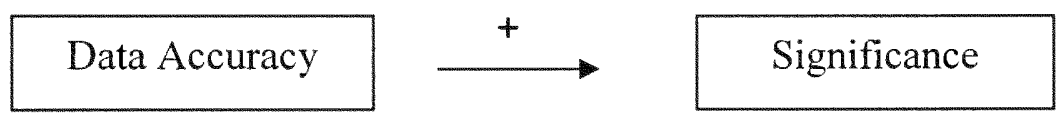

H12a,b: The perception of the information system's data accuracy will be positively associated with the worker's perception of working with others in the: (a) legacy environment; (b) ERP environment.

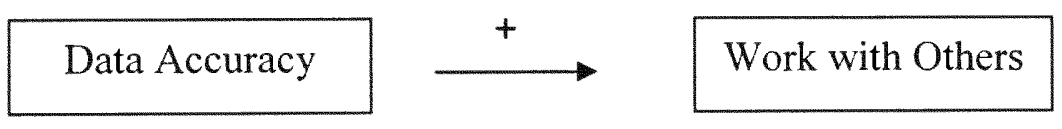

Compatibility

Data from several sources may be incompatible and not easily understood for the task at hand. This construct measures if the data is consistent across the many locations or systems. If the data is perceived to be compatible across several systems, then a positive relationship between data compatibility and the job design characteristics should be found. 
H13a,b: The perception of the information system's compatibility will be positively associated with the worker's perception of job variety in the: (a) legacy environment; (b) ERP environment.

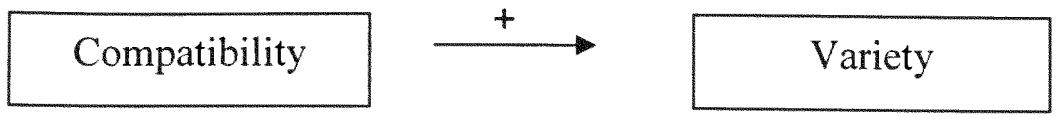

H14a,b: The perception of the information system's compatibility will be positively associated with the worker's perception of job identity in the: (a) legacy environment; (b) ERP environment.

\section{Compatibility}

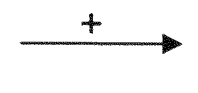

\section{Identity}

H15a,b: The perception of the information system's compatibility will be positively associated with the worker's perception of autonomy in the: (a) legacy environment; (b) ERP environment.

\section{Compatibility}

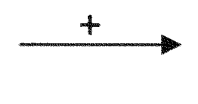

H16a,b: The perception of the information system's compatibility will be positively associated with the worker's perception of feedback in the: (a) legacy environment; (b) ERP environment.

Compatibility

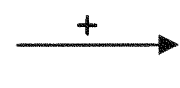

Feedback

H17a,b: The perception of the information system's compatibility will be positively associated with the worker's perception of significance in the: (a) legacy environment; (b) ERP environment.

Compatibility

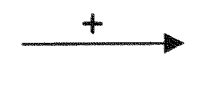

Significance

H18a,b: The perception of the information system's compatibility will be positively associated with the worker's perception of working with others in the: (a) legacy environment; (b) ERP environment.

Compatibility

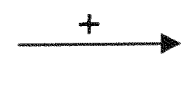

Work with Others 
The location of the data represents the user's ability to locate the data for use in the job. If the data is perceived to be easy to find to support the job, then a positive relationship between data location and the job design characteristics should be found.

H19a,b: The perception of the information system's data location will be positively associated with the worker's perception of job variety in the: (a) legacy environment; (b) ERP environment.

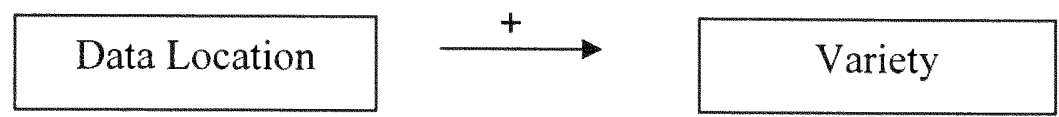

H20a,b: The perception of the information system's data location will be positively associated with the worker's perception of job identity in the: (a) legacy environment; (b) ERP environment.
Data Location

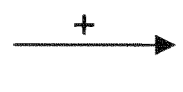
Identity

H21a,b: The perception of the information system's data location will be positively associated with the worker's perception of autonomy in the: (a) legacy environment; (b) ERP environment.

Data Location

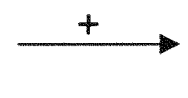

Autonomy

H22a,b: The perception of the information system's data location will be positively associated with the worker's perception of feedback in the: (a) legacy environment; (b) ERP environment.
Data Location

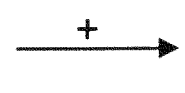
Feedback

H23a,b: The perception of the information system's data location will be positively associated with the worker's perception of significance in the: (a) legacy environment; (b) ERP environment.

Data Location

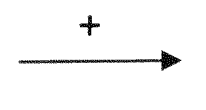

Significance 
$\mathrm{H} 24 \mathrm{a}$, b: The perception of the information system's data location will be positively associated with the worker's perception of working with others in the: (a) legacy environment; (b) ERP environment.

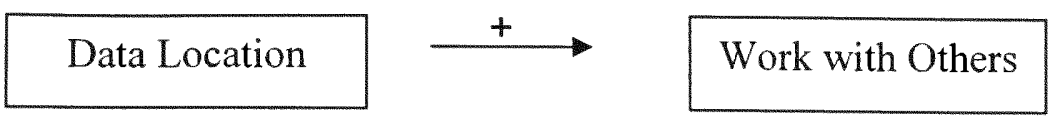

Data Accessibility

Data accessibility is the ability of the user to gain access to the data to support the job. If the data is perceived to be easy to gain access to in support of a task, then a positive relationship between data accessibility and the job design characteristics should be found.

H25a,b: The perception of the information system's data accessibility will be positively associated with the worker's perception of job variety in the: (a) legacy environment; (b) ERP environment.

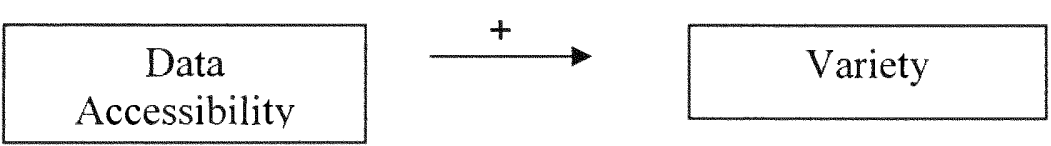

H26a,b: The perception of the information system's data accessibility will be positively associated with the worker's perception of job identity in the: (a) legacy environment; (b) ERP environment.

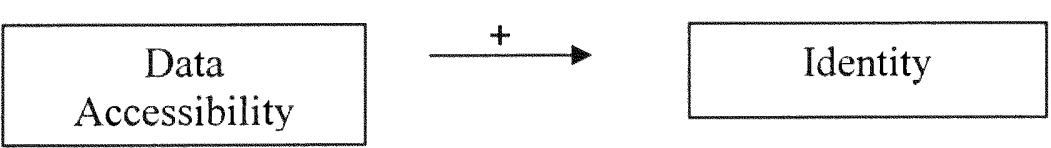

H27a,b: The perception of the information system's data accessibility will be positively associated with the worker's perception of autonomy in the: (a) legacy environment; (b) ERP environment.

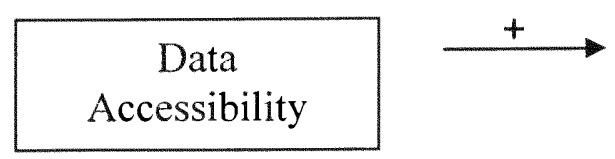

Autonomy 
H28a,b: The perception of the information system's data accessibility will be positively associated with the worker's perception of feedback in the: (a) legacy environment; (b) ERP environment.

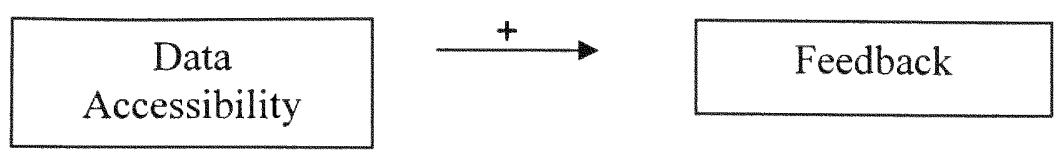

H29a,b: The perception of the information system's data accessibility will be positively associated with the worker's perception of significance in the: (a) legacy environment; (b) ERP environment.

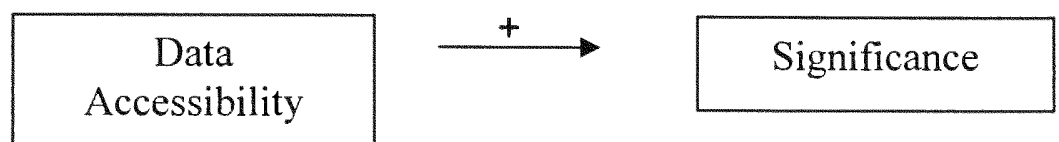

H30a,b: The perception of the information system's data accessibility will be positively associated with the worker's perception of working with others in the: (a) legacy environment; (b) ERP environment.

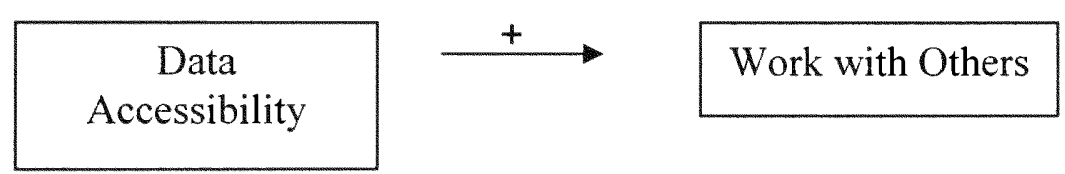

Data Meaning

Data meaning is the clarity with which data is represented. If the data is perceived to have the correct meaning to support a task, then a positive relationship between data meaning and the job design characteristics should be found.

H31a,b: The perception of the information system's data meanings will be positively associated with the worker's perception of job variety in the: (a) legacy environment; (b) ERP environment.

Data Meaning

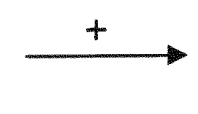

Variety 
H32a,b: The perception of the information system's data meanings will be positively associated with the worker's perception of job identity in the: (a) legacy environment; (b) ERP environment.

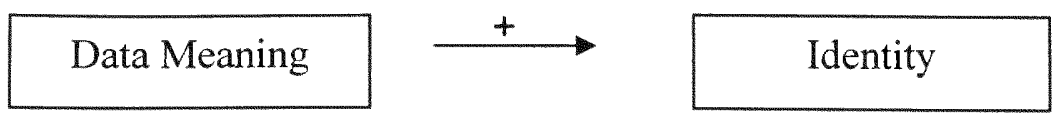

H33a,b: The perception of the information system's data meanings will be positively associated with the worker's perception of autonomy in the: (a) legacy environment; (b) ERP environment.

\section{Data Meaning}

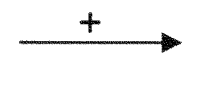

\section{Autonomy}

H34a,b: The perception of the information system's data meanings will be positively associated with the worker's perception of feedback in the: (a) legacy environment; (b) ERP environment.

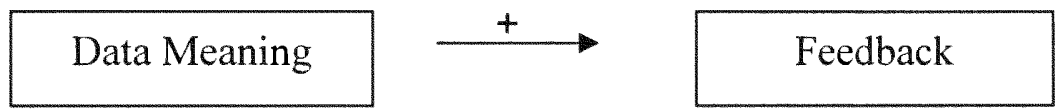

H35a,b: The perception of the information system's data meanings will be positively associated with the worker's perception of significance in the: (a) legacy environment; (b) ERP environment.
Data Meaning

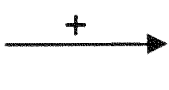
Significance

H36a,b: The perception of the information system's data meanings will be positively associated with the worker's perception of working with others in the: (a) legacy environment; (b) ERP environment.

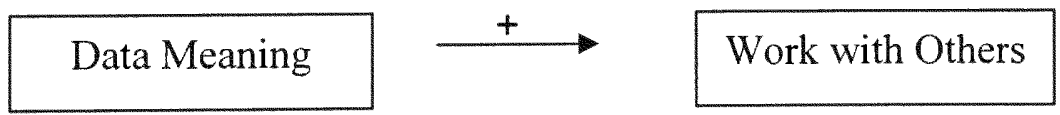

\section{Concurrency of Data}

Concurrency of data refers to how timely the information is to assist the task. If the data is perceived to be timely to support the job, then a positive relationship between data concurrency and the job design characteristics should be found. 
H37a,b: The perception of the information system's data concurrency will be positively associated with the worker's perception of job variety in the: (a) legacy environment; (b) ERP environment.

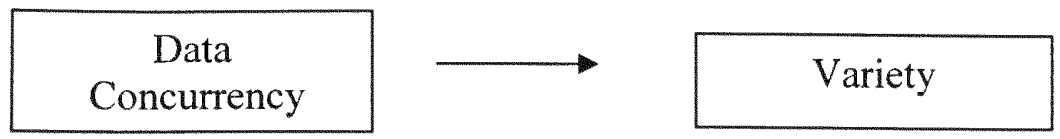

H38a,b: The perception of the information system's data concurrency will be positively associated with the worker's perception of job identity in the: (a) legacy environment; (b) ERP environment.

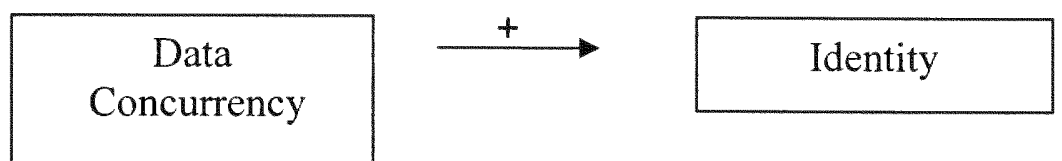

H39a,b: The perception of the information system's data concurrency will be positively associated with the worker's perception of autonomy in the: (a) legacy environment; (b) ERP environment.

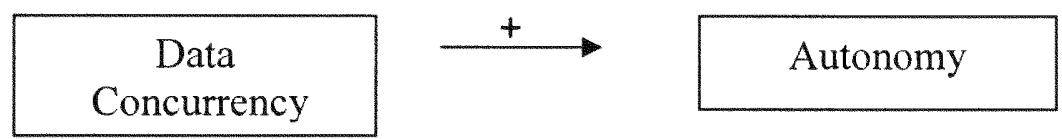

H40a,b: The perception of the information system's data concurrency will be positively associated with the worker's perception of feedback in the: (a) legacy environment; (b) ERP environment.

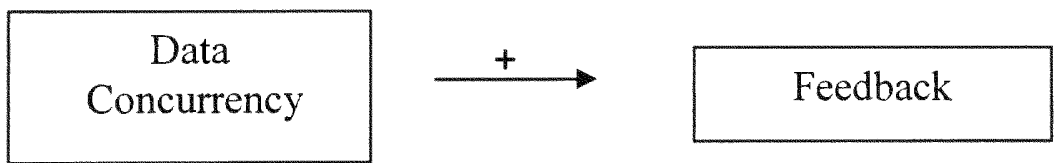

H41a,b: The perception of the information system's data concurrency will be positively associated with the worker's perception of significance in the: (a) legacy environment; (b) ERP environment.

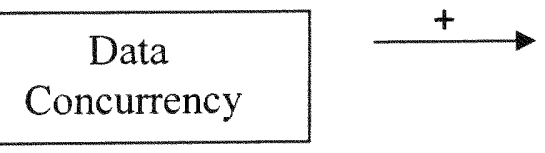

Significance 
H42a,b: The perception of the information system's data concurrency will be positively associated with the worker's perception of working with others in the: (a) legacy environment; (b) ERP environment.

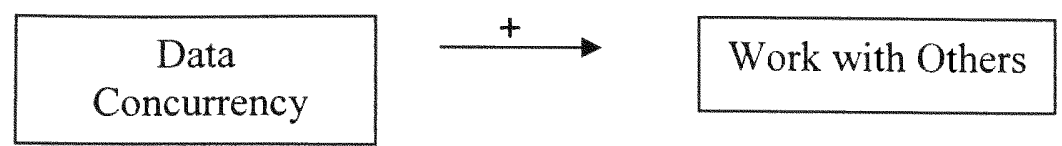

System Assistance

System assistance refers to problem resolution when using the system. The correct level of system assistance will support the job of the user. If the system assistance is perceived to have the correct level of support, then a positive relationship between system assistance and the job design characteristics should be found.

H43a,b: The perception of the information system's assistance will be positively associated with the worker's perception of job variety in the: (a) legacy environment; (b) ERP environment.

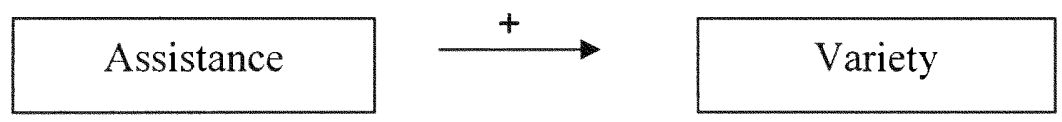

H44a,b: The perception of the information system's assistance will be positively associated with the worker's perception of job identity in the: (a) legacy environment; (b) ERP environment.

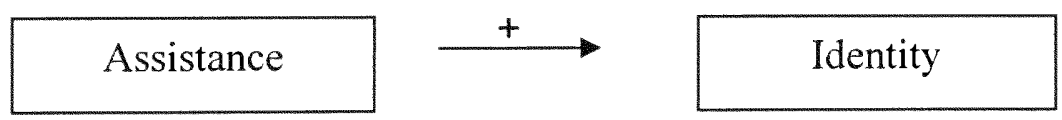

H45a,b: The perception of the information system's assistance will be positively associated with the worker's perception of autonomy in the: (a) legacy environment; (b) ERP environment.

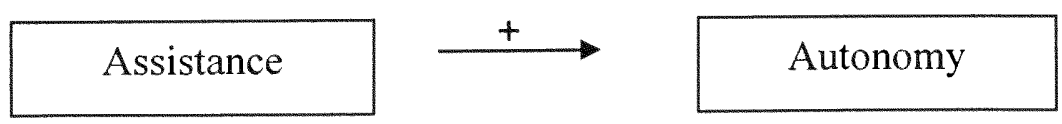


H46a,b: The perception of the information system's assistance will be positively associated with the worker's perception of feedback in the: (a) legacy environment; (b) ERP environment.

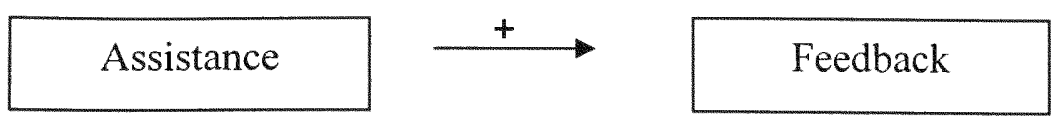

H47a,b: The perception of the information system's assistance will be positively associated with the worker's perception of significance in the: (a) legacy environment; (b) ERP environment.

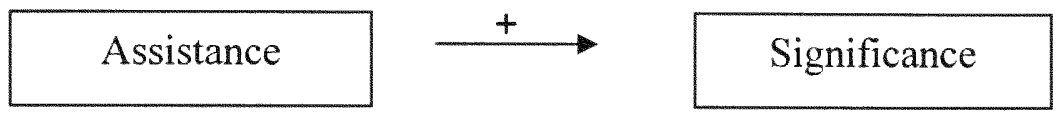

H48a,b: The perception of the information system's assistance will be positively associated with the worker's perception of working with others in the: (a) legacy environment; (b) ERP environment.

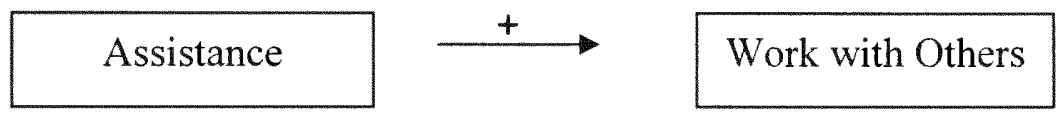

Ease of Use

The ease of use variable deals with how easy it is to use the information system to support the job. If the system is perceived to be easy to use, then a positive relationship between ease of use and the job design characteristics should be found.

H49a,b: The perception of the information system's data assistance will be positively associated with the worker's perception of job variety in the: (a) legacy environment; (b) ERP environment.

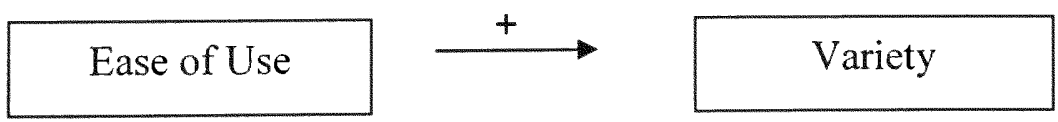


H50a,b: The perception of the information system's data assistance will be positively associated with the worker's perception of job identity in the: (a) legacy environment; (b) ERP environment.

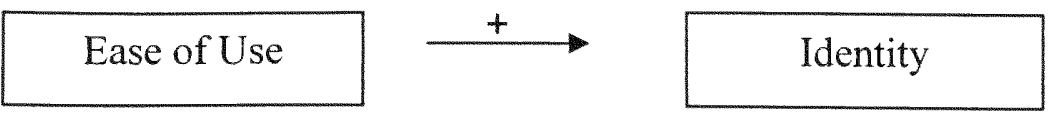

H51a,b: The perception of the information system's data assistance will be positively associated with the worker's perception of autonomy in the: (a) legacy environment; (b) ERP environment.

Ease of Use

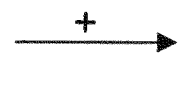

Autonomy

H52a,b: The perception of the information system's data assistance will be positively associated with the worker's perception of feedback in the: (a) legacy environment; (b) ERP environment.

$$
\text { Ease of Use }
$$

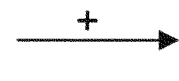

Feedback

H53a,b: The perception of the information system's data assistance will be positively associated with the worker's perception of significance in the: (a) legacy environment; (b) ERP environment.

Ease of Use

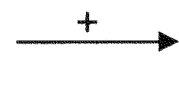

\section{Significance}

H54a,b: The perception of the information system's data assistance will be positively associated with the worker's perception of working with others in the: (a) legacy environment; (b) ERP environment.

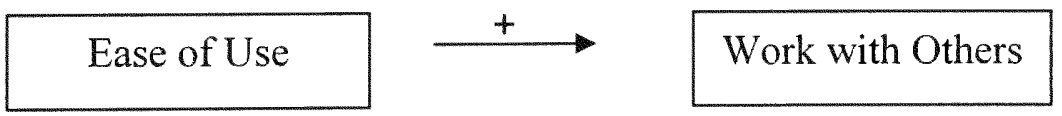

Reliability

The dependability of the information system in supporting the job is the reliability variable. If the system is perceived to be reliable, then a positive relationship between system reliability and the job design characteristics should be found. 
H55a,b: The perception of the information system's reliability will be positively associated with the worker's perception of job variety in the: (a) legacy environment; (b) ERP environment.

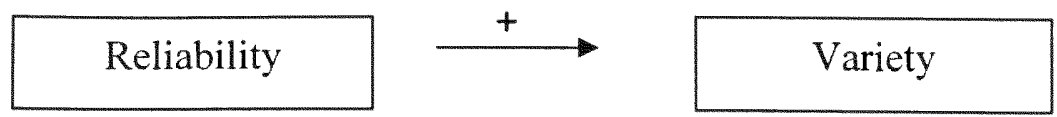

H56a,b: The perception of the information system's reliability will be positively associated with the worker's perception of job identity in the: (a) legacy environment; (b) ERP environment.

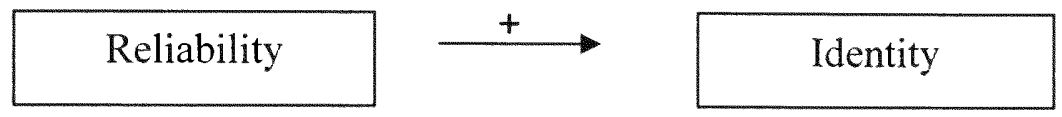

H57a,b: The perception of the information system's reliability will be positively associated with the worker's perception of autonomy in the: (a) legacy environment; (b) ERP environment.

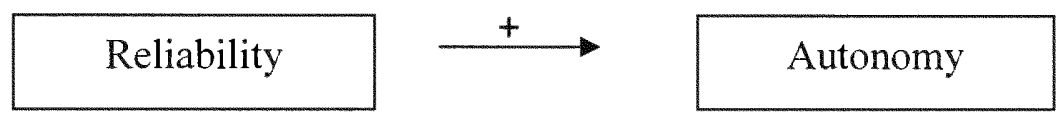

H58a,b: The perception of the information system's reliability will be positively associated with the worker's perception of feedback in the: (a) legacy environment; (b) ERP environment.

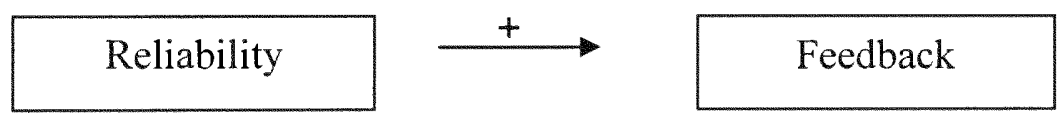

H59a,b: The perception of the information system's reliability will be positively associated with the worker's perception of significance in the: (a) legacy environment; (b) ERP environment.

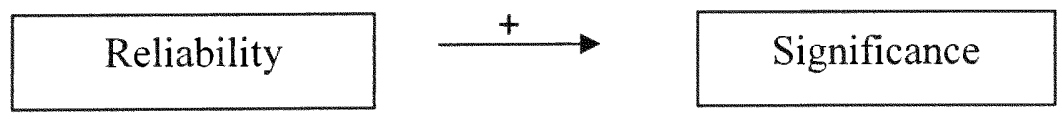

H60a,b: The perception of the information system's reliability will be positively associated with the worker's perception of working with others in the: (a) legacy environment; (b) ERP environment.

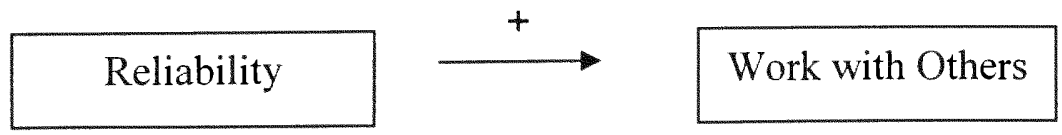


Presentation

Presentation refers to the display of data on the screen and in reports and how easy it is to interpret and use. If the system presentation is positively perceived to be a support of the job task, then a positive relationship between system presentation and the job design characteristics should be found.

H61a,b: The perception of the information system's presentation will be positively associated with the worker's perception of job variety in the: (a) legacy environment; (b) ERP environment.

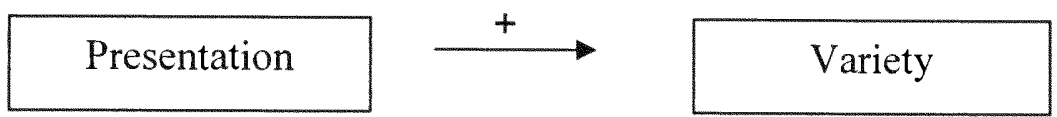

H62a,b: The perception of the information system's presentation will be positively associated with the worker's perception of job identity in the: (a) legacy environment; (b) ERP environment.

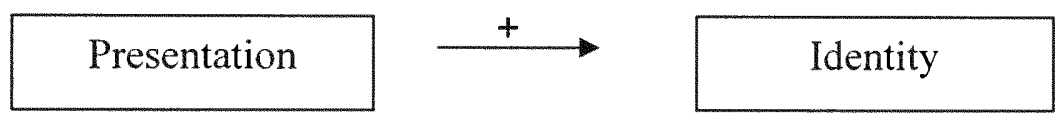

H63a,b: The perception of the information system's presentation will be positively associated with the worker's perception of autonomy in the: (a) legacy environment; (b) ERP environment.

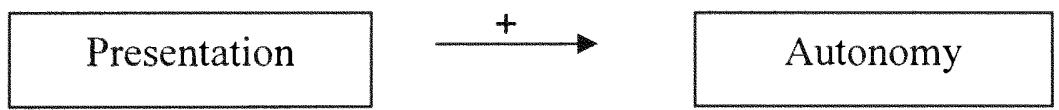

H64a,b: The perception of the information system's presentation will be positively associated with the worker's perception of feedback in the: (a) legacy environment; (b) ERP environment.

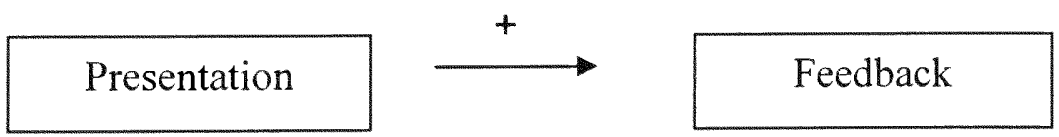

H65a,b: The perception of the information system's presentation will be positively associated with the worker's perception of significance in the: (a) legacy environment; (b) ERP environment.

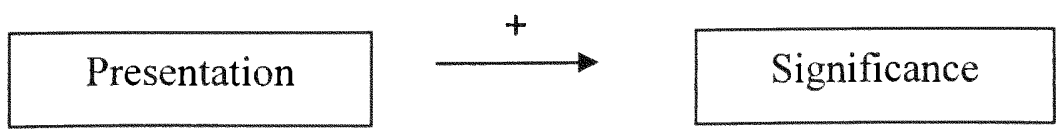


H66a,b: The perception of the information system's presentation will be positively associated with the worker's perception of working with others in the: (a) legacy environment; (b) ERP environment.
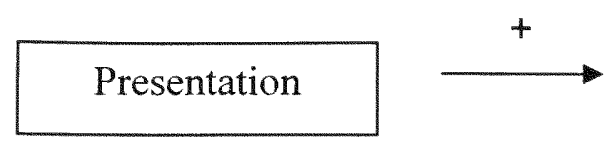

\section{Work with others}

Confusion

System confusion is the result of a system's poor organization or a lack of understanding of the system by the user doing the task. If the system is perceived to be confusing and unable to support the job task, then a negative relationship between system confusion and the job design characteristics should be found.

H67a,b: If the perception of the information system is confusing to the user, it will be negatively associated with the worker's perception of job variety in the: (a) legacy environment; (b) ERP environment.

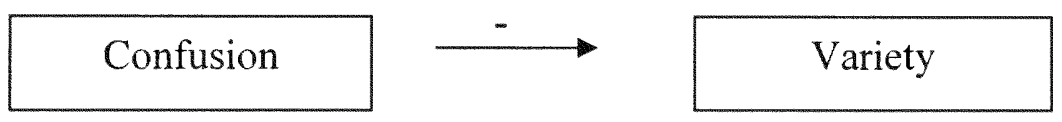

H68a,b: If the perception of the information system is confusing to the user, it will be negatively associated with the worker's perception of job identity in the: (a) legacy environment; (b) ERP environment.

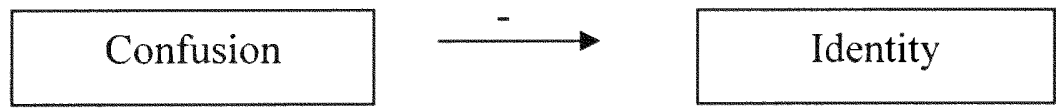

H69a,b: If the perception of the information system is confusing to the user, it will be negatively associated with the worker's perception of job autonomy in the: (a) legacy environment; (b) ERP environment.

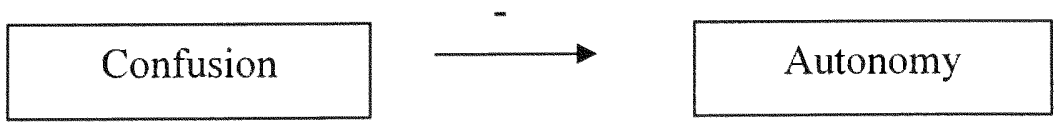

H70a,b: If the perception of the information system is confusing to the user, it will be negatively associated with the worker's perception of the job feedback in the: (a) legacy environment; (b) ERP environment.

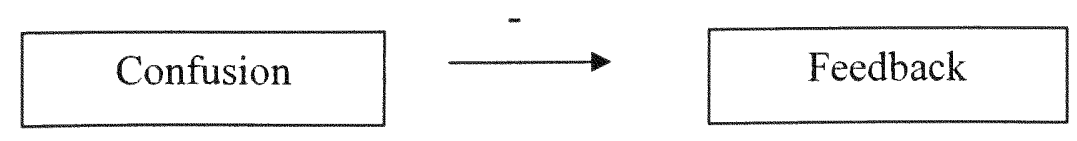


H71a,b: If the perception of the information system is confusing to the user, it will be negatively associated with the worker's perception of job significance in the: (a) legacy environment; (b) ERP environment.

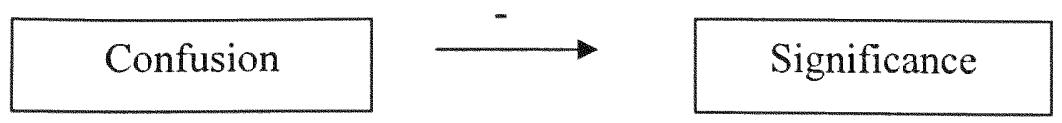

H72a,b: If the perception of the information system is confusing to the user, it will be negatively associated with the worker's perception of working with others in the: (a) legacy environment; (b) ERP environment.

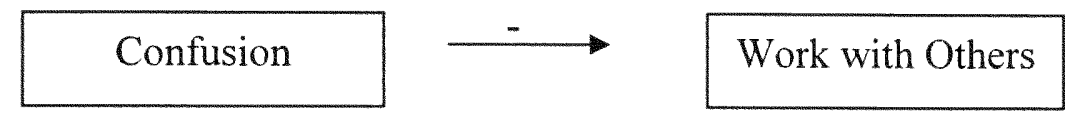

The first set of questions detailed design can be seen in Figure 3.

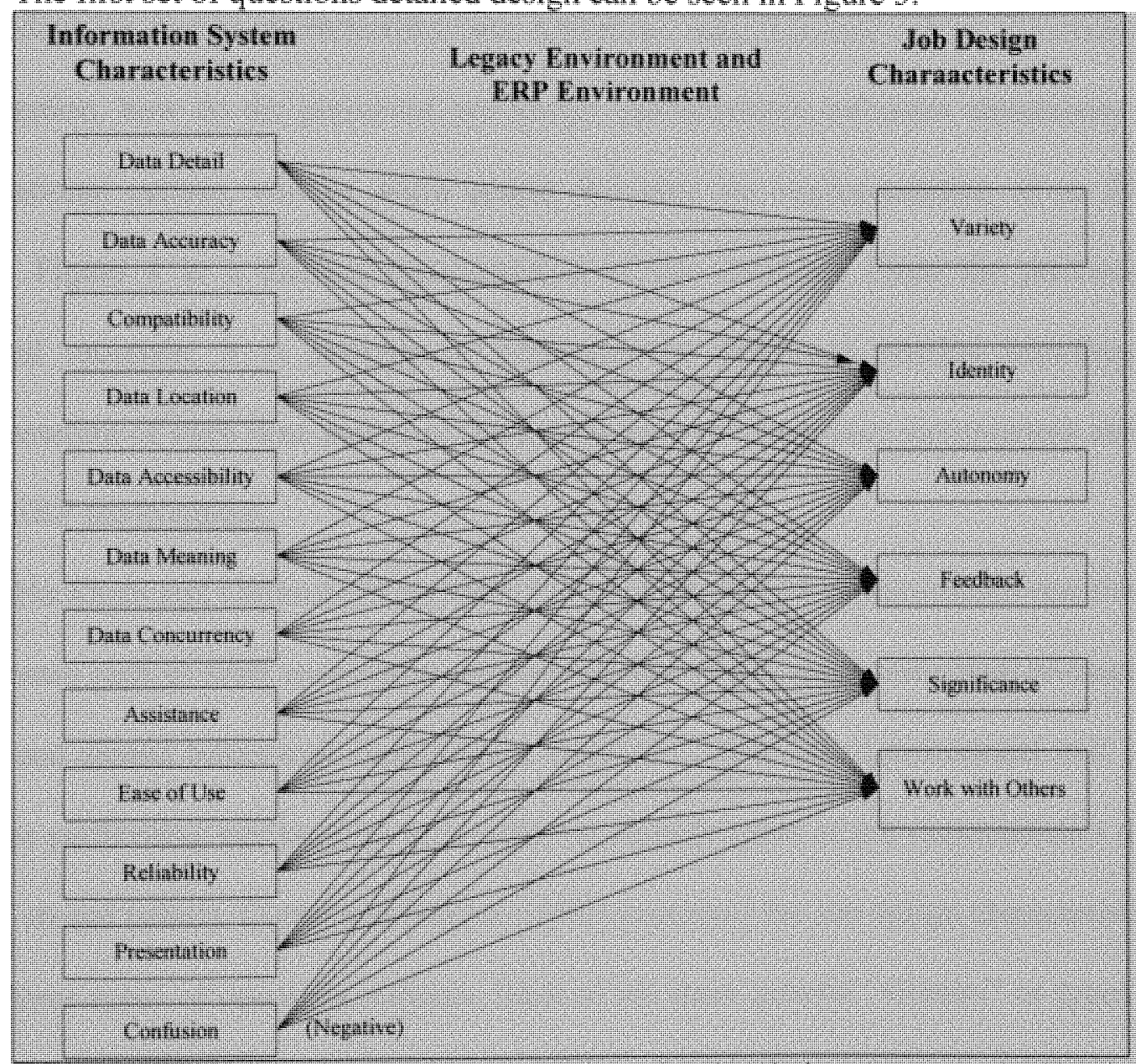

Figure 3 - Detailed model of system and job characteristics survey variables 
The third question compares job characteristics and their linkages to information system characteristics between the legacy time period and the ERP time period. The hypothesis question to be asked:

3H1: Job design perceptions and job perception linkages to information system characteristics found in the legacy time period will be different in the ERP time period.

\section{Research Question Set \#2}

The two questions in the second set on page 50 examine the association between independent job design characteristics and dependent organizational culture dimensions. The question, how do the perceived job design characteristics influence dimensions of organizational culture, will be asked before and after the implementation of an ERP system. Figure 4 is a detailed representation of the variables. From the previously discussed rationale, it is possible to formulate the following detailed hypotheses. Representation of each time period in designated by $a$ for the legacy environment and $b$ for the ERP environment.

Hackman and Oldman (1974) identified a set of core job characteristics that relate to outcomes such as work motivation and performance. These shape individual normative beliefs and shared behavioral expectations, especially when the organizational unit has members performing similar jobs. In an effort to identify types of job characteristics that support shared beliefs, Cooke and Szumal (1993) examined the job design characteristics within three types of operating cultures. Each of the three general "clusters" or types of organizational culture is labeled constructive, passive/defensive, or aggressive/defensive. 


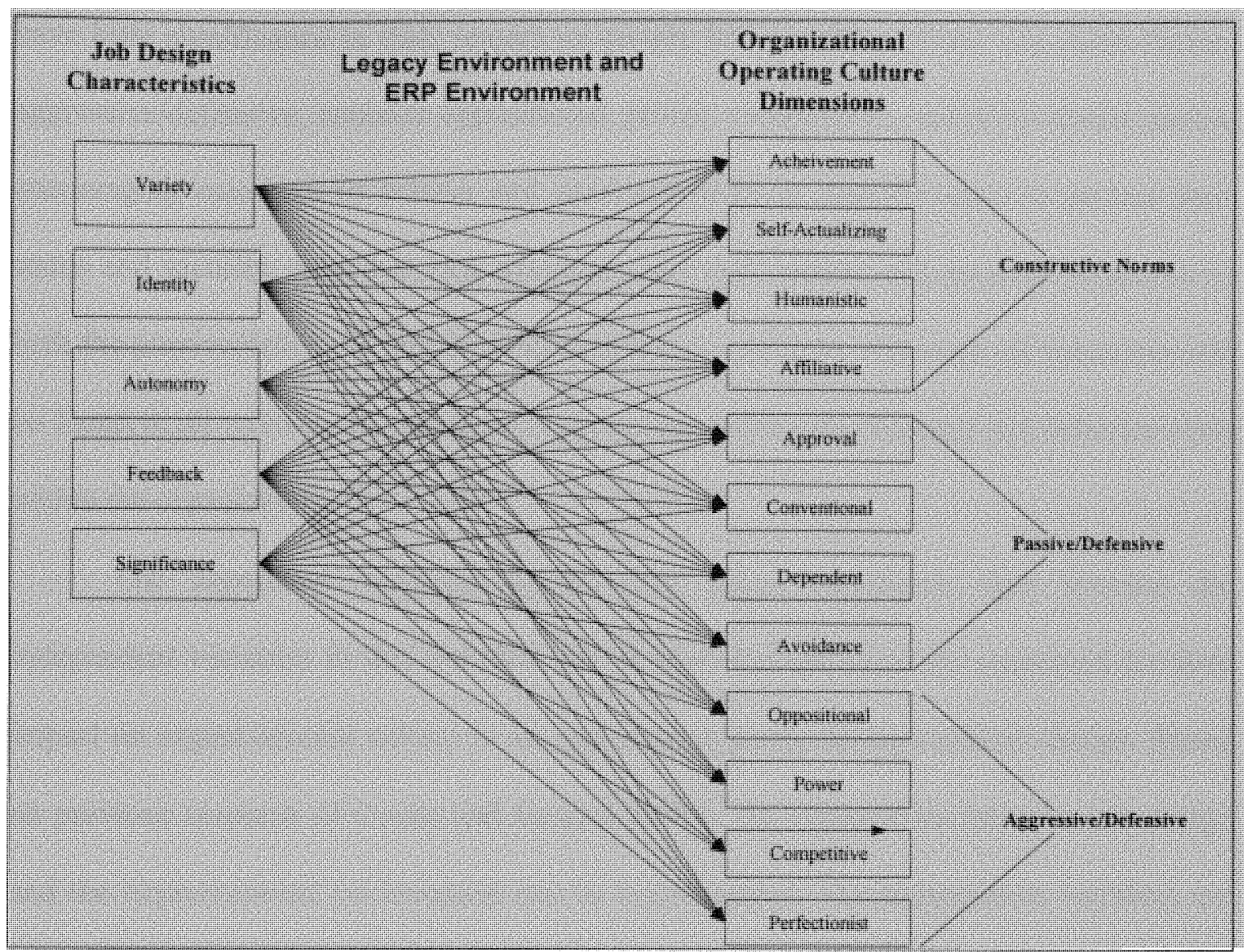

Figure 4 - Detailed model of job characteristics and culture dimensions survey variables

These clusters are the result of twelve sets of norms that distinguished between two underlying dimensions: the concern for people and the concern for task. In their analysis, the researchers found jobs that provide high levels of autonomy, skill variety, task identity, task significance, and feedback are positively associated with constructive norms. Constructive norms are characterized as achievement, self-actualizing, humanistic-encouraging, and affiliate behaviors. Workers approach tasks and interact with other workers in ways that will help them meet their higher-order satisfaction. When workers are required or encouraged to interact in ways that will not threaten their own personal security, then the culture is labeled passive/defensive. This culture is characterized by approval, conventional, dependent, and avoidance behaviors. Jobs that 
have little significance in their impact on people fall into this culture. The third cluster, aggressive/defensive, contains oppositional, power, competitive, and perfectionist norms, encouraging members to behave and approach tasks in forceful ways to protect their status and security. Jobs that lack autonomy and skill variety will promote behaviors in both the passive/defensive and aggressive/defensive cultures. Although all behaviors will be found in all cultures, the strength of the norms defines the thinking and behavior style within the organization.

The dependent variables of the first major hypothesis now become the independent variables in this question. Job design characteristics are examined for their impact on organizational culture in an organization going through radical change. Based on previous research of Cooke and Szumal (2000), the connections between job design and the organizational environment can be hypothesized. The independent variables are job variety, job identity, task significance, job autonomy, and job feedback ${ }^{9}$. (For a detailed discussion of the variables, refer to major hypothesis question I.)

The dependent variables will be the twelve dimensions of behavior norms of the organizational culture inventory (OCI) instrument. The twelve dimensions are grouped into three clusters. The first group, labeled constructive, included the behavioral norms of achievement, self-actualization, humanistic-encouraging, and affiliate. Achievement refers to how members are expected to set challenging but realistic goals, establish plans to reach goals, and pursue them with enthusiasm. When members are expected to enjoy their work, develop themselves, and take on new and interesting tasks, they are exhibiting

\footnotetext{
${ }^{9}$ The "work with others" JDS variable is not used in the second question. It was not studied in the research of Cooke and Szumal (2000).
} 
self-actualizing behavior. Humanistic-encouraging norms are the expectation that members will be supportive, constructive, and open to influence in their dealings with one another. Sensitivity, friendliness, and cooperation are behaviors of the affiliate norm.

The second and third compose the defensive norms groups. Passive/defensive norms are approval, conventional, dependent, and avoidance. In this group, members are encouraged to act in ways that will not threaten their own personal security. Approval norms are the members expecting to agree with, gain the approval of, and be liked by others in the organization. When members are expected to conform, follow the rules, and make a good impression, then the conventional norms are being observed. Dependent behavior dictates that the members do what they are told and that all decisions must be cleared with superiors. Avoidance norms are the members expecting to shift responsibilities to others and avoid any possibility of being blamed for problems. Aggressive/defensive norms are oppositional, power, competitive, and perfectionistic. Oppositional norms are the members expecting to be critical, opposed to the ideas of others, and make safe, although ineffectual, decisions. The members are expected to take charge, control subordinates, and yield to the demands of superiors in power. Competitive behavior encourages members to operate in "win-lose" frameworks, outperform others, and work against their peers. Perfectionist norms expect members to appear competent, keep track of everything, and work long hours to attain narrowlydefined objectives.

The following hypotheses are created to support the study's second set of questions. 
Job Variety

Job variety is the degree to which the job requires many different skills and talents of the worker (Hackman and Oldham, 1974). Based on the previous research of Cooke and Szumal (2000), jobs that are simple and routine establish norms for compliant and "detached" behaviors. This type of behavior suppresses expectations for achievement, growth, and collaboration. This leads to job variety being positively associated with achievement, self-actualizing, humanistic-encouraging, and affiliate behaviors. If job variety is lacking, a negative association will be found with passive/defensive and aggressive/defensive culture dimensions of approval, conventional, dependent, avoidance, oppositional, power, competitive, and perfectionist behaviors.

H73a,b: Job variety is positively associated with constructive norms (achievement, selfactualizing, humanistic-encouraging, and affiliate behaviors) in the: (a) legacy environment; (b) ERP environment.

Job Variety

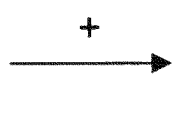

Constructive Norms

Achievement

Self-actualizing

Humanistic-encouraging

Affiliate 
H74a,b: Job variety is negatively associated with passive/defensive norms (approval, conventional, dependent, and avoidance behaviors) in the: (a) legacy environment; (b) ERP environment.

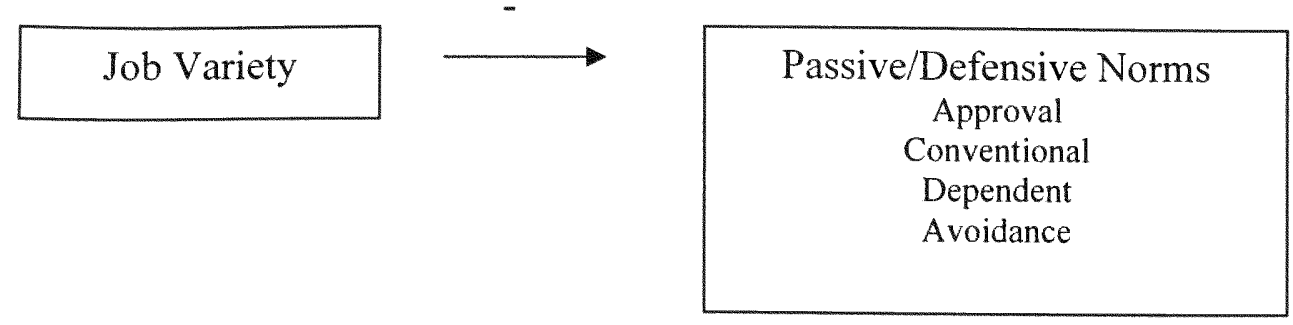

H75a,b: Job variety is negatively associated with aggressive/defensive norms (oppositional, power, competitive, and perfectionist behaviors) the: (a) legacy environment; (b) ERP environment.

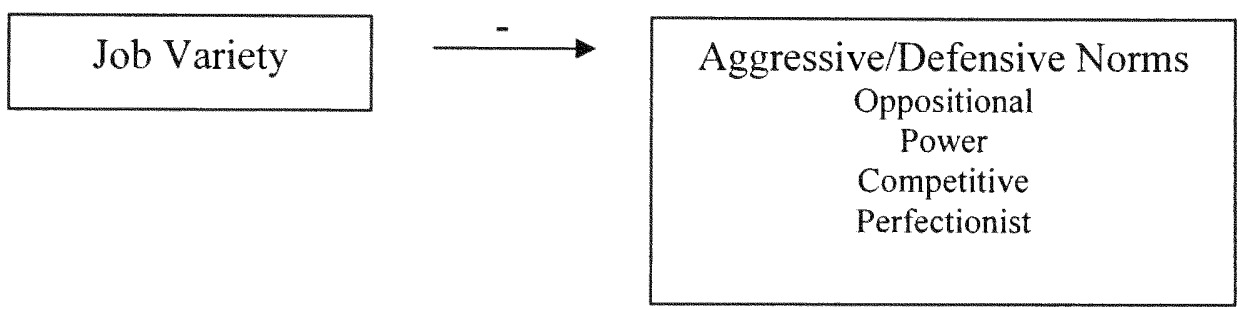

Job Identity

Job identity is the extent to which the job encompasses an entire piece of work or only makes a small contribution to a larger work effort. In a constructive environment, job identity is positively associated with achievement, self-actualizing, humanisticencouraging, and affiliate behaviors. If job identity is lacking, no association will be found in the passive/defensive and aggressive/defensive dimensions of approval, conventional, dependent, avoidance, oppositional, power, competitive, and perfectionist behaviors. These relationships are based on the previous research of Cooke and Szumal $(2000)$. 
H76a,b: Job identity is positively associated with constructive norms (achievement, self actualizing, humanistic-encouraging, and affiliate behaviors) in the: (a) legacy environment; (b) ERP environment.

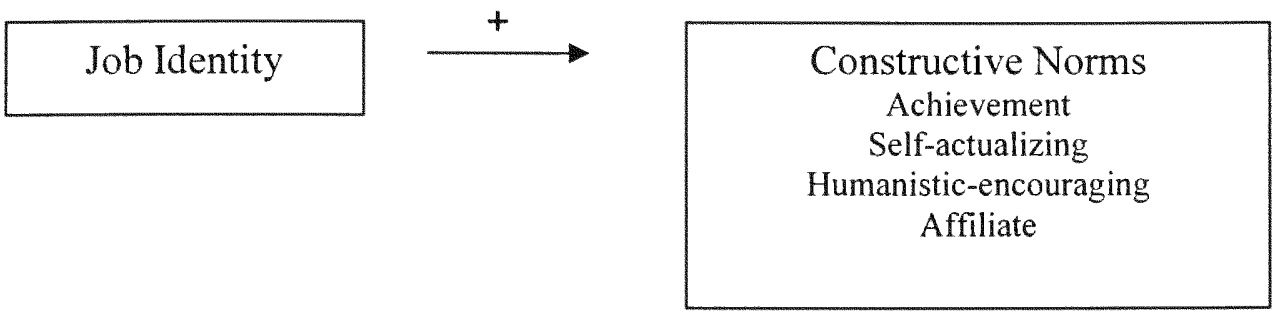

H77a,b: Job identity is not found in cultures characterized by passive/defensive norms (approval, conventional, dependent, and avoidance behaviors) in the: (a) legacy environment; (b) ERP environment.
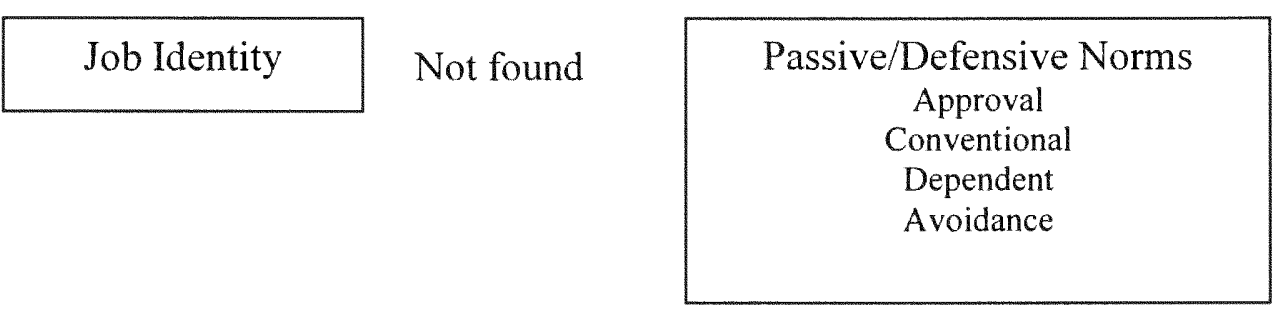

H78a,b: Job identity is not found in cultures characterized by aggressive/defensive norms (oppositional, power, competitive, and perfectionist behaviors) in the: (a) legacy environment; (b) ERP environment.

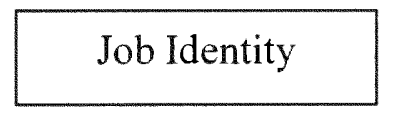

Not found

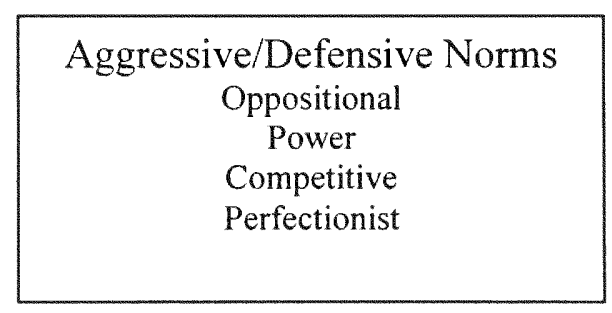

Task Significance

Task or job significance is the importance of the job in affecting the broader scheme of things. Based on the previous research of Cooke and Szumal (2000), jobs that have little significance in terms of their impact on people are associated with passive/defensive behaviors and in the opposite, jobs with a high degree of impact are associated with constructive behaviors. Task significance is positively associated with 
achievement, self-actualizing, humanistic-encouraging, and affiliate behaviors. In both defensive environments, a negative association will be between task or job significance and the dimensions of approval, conventional, dependent, avoidance, oppositional, power, competitive, and perfectionist behaviors is found.

H79a,b: Task significance is positively associated with constructive norms (achievement, self-actualizing, humanistic-encouraging, and affiliate behaviors) in the: (a) legacy environment; (b) ERP environment.
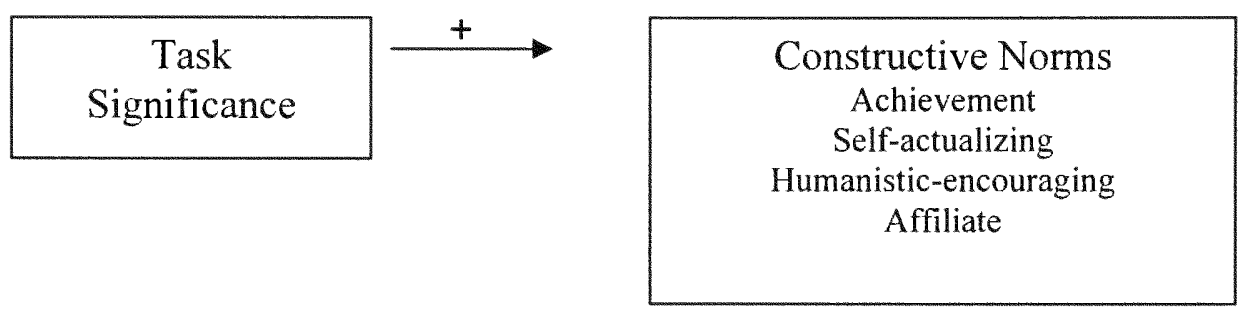

H80a,b: Task significance is negatively associated with passive/defensive norms (approval, conventional, dependent, and avoidance behaviors) in the: (a) legacy environment; (b) ERP environment.
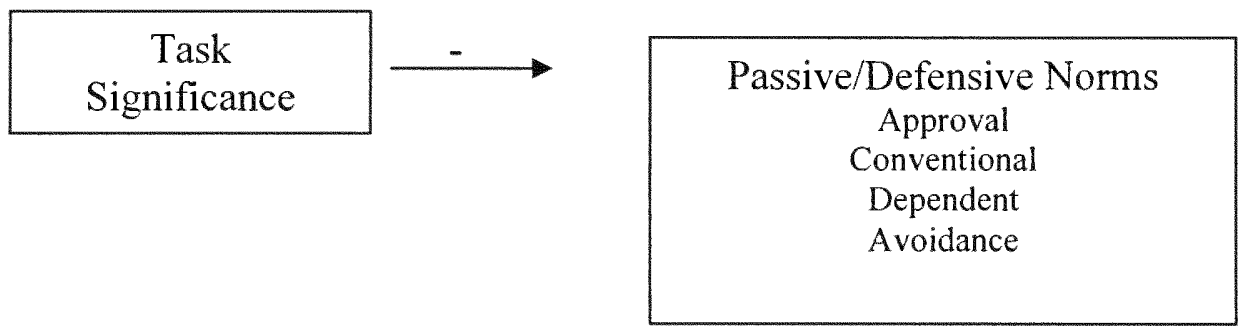

H81a,b: Task significance is negatively associated with aggressive/defensive norms (oppositional, power, competitive, and perfectionist behaviors) in the: (a) legacy environment; (b) ERP environment.
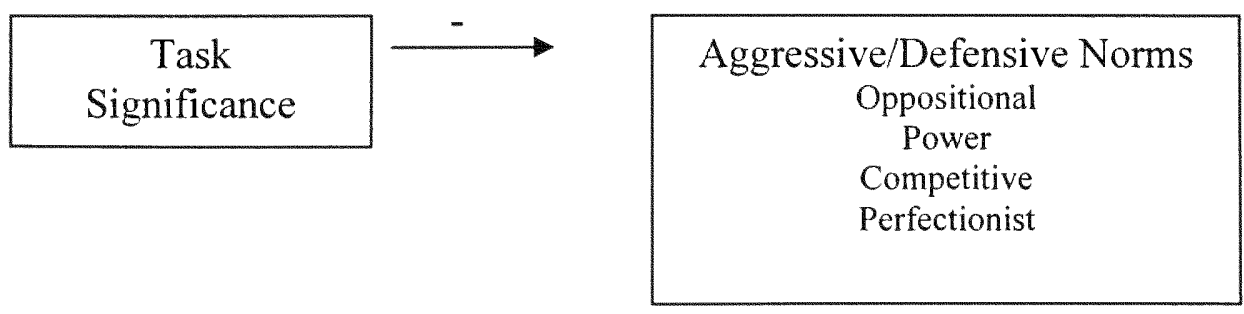
Job Autonomy

Job autonomy is if the job provides substantial freedom or discretion for the employee to schedule and accomplish the work. Jobs that provide high levels of autonomy are positively associated with achievement, self-actualizing, humanisticencouraging, and affiliate behaviors. In both defensive environments, a negative association will be found with job autonomy and the dimensions of approval, conventional, dependent, avoidance, oppositional, power, competitive, and perfectionist behaviors. These relationships are based on the previous research of Cooke and Szumal $(2000)$.

H82a,b: Job autonomy is positively associated with constructive norms (achievement, self-actualizing, humanistic-encouraging, and affiliate behaviors) in the: (a) legacy environment; (b) ERP environment.
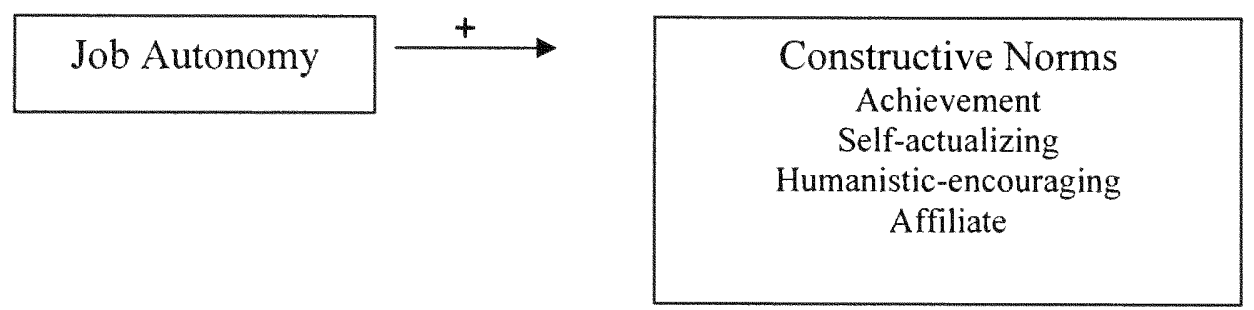

H83a,b: Job autonomy is negatively associated with passive/defensive norms (approval, conventional, dependent, and avoidance behaviors) in the: (a) legacy environment; (b) ERP environment.

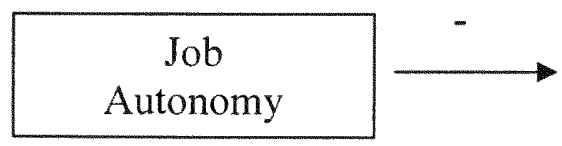

\begin{tabular}{|c|}
\hline Passive/Defensive Norms \\
Approval \\
Conventional \\
Dependent \\
Avoidance \\
\hline
\end{tabular}


H84a,b: Job autonomy is negatively associated with aggressive/defensive norms (oppositional, power, competitive, and perfectionist behaviors) in the: (a) legacy environment; (b) ERP environment.

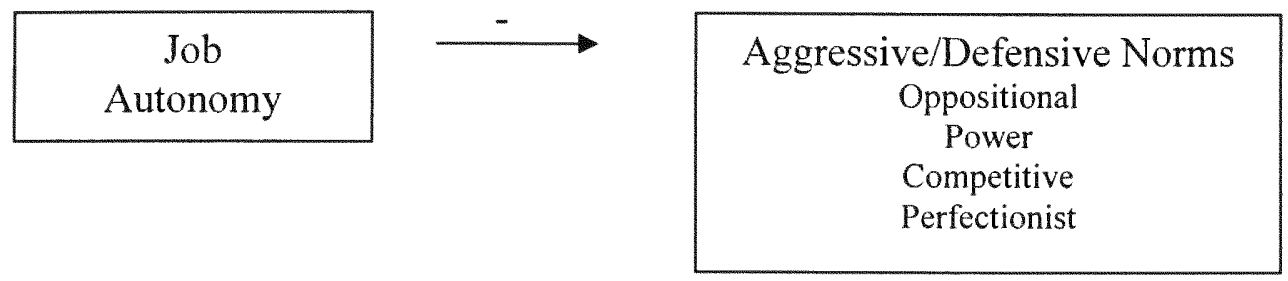

Job Feedback

Job feedback is the extent to which the job itself provides information about the worker's performance. Based on the previous research of Cooke and Szumal (2000), in a constructive environment, job feedback is positively associated with achievement, selfactualizing, humanistic-encouraging, and affiliate behaviors. In both defensive environments, a negative association will be found with job feedback and the dimensions of approval, conventional, dependent, avoidance, oppositional, power, competitive, and perfectionist behaviors.

H85a,b: Job feedback is positively associated with constructive norms (achievement, self-actualizing, humanistic-encouraging, and affiliate behaviors) in the: (a) legacy environment; (b) ERP environment.

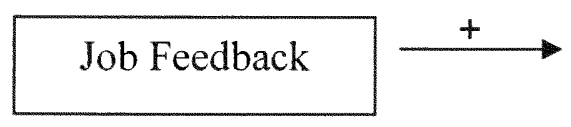

\begin{tabular}{|c|}
\hline Constructive Norms \\
Achievement \\
Self-actualizing \\
Humanistic-encouraging \\
Affiliate \\
\hline
\end{tabular}


H86a,b: Job feedback is negatively associated with passive/defensive norms (approval, conventional, dependent, and avoidance behaviors) in the: (a) legacy environment; (b) ERP environment.

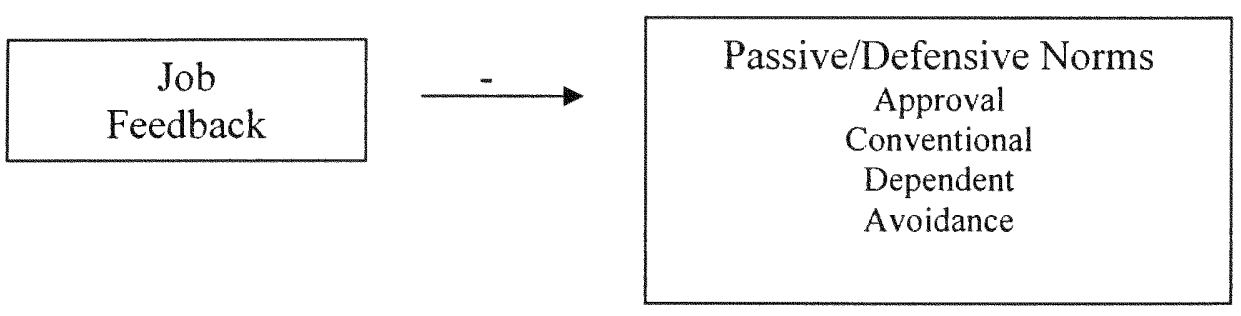

H87a,b: Job feedback is negatively associated with aggressive/defensive norms (oppositional, power, competitive, and perfectionist behaviors) in the: (a) legacy environment; (b) ERP environment.
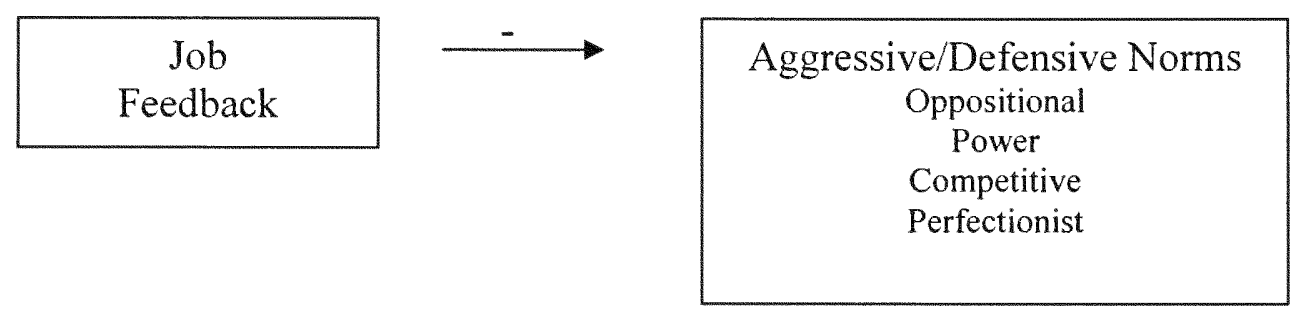

The third question compares job design characteristics and their linkages to organizational culture dimensions between the legacy time period and the ERP time period. The hypothesis question to be asked:

3H2: Job design perceptions and job perception linkages to organizational culture dimensions found in the legacy time period will be different in the ERP time period.

In this chapter, the design of the research model was described by three components: information system characteristics, job design characteristics, and organizational operating culture dimensions. From the relationship found in chapter three, twelve system characteristics and five job design specific independent variables were derived. Hypotheses were developed in this chapter regarding the association each of these variables might have on job design and organizational culture dimensions. 
Appendix A provides a summary of the detailed hypotheses. The methods used in this study are discussed in the next section.

\section{Method}

The design of the study follows a modified quasi-experimental approach in a field setting. As described by Shadish and colleagues (2002), the purpose of experiments is to test descriptive premises about causes that can be manipulated or treated. Without the treatment, the cause would not be present. Quasi-experiments lack the random assignment of the treatment. For this study, the target population of the study was all of the employees in the selected departments; therefore, representative sampling was not used. The timing of when to measure the treatment is a critical element of this type of experiment. It was important to gain a perceptive about how each participant viewed the information systems environment characteristics, job design characteristics, and organization operating culture dimensions during the two time periods. The treatment was the replacement of an organizational structure element, the legacy information system, with the new ERP information system. To examine the effect of the treatment, the study measured the operating environment during the legacy information system usage and during the new ERP information system usage. The experiment method modification was the absence of a control group. Due to the nature of the work setting, all departments of the organization were exposed to the treatment and therefore unable to represent an unexposed control group.

Studying in chaotic, changing organizations introduces threats of validity to the research by introducing many environment variables. One way to reduce these threats is 
to include only one organization in the study. This localizes the results but limits the future application of generalizing the causal and effect outcomes. To overcome these shortcomings, the participation of the entire affected population in one case study increases the measured credibility of the variables and reduces bias impacts of the nonmeasured latent variables. In this study, one organization was used as the case study and the selected departments had nearly complete participation. Although the applicability of the study may be limited to similar case settings, the effects found are valid for the entire population.

Research Study Background

A public state University implementing an academic version of an ERP system was selected for the study. The thirty year-old University is located in the southern part of the United States and currently has 1,100 faculty and staff, and over 34,000 students enrolled in undergraduate and graduate studies.

Due to external forces such as decreasing state funding and rapid enrollment growth, the University had been an organization in crisis. Each college had tried to take control of their own future and implemented different strategic plans for funding, marketing, and management of college resources. The support organizations (such as the ones in the study) had been coping with the changes but did not have a unified leadership or strategy to proactively deal with the impact of the changes until it was decided to implement the new system. The replacement of the old system was a forced action on the part of the state government. Each of the state system universities were informed that the state run legacy computer system was going to be retired and their institution was 
responsible for creating their own computing solution. University management decided that a new system would be purchased and installed. The new system was regarded as a way to unify the silos of support departments and create a better workplace model. This workplace change was not a planned effort by the management, but looked at as a direct result of the system implementation. Beyond the system change, no other organizational structural changes were planned.

Due to the unique processing requirements of the university settings, there are only a limited number of computing solutions available in the marketplace. The system chosen to replace the state system was the ERP product from the American company

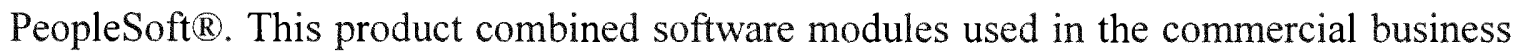
setting such as accounting and human resources with an academic module designed to support student services. The ERP system implementation was executed in phases based on the system module and supporting functionality over a three-year period. The first phase consisted of a limited set of student services including some admissions and financial aid functionality. The remaining legacy system's student service functionality and the financial administration module were replaced in the second phase.

The research timeline paralleled the system implementation timing. An initial research presentation describing the objectives of the study was made in meetings with university executives before the deployment of the ERP system. The executives agreed to allow two of the largest departments to participate based on the department members' interaction with the information system. Each of the departments self-administered and controlled their work activities without outside authority. The two University support departments chosen to participate in the study were selected based on their expected 
exposure to the new system. Each department was expected to experience a high degree of work task and process change using the new system.

The first department (admissions, registrars, and financial aid office known as student administration) had different senior managers but reported to the same executive vice president. The physical setting for student administration was in the same general area. The second department, the controller's office, reported to a different executive vice president (responsible for financial processes) and had a turnover of senior managers after the first survey data collection. Personnel for the controller's office were located in two office spaces. The first space was shared with the financial aid office and the second is located in a separate building across campus.

The department leaders provided their cooperation, access to their department members, and a listing of key informants they thought would be good participants in the informal discussions and semi-structured interviews.

\section{Data Collection Methods}

Initial versions of the questionnaire were pre-tested with approximately 25 people for readability and timing. Minor revisions were made to the survey format. The survey packets (containing one copy of each survey) were delivered via the employee's desktop to ensure that the respondents received the packet and tracked to ensure that all members responded. Participants created their own personal identification number (PIN) to identify themselves in the latter survey. The PIN owner was not known but the researcher was still able to pair the two different measurement period responses for analysis. 
Completing the survey took approximately 45 minutes. The study was administered to all employees (with the exception of student work/study employees) below middle management, which included all of the processing, clerical, administrative, support, and direct supervisor personnel. The participants answered the survey twice: the first time, while using the legacy system to support the work environment and the second time after the new ERP system replaced the legacy in support of the work environment. Semi-structured interviews also were used to gather information but were not included as part of the data analysis.

Demographic Information

The survey was administered on paper due to specific copyright permissions. Demographic information was gathered on each participant by two methods. University department and PIN were asked for on the first page of the survey. Age, sex, ethnic background, education, organizational level, salary, years with the organization, organization type, and profession/occupation responses was collected on the OCI instrument. Table 3 presents some demographic information for the participants in the survey.

Data Analysis Preparation

Before the data could be used in analysis, it was screened for accuracy and completeness. Also, basic data assumptions were met for appropriate use in further analysis. Individual statistical procedure assumptions were addressed separately by procedure. Each of these issues is discussed in the next sections. 
In order to ensure accuracy of data entry, the data was checked by two people. The first individual keyed the survey responses and the second validated the input. The roles were reversed and the input was verified a second time. Several random samples were pulled from the complete set of questionnaires and their corresponding records in the spreadsheet files were double checked. Using the procedure, only $2 \%$ of the first measurement and $1.5 \%$ of the second measurement were identified as having mismatches. They were corrected using the original responses.

One hundred thirty three responses were collected in the first measurement and 125 responses in the second measurement. One hundred thirty cases were retained in the first measurement after three cases were dropped due to lack of valid data, and 109 cases were retained in the second measurement for similar reasons. Ninety-nine individuals participated in both measurement period and were able to be tracked by their PIN number. Missing data for both measurements ranged from $0-2 \%$ per item. All variables were retained in the analysis. Survey participation averaged above $85 \%$ of the department populations ${ }^{10}$ for each measurement period. The high participation rate is attributed to department coordinators' efforts and the encouragement of this researcher. Table 4 reports participation by department.

\footnotetext{
${ }^{10}$ The controller's office numbers reflect a staff reduction due to a voluntary early retirement program. The vacancies were not filled after the incumbent left. The staff size of the department change from 38 to 25 .
} 


\begin{tabular}{|c|c|c|c|c|}
\hline & \multicolumn{2}{|c|}{ First Measurement } & \multicolumn{2}{|c|}{ Second Measurement } \\
\hline & $\mathbf{N}$ & $\%$ & $\mathbf{N}$ & $\%$ \\
\hline \multicolumn{5}{|l|}{ Sex } \\
\hline Female & 88 & $66.2 \%$ & 73 & $58.4 \%$ \\
\hline Male & 19 & $14.3 \%$ & 25 & $20.0 \%$ \\
\hline Prefer not to respond & 26 & $19.5 \%$ & 27 & $21.6 \%$ \\
\hline \multicolumn{5}{|l|}{ Age } \\
\hline Under 20 & 1 & $0.8 \%$ & 2 & $1.6 \%$ \\
\hline $20-29$ & 28 & $21.1 \%$ & 8 & $6.4 \%$ \\
\hline $30-39$ & 22 & $16.5 \%$ & 15 & $12.0 \%$ \\
\hline $40-49$ & 29 & $21.8 \%$ & 25 & $20.0 \%$ \\
\hline $50-59$ & 23 & $17.3 \%$ & 20 & $16.0 \%$ \\
\hline 60 or over & 6 & $4.5 \%$ & 5 & $4.0 \%$ \\
\hline Prefer not to respond & 24 & $18.0 \%$ & 50 & $40.0 \%$ \\
\hline \multicolumn{5}{|l|}{ Ethnic Background } \\
\hline Asian & 3 & $2.3 \%$ & 1 & $0.8 \%$ \\
\hline Black or African American & 10 & $7.5 \%$ & 9 & $7.2 \%$ \\
\hline Hispanic & 69 & $51.9 \%$ & 69 & $55.2 \%$ \\
\hline White/Caucasian & 17 & $12.8 \%$ & 14 & $11.2 \%$ \\
\hline Other & 5 & $3.8 \%$ & 3 & $2.4 \%$ \\
\hline Prefer not to respond & 29 & $21.8 \%$ & 29 & $23.2 \%$ \\
\hline \multicolumn{5}{|l|}{ Education } \\
\hline High School & 10 & $7.5 \%$ & 8 & $6.4 \%$ \\
\hline Some College & 33 & $24.8 \%$ & 31 & $24.8 \%$ \\
\hline Associate's/Technical degree & 7 & $5.3 \%$ & 11 & $8.8 \%$ \\
\hline Bachelor's degree & 28 & $21.1 \%$ & 28 & $22.4 \%$ \\
\hline Some graduate work & 13 & $9.8 \%$ & 7 & $5.6 \%$ \\
\hline Master's degree & 16 & $12.0 \%$ & 13 & $10.4 \%$ \\
\hline Doctoral degree & 0 & $0.0 \%$ & 1 & $0.8 \%$ \\
\hline Other & 0 & $0.0 \%$ & 0 & $0.0 \%$ \\
\hline Prefer not to respond & 26 & $19.5 \%$ & 26 & $20.8 \%$ \\
\hline \multicolumn{5}{|l|}{ Organization Level } \\
\hline Non-Management & 66 & $49.6 \%$ & 62 & $49.6 \%$ \\
\hline Line Management & 27 & $20.3 \%$ & 19 & $15.2 \%$ \\
\hline Middle Management & 10 & $7.5 \%$ & 7 & $5.6 \%$ \\
\hline Senior Management & 0 & $0.0 \%$ & 0 & $0.0 \%$ \\
\hline Prefer not to respond & 30 & $22.6 \%$ & 37 & $29.6 \%$ \\
\hline \multicolumn{5}{|l|}{ Salary } \\
\hline$\$ 18,000$ or less & 4 & $3.0 \%$ & 9 & $7.2 \%$ \\
\hline$\$ 18,001$ to $\$ 25,000$ & 23 & $17.3 \%$ & 27 & $21.6 \%$ \\
\hline$\$ 25,001$ to $\$ 35,000$ & 42 & $31.6 \%$ & 36 & $28.8 \%$ \\
\hline$\$ 35,001$ to $\$ 45,000$ & 16 & $12.0 \%$ & 13 & $10.4 \%$ \\
\hline$\$ 45,001$ to $\$ 60,000$ & 12 & $9.0 \%$ & 8 & $6.4 \%$ \\
\hline$\$ 60,001$ to $\$ 75,000$ & 5 & $3.8 \%$ & 3 & $2.4 \%$ \\
\hline$\$ 75,001$ to $\$ 90,000$ & 0 & $0.0 \%$ & 0 & $0.0 \%$ \\
\hline$\$ 90,001$ plus & 1 & $0.8 \%$ & 1 & $0.8 \%$ \\
\hline Prefer not to respond & 30 & $22.6 \%$ & 28 & $22.4 \%$ \\
\hline
\end{tabular}




\section{Years with Organization}

Less than 6 months

6 months to 1 year

1 to 2 years

2 to 4 years

4 to 6 years

6 to 10 years

10 to 15 years

More than 15 years

Prefer not to respond

\begin{tabular}{rrrr}
9 & $6.8 \%$ & 9 & $7.2 \%$ \\
9 & $6.8 \%$ & 6 & $4.8 \%$ \\
16 & $12.0 \%$ & 13 & $10.4 \%$ \\
10 & $7.5 \%$ & 14 & $11.2 \%$ \\
13 & $9.8 \%$ & 11 & $8.8 \%$ \\
22 & $16.5 \%$ & 18 & $14.4 \%$ \\
11 & $8.3 \%$ & 12 & $9.6 \%$ \\
20 & $15.0 \%$ & 16 & $12.8 \%$ \\
23 & $17.3 \%$ & 26 & $20.8 \%$ \\
\hline
\end{tabular}

Table 3 - Demographics of survey participants

\begin{tabular}{|l|l|l|}
\hline Department & $\begin{array}{l}\text { First } \\
\text { Measurement }\end{array}$ & $\begin{array}{l}\text { Second } \\
\text { Measurement }\end{array}$ \\
\hline $\begin{array}{l}\text { Student } \\
\text { Administration }\end{array}$ & 97 & 111 \\
\hline $\begin{array}{l}\text { Controller's } \\
\text { Office }\end{array}$ & 36 & 24 \\
\hline Total & $\mathbf{1 3 3}$ & $\mathbf{1 2 5}$ \\
\hline
\end{tabular}

Table 4 - Participation by department

The research employed multivariate and path analysis methods to examine the variables for change and corresponding relationships. This contingency approach allowed for the separation of variables to focus on single characteristic relationships. Individual variables were tested in single regression models on each independent and dependent combination. Then the survey items were grouped into the information system environment characteristics, job design characteristics, and the operating culture dimensions and tested by multivariate procedures.

The unit of measure was a group (represented by the entire sample) for the factor, multivariate, and path analysis. The path analysis technique was selected to test potential determinants of the effects and contributions to the effects made by each variable within 
the given environment. The responses were also paired as a set for an individual's before and after perception of each of the variables in a formal matched pair analysis. This unit of measure was the individual. Differences between the old and new system characteristics are reflected in the perception of an individual's job design characteristics and the organizational culture's dimension measurements.

The analysis of the data will be presented in the next chapter. 
V. Data Analysis

Three datasets were created from the data: responses to the surveys while using the legacy system labeled first measurement, responses during the use of the ERP system labeled second measurement, and the third was of the result of the difference between the first and second measurement (first response - second response). Each dataset was analyzed separately to support two types of procedures: multivariate regression procedures including path analysis and step-wise regression, and matched pair analysis. The multivariate procedures included data cleaning, principal component analysis, path regression, and step-wise regression. All survey items by instrument are used as input to the principal component analysis to reduce the number of variables to only significant factors. Reliability of the factors is tested before running the multiple path and step-wise regressions. The matched pair analysis used the first and second measurement datasets, and each variable is based on the set of items being summed and then averaged by individual. The reliability of the variables is detailed in the matched-pair analysis summary. This data analysis section is organized by measurement period, question, and type of statistical procedure. Interpretation of the findings is contained in the discussion chapter.

First Measurement

The first survey response period took place during the months of May and June of 2004 and had an overall participation rate of $99 \%$. Participants were asked to evaluate the legacy computer system, their current job, and the current operating environment. The survey was administered prior to the departments receiving training on the new system. 
As stated before, 133 response cases were collected in the first measurement and 130 cases were retained for analysis. The Mahalanobis distance calculation was performed using all items and all remaining cases. The test statistic was chi-square with $\mathrm{df}=100+$ of 153.2 . No values were above 97 ; therefore, no multivariate outliers were detected. A visual inspection of the data was performed on the 157 individual survey items. Eighteen variables were transformed due to extreme skewness $(>1.0)$ in an effort to increase the strength of the variables.

The original items were explored in both a univariate and a multivariate analysis. In the univariate analysis, to combine the items for the given variable, summed totals of the items are used. Items that required reversed scoring were translated before summing.

Factor Analysis

After examining the data for logical patterns, a factor analysis was conducted to determine an underlying structure for all of the variables and to reduce the number of items used in the analysis to a small set of factors. Principal component analysis was conducted utilizing a varimax rotation. The initial analysis retained 22 components. Four criteria were used to determine the appropriate number of components to retain: eigenvalue, variance, scree plot, and residuals. These criteria indicated retaining five components. Thus, principal components analysis was conducted to retain five components and apply the varimax rotation.

After rotation, the five components accounted for $64 \%$ of the explained variance. All component loadings were positive. The variance explained and reliability score 
(Cronbach's Alpha) by the components is detailed in Table 5. (Components 4 and 5 loaded only one item and the ${ }^{* * *}$ indicate value less than .50 ).

\begin{tabular}{|c|c|c|c|}
\hline Component Loadings & $\begin{array}{l}\text { Variance } \\
\text { Explained }\end{array}$ & $\frac{\text { Cronbach's }}{\text { Alpha }}$ & Items loaded description \\
\hline 1 - System Characteristics & $31 \%$ & 0.93 & Detail, location, ease of use, meaning \\
\hline 2 - Compat/Confusion & $12 \%$ & 0.76 & Compatibility and positive confusion \\
\hline 3- System Accuracy & $9 \%$ & 0.6 & Data accuracy \\
\hline 4 - System Reliability & $6 \%$ & $* * *$ & System available when needed \\
\hline 5 - System Perf & $6 \%$ & $* * *$ & System performing as needed \\
\hline
\end{tabular}

Table 5 - TTF component loadings (***-refers to values less than .5)

The first TTF component, labeled system characteristics, consists of items relating to general system characteristics such as the level of data detail, the location of the data in the system, the ease of using the system, and the data meaning represented in the system. The second component accounted for the level of system compatibility with the work tasks and the level of discrepancies (or confusion) found when comparing the system data to other systems with similar data, and is labeled compat/confusion. The third component is labeled data accuracy and reflects the level of correctness found within the system. The fourth and fifth components reflect system operations as seen by the user.

The JDS instrument consists of two sections containing a total of twenty-one questions defining seven constructs. They are the job autonomy, job identity, job variety, job significance, feedback from job, feedback from supervisor, and work with others. The original items were explored in both a univariate and a multivariate analysis. In the univariate analysis, to combine the items for the given variable, summed totals of the items are used. Items that required reversed scoring were translated before summing. The mean and standard deviation of the summed items can be found in Table 6. 
Std.

\begin{tabular}{|cccc|}
\hline Survey & Variables & Mean & Deviation \\
& Level of Detail & 4.51 & 1.30 \\
& Accuracy & 4.21 & 1.40 \\
& Compatibility & 4.51 & 1.23 \\
& Location & 3.83 & 1.34 \\
Technology & Accessibility & 4.62 & 1.55 \\
Task Fit & Meaning & 4.35 & 1.56 \\
& Assistance & 5.07 & 1.52 \\
& Ease of Use & 4.73 & 1.17 \\
& System Reliability & 4.90 & 1.26 \\
& Currency & 3.40 & 1.69 \\
& Presentation & 4.91 & 1.47 \\
& Confusion & 4.00 & 1.60 \\
\hline Job & Variety & 4.59 & 0.85 \\
Diagnostic & Identity & 4.75 & 0.88 \\
Survey & Autonomy & 5.10 & 0.86 \\
& Feedback & 4.90 & 0.81 \\
& Significance & 4.63 & 0.86 \\
& Work with Others & 5.10 & 0.86 \\
\hline Inventory & Achievement & 33.91 & 7.54 \\
& SelfAct & 31.53 & 8.40 \\
& Dependent & 35.35 & 6.89 \\
& Avoid & 24.12 & 8.29 \\
\hline & Oppositional & 24.06 & 6.32 \\
& Power & 26.95 & 9.17 \\
& Competitive & 22.65 & 7.18 \\
& Perfect & 33.24 & 7.54 \\
\hline
\end{tabular}

Table 6 - Means and standard deviation of summed measurements from first measurement.

The original research model proposes a positive relationship between all but one of the TTF variables with the JDS variables. The outstanding TTF variable is confusion. It has a negative relationship with the JDS variables. The Pearson factor and significance level found in the Table 7 confirms the significant positive relationships (.01) for all of the TTF variables with the exception of reliability, compatibility, and ease of use 
(significant at the .10 level), and the negative confusion variable (significant at the .10 level) of one or more of the JDS variables. The only JDS variable not significantly correlated with any TTF variable is autonomy.

\begin{tabular}{|c|c|c|c|c|c|c|}
\hline & \multicolumn{6}{|c|}{ Job Diagnostic Survey (JDS) } \\
\hline & Variety & Identity & Significance & Autonomy & Feedback & Others \\
\hline Ease of Use & 0.169 & 0.19 & -0.052 & -0.065 & 0.094 & -0.126 \\
\hline p-value & 0.028 & 0.015 & 0.278 & 0.23 & 0.143 & 0.077 \\
\hline Location & 0.213 & 0.279 & 0.206 & 0.026 & 0.14 & 0.032 \\
\hline$p$-value & 0.008 & 0.001 & 0.01 & 0.386 & 0.057 & 0.36 \\
\hline Compat & -0.019 & -0.06 & -0.121 & -0.024 & -0.008 & -0.135 \\
\hline p-value & 0.414 & 0.25 & 0.087 & 0.395 & 0.466 & 0.064 \\
\hline Accuracy & 0.187 & 0.118 & -0.004 & 0.018 & 0.001 & 0.097 \\
\hline p-value & 0.018 & 0.091 & 0.48 & 0.418 & 0.496 & 0.138 \\
\hline Currency & -0.225 & -0.287 & -0.169 & -0.035 & -0.071 & 0.035 \\
\hline$p$-value & 0.005 & 0.00 & 0.027 & 0.345 & 0.213 & 0.347 \\
\hline Access & 0.236 & 0.287 & 0.157 & 0.03 & 0.11 & 0.024 \\
\hline$p$-value & 0.004 & 0.001 & 0.038 & 0.369 & 0.107 & 0.392 \\
\hline Confusion & -0.154 & -0.108 & -0.006 & -0.01 & -0.119 & -0.016 \\
\hline p-value & 0.04 & 0.111 & 0.471 & 0.457 & 0.089 & 0.427 \\
\hline Help & 0.16 & 0.22 & 0.08 & 0.126 & 0.126 & 0.018 \\
\hline$p$-value & 0.035 & 0.006 & 0.182 & 0.077 & 0.077 & 0.419 \\
\hline Detail & 0.196 & 0.316 & 0.151 & 0.079 & 0.123 & 0.08 \\
\hline p-value & 0.013 & 0.00 & 0.044 & 0.187 & 0.083 & 0.183 \\
\hline Meaning & 0.109 & 0.26 & 0.025 & -0.029 & 0.078 & -0.053 \\
\hline$p$-value & 0.111 & 0.002 & 0.391 & 0.372 & 0.189 & 0.277 \\
\hline Presentation & 0.124 & 0.218 & 0.032 & 0.076 & 0.204 & 0.097 \\
\hline p-value & 0.081 & 0.007 & 0.358 & 0.195 & 0.01 & 0.136 \\
\hline Reliability & 0.113 & 0.048 & -0.004 & -0.038 & 0.042 & -0.042 \\
\hline p-value & 0.1 & 0.295 & 0.482 & 0.334 & 0.316 & 0.317 \\
\hline
\end{tabular}

Table 7 - Pearson Correlations of Technology Task Fit survey (TTF) and the Job Diagnostic Survey (JDS) for the first measurement

After examining the data for logical patterns, a factor analysis was conducted to determine an underlying structure for all items. The items were treated as single variables and not combined with other like constructs. This type of analysis reduces the number of items used in the analysis to a small set of factors. A principal component analysis was 
conducted utilizing a varimax rotation. The initial analysis retained five components.

Four criteria were used to determine the appropriate number of components to retain: eigenvalue, variance, scree plot, and residuals. Criteria indicated retaining four components. Thus, a principal components analysis was conducted to retain four components and apply the varimax rotation.

After rotation, the four components accounted for $68 \%$ of the explained variance. All component loadings were positive. The variance explained and reliability score (Cronbach's Alpha) by the components is detailed in Table 8 . The overall reliability score of the JDS instrument with this data set is .84 .

\begin{tabular}{|c|c|c|c|}
\hline & Variance & Cronbach's & \\
\hline Component Loadings & Explained & Alpha & Items loaded description \\
\hline 1 - Feedback from Job & $20 \%$ & 0.83 & Job signals for correct behavior \\
\hline 2 - Job Variety & $18 \%$ & 0.78 & Variety of work tasks \\
\hline 3-Job Autonomy & $17 \%$ & 0.75 & Own decision of work \\
\hline 4-Job Significance & $12 \%$ & 0.58 & Job importance \\
\hline
\end{tabular}

Table 8 - JDS component loadings

The first JDS component consists of all items relating to feedback from the job. This is the extent to which the job provides information about the work performance. In this environment, this is the strongest job characteristic. This indicates that workers judge their performance through feedback and interactions on the job. The second component reflects how many different skills and activities are required by the job. The third component of autonomy describes how much the individual has the ability to decide how to work. The last component of significance is the perceived importance of the job or the results of the job in affecting other people. 
The factor loadings were also tested for significant correlations and the results appear in Table 9. Only the TTF's information system characteristics factor was correlated significantly (at the .01 level) with the two JDS factors of feedback and significance. All of the TTF factors are correlated with one or more of the JDS factors at the higher significance level (.10).

\begin{tabular}{|c|c|c|c|c|c|}
\hline & & Job Diagn & $\begin{array}{c}\text { tic Survey } \\
2\end{array}$ & Factors & \\
\hline & & 1 Feedback & Variety & 3 Autonomy & 4 Significance \\
\hline & 1 Characteristics & 0.32 & -0.02 & 0.16 & 0.32 \\
\hline & p-value & 0.00 & 0.40 & 0.04 & 0.00 \\
\hline & $\begin{array}{l}2 \text { Compat/ } \\
\text { Confusion }\end{array}$ & 0.09 & -0.03 & 0.01 & -0.13 \\
\hline Technology & $p$-value & 0.16 & 0.36 & 0.46 & 0.08 \\
\hline $\begin{array}{l}\text { Task Fit } \\
\text { Survey }\end{array}$ & 3 Accuracy & -0.13 & -0.17 & -0.01 & 0.06 \\
\hline Factors & $p$-value & 0.08 & 0.03 & 0.47 & 0.25 \\
\hline & 4 Reliability & -0.01 & 0.13 & 0.08 & 0.02 \\
\hline & $p$-value & 0.47 & 0.08 & 0.21 & 0.41 \\
\hline & 5 Performance & -0.06 & -0.13 & 0.12 & -0.01 \\
\hline & $p$-value & 0.26 & 0.08 & 0.09 & 0.46 \\
\hline
\end{tabular}

Table 9 - Factor Correlations for TTF survey and JDS survey

The OCI instrument consists of 120 single items grouped into 12 constructs and three groupings of the constructs. The original items were explored in both a univariate and a multivariate analysis. In the univariate analysis, to combine the items for the given variable, summed totals of the items by the 12 constructs are used.

The correlation between the JDS and OCI variables had been established in prior studies (Cooke and Szumal, 2000). The correlations found in the first measurement differed from published relationships. The signs of the correlations were correctly predicted; however, not all correlations are significant as established in previous studies. This may be due to the smaller population size of this study. The correlation values for the JDS and OCI items are reported in Table 10. 
Job Diagnostic Survey (JDS)

\begin{tabular}{|c|c|c|c|c|c|c|}
\hline & Variety & Identity & Significance & Autonomy & Feedback & $\begin{array}{c}\text { Work } \\
\text { w/Others }\end{array}$ \\
\hline \multirow{2}{*}{$\begin{array}{l}\text { ACHIEVE } \\
\text { p-value }\end{array}$} & 0.19 & 0.17 & 0.01 & -0.01 & 0.19 & 0.12 \\
\hline & 0.02 & 0.03 & 0.45 & 0.48 & 0.02 & 0.10 \\
\hline \multirow{2}{*}{$\begin{array}{l}\text { SELFACT } \\
\text { p-value } \\
\end{array}$} & 0.08 & 0.21 & 0.06 & 0.02 & 0.12 & 0.06 \\
\hline & 0.21 & 0.01 & 0.27 & 0.44 & 0.10 & 0.28 \\
\hline \multirow{2}{*}{$\begin{array}{l}\text { HUMAN } \\
\text { p-value }\end{array}$} & 0.21 & 0.30 & 0.06 & 0.07 & 0.16 & 0.03 \\
\hline & 0.01 & 0.00 & 0.27 & 0.23 & 0.04 & 0.37 \\
\hline \multirow{2}{*}{$\begin{array}{r}\text { AFFLIATIVE } \\
p \text {-value } \\
\end{array}$} & 0.11 & 0.25 & 0.00 & -0.01 & 0.18 & 0.10 \\
\hline & 0.12 & 0.00 & 0.49 & 0.46 & 0.02 & 0.15 \\
\hline \multirow{2}{*}{$\begin{array}{r}\text { APPROVAL } \\
p \text {-value } \\
\end{array}$} & 0.09 & 0.10 & 0.08 & 0.18 & 0.09 & 0.12 \\
\hline & 0.16 & 0.14 & 0.21 & 0.02 & 0.16 & 0.10 \\
\hline \multirow{2}{*}{$\begin{array}{l}\text { CONVENT } \\
p \text {-value }\end{array}$} & -0.05 & -0.11 & 0.09 & 0.02 & 0.02 & 0.05 \\
\hline & 0.30 & 0.12 & 0.16 & 0.44 & 0.42 & 0.30 \\
\hline \multirow{2}{*}{$\begin{array}{r}\text { DEPENDENT } \\
p \text {-value }\end{array}$} & 0.04 & -0.08 & 0.12 & 0.18 & -0.12 & -0.01 \\
\hline & 0.33 & 0.20 & 0.10 & 0.03 & 0.09 & 0.46 \\
\hline \multirow{2}{*}{$\begin{array}{l}\text { AVOID } \\
\text { p-value }\end{array}$} & -0.02 & -0.13 & 0.02 & 0.16 & -0.14 & -0.04 \\
\hline & 0.42 & 0.09 & 0.44 & 0.04 & 0.07 & 0.33 \\
\hline \multirow{2}{*}{$\begin{array}{l}\text { OPPOSIT } \\
p \text {-value }\end{array}$} & 0.02 & 0.00 & 0.19 & 0.03 & -0.04 & -0.04 \\
\hline & 0.40 & 0.49 & 0.02 & 0.39 & 0.32 & 0.32 \\
\hline \multirow{2}{*}{$\begin{array}{l}\text { POWER } \\
\qquad \text {-value }\end{array}$} & 0.02 & -0.19 & 0.00 & -0.01 & -0.09 & 0.00 \\
\hline & 0.43 & 0.02 & 0.50 & 0.44 & 0.18 & 0.49 \\
\hline \multirow{2}{*}{$\begin{array}{r}\text { COMPETITIVE } \\
p \text {-value }\end{array}$} & 0.07 & -0.06 & 0.00 & 0.04 & 0.00 & 0.09 \\
\hline & 0.23 & 0.26 & 0.49 & 0.33 & 0.48 & 0.18 \\
\hline \multirow{2}{*}{$\begin{array}{r}\text { PERFECTION } \\
p \text {-value } \\
\end{array}$} & -0.03 & -0.13 & 0.12 & -0.10 & 0.11 & 0.09 \\
\hline & 0.38 & 0.09 & 0.10 & 0.14 & 0.12 & 0.17 \\
\hline
\end{tabular}

Table 10 - Job Diagnostic Survey and Organization Culture Inventory correlations for the first measurement

The initial factor analysis uses the raw 120 items as input. The first data reduction

returned 30 factors with eigen values above 1.0. This factoring solution revealed half of the variance explained by the first seven components and the total variance explained in 103 items. The 30 factors only clustered 56 items. In an effort to reduce the number of variables, several factor models were tested. The best raw data model loaded all of the items into five forced factors for a $46.58 \%$ variance explanation. The reliability of each component is listed in Table 11. 


\begin{tabular}{|c|c|c|c|}
\hline Component Loadings & $\begin{array}{l}\text { Variance } \\
\text { Explained }\end{array}$ & $\begin{array}{l}\text { Cronbach's } \\
\text { Alpha }\end{array}$ & Items loaded description \\
\hline 1 - Constructive & $22.21 \%$ & 0.93 & Collection of all constructive items \\
\hline 2-Competitive & $12.39 \%$ & 0.85 & Competitive \\
\hline 3 - Avoidance & $5.17 \%$ & 0.85 & Avoidance \\
\hline 4-Perfect & $3.90 \%$ & 0.78 & Perfect \\
\hline 5-Approval & $2.89 \%$ & 0.74 & Approval \\
\hline
\end{tabular}

Table 11 - Organization Culture Inventory Components Loadings

The first component is made up of 42 items from the constructive dimensions of achievement, self-actualizing, humanistic-encouraging, and affiliative and is named constructive. The second and fourth components represent the aggressive/defensive group. The second has nine items from the competitive dimension and is labeled competitive. The fourth is named for perfection due to the nine items that loaded in this factor. The third and fifth components come from the passive/defensive group. The third component has 10 items from avoidance and is labeled avoidance. The fifth component contains three items from the approval dimension and is labeled approval.

The factors were compared to each other in a correlation analysis. The highest correlation between the OCI and JDS factors is between the feedback and constructive factors at .42 with significance of .000 . The results appear in Table 12.

Path Analysis

Path analysis regressions were conducted to determine the causal effects among the system characteristics, job factors, and OCI factors during the first measurement period. Only five paths were significant ( $p<.10$ level). Three of them were between the TTF factor of system characteristics and the JDS factors of feedback (beta $=.325, p=$ $.000)$, autonomy (beta $=.152, p=.098)$, and significance $($ beta $=.329, p=.000)$. 


\begin{tabular}{|c|c|c|c|c|c|c|}
\hline & & \multicolumn{5}{|c|}{ Organization Culture Inventory Factors } \\
\hline & & Constructive & Competitive & Avoidance & Perfection & Approval \\
\hline \multirow{8}{*}{$\begin{array}{c}\text { Job } \\
\text { Diagnostic } \\
\text { Survey }\end{array}$} & Feedback & 0.42 & 0.13 & -0.16 & -0.11 & -0.03 \\
\hline & p-value & 0.00 & 0.09 & 0.06 & 0.15 & 0.39 \\
\hline & Variety & -0.10 & 0.09 & -0.11 & -0.06 & 0.07 \\
\hline & p-value & 0.15 & 0.19 & 0.13 & 0.28 & 0.25 \\
\hline & Autonomy & 0.18 & -0.16 & 0.12 & -0.19 & 0.15 \\
\hline & p-value & 0.04 & 0.06 & 0.12 & 0.03 & 0.07 \\
\hline & Significance & 0.03 & -0.11 & -0.02 & 0.06 & 0.09 \\
\hline & $p$-value & 0.37 & 0.15 & 0.44 & 0.27 & 0.19 \\
\hline \multirow{10}{*}{$\begin{array}{c}\text { Technology } \\
\text { Task Fit } \\
\text { Survey }\end{array}$} & Characteristics & 0.40 & 0.05 & -0.13 & -0.13 & 0.09 \\
\hline & p-value & 0.00 & 0.31 & 0.10 & 0.11 & 0.20 \\
\hline & Confusion & 0.16 & 0.45 & -0.01 & 0.11 & -0.12 \\
\hline & p-value & 0.06 & 0.00 & 0.45 & 0.15 & 0.12 \\
\hline & Accuracy & -0.05 & 0.04 & 0.17 & 0.06 & -0.08 \\
\hline & p-value & 0.31 & 0.34 & 0.05 & 0.29 & 0.22 \\
\hline & Reliability & 0.00 & -0.20 & -0.31 & -0.13 & 0.10 \\
\hline & p-value & 0.49 & 0.02 & 0.00 & 0.10 & 0.15 \\
\hline & Performance & 0.06 & -0.05 & 0.06 & -0.02 & 0.05 \\
\hline & p-value & 0.28 & 0.30 & 0.29 & 0.44 & 0.33 \\
\hline
\end{tabular}

Table 12 - Job Diagnostic Survey and Technology Task Fit survey with the Organization Culture Inventory Factor Correlations for the first measurement

The fourth path was a negative relationship between system accuracy and feedback (beta $=-.167, p=.068)$. The only significant path to the OCI factors was between feedback and constructive (beta $=.427, p=.000$ ). Several paths to the OCI constructs were initially proposed but had to be dismissed due to inconsistencies between the empirical and reproduced correlations. (The coefficients and p-values of the paths can be seen in Figure 5.) 


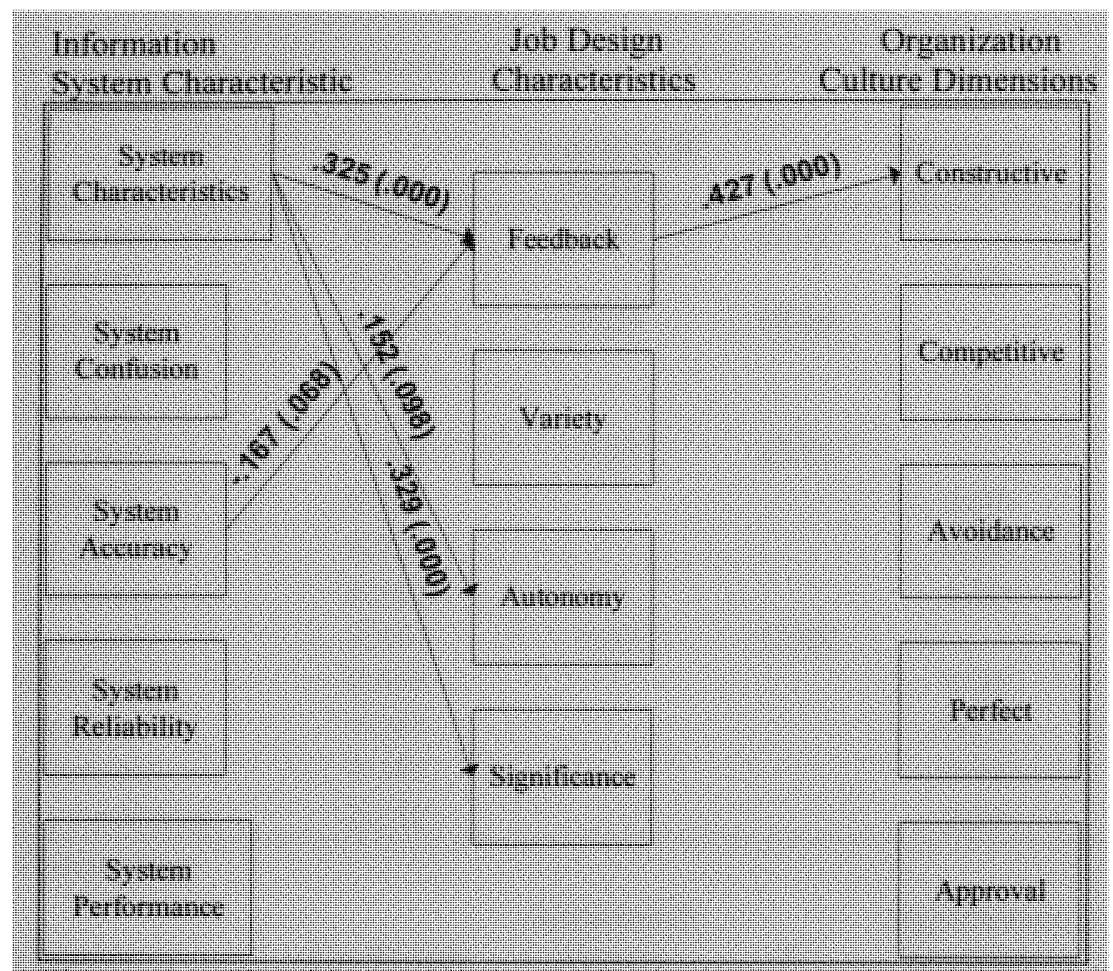

Figure 5-First Measurement Path Analysis

Step-wise Regression

To further understand the correlation among several variables, a regression analysis is used to eliminate conjoint correlations and eliminate significant, but trivial, correlations. A step-wise regression method was used to return only the factors that have a significant relationship with the dependent variable. All of the factors were loaded with the dependent variable of each of the OCI factors. Only three of the OCI factors had significant relationships with the combinations. The results appear in Table 13. 


\begin{tabular}{|c|c|c|c|c|c|c|}
\hline \multicolumn{7}{|c|}{ Factor 1: Constructive } \\
\hline Model & Variable & Coefficient & $\begin{array}{c}\text { Adjusted R } \\
\text { Square }\end{array}$ & $\mathbf{F}$ & $\begin{array}{c}\text { Significance } \\
(p \text {-value })\end{array}$ & VIF \\
\hline \multirow[t]{2}{*}{1} & (Constant) & -0.009 & 0.20 & 25.15 & & \\
\hline & Feedback & 0.476 & & & 0.00 & 1.00 \\
\hline \multirow[t]{3}{*}{2} & (Constant) & -0.042 & 0.27 & 19.00 & & \\
\hline & Feedback & 0.443 & & & 0.00 & 1.09 \\
\hline & Sys Char & 0.258 & & & 0.00 & 1.09 \\
\hline \multirow[t]{4}{*}{3} & (Constant) & -0.045 & 0.31 & 15.71 & & \\
\hline & Feedback & 0.466 & & & 0.00 & 1.11 \\
\hline & Sys Char & 0.250 & & & 0.00 & 1.09 \\
\hline & Autonomy & 0.167 & & & 0.00 & 1.02 \\
\hline \multirow[t]{5}{*}{4} & (Constant) & -0.051 & 0.33 & 12.80 & & \\
\hline & Feedback & 0.449 & & & 0.00 & 1.12 \\
\hline & Sys Char & 0.264 & & & 0.00 & 1.10 \\
\hline & Autonomy & 0.167 & & & 0.00 & 1.02 \\
\hline & Sys Confusion & 0.159 & & & 0.00 & 1.01 \\
\hline \multicolumn{7}{|c|}{ Factor 2: Competitive } \\
\hline Model & Variable & Coefficient & $\begin{array}{c}\text { Adjusted R } \\
\text { Square } \\
\end{array}$ & $\mathbf{F}$ & $\begin{array}{c}\text { Significance } \\
(p \text {-value })\end{array}$ & VIF \\
\hline \multirow[t]{2}{*}{1} & (Constant) & 0.020 & 0.20 & 25.74 & & \\
\hline & Sys Confusion & 0.110 & & & 0.000 & 1.00 \\
\hline \multirow[t]{3}{*}{2} & (Constant) & 0.101 & 0.22 & 14.71 & & \\
\hline & Sys Confusion & 0.097 & & & 0.000 & 1.01 \\
\hline & Sys Reliability & -0.145 & & & 0.000 & 1.01 \\
\hline \multicolumn{7}{|c|}{ Factor 3: Avoidance } \\
\hline Model & Variable & Coefficient & $\begin{array}{c}\text { Adjusted R } \\
\text { Square } \\
\end{array}$ & $\mathbf{F}$ & $\begin{array}{c}\text { Significance } \\
(p \text {-value })\end{array}$ & VIF \\
\hline \multirow[t]{2}{*}{1} & (Constant) & -0.003 & 0.100 & 10.34 & & \\
\hline & Sys Reliability & -0.075 & & & 0.000 & 1.00 \\
\hline \multirow[t]{3}{*}{2} & (Constant) & 0.006 & 0.140 & 7.68 & & \\
\hline & Sys Reliability & -0.070 & & & 0.000 & 1.03 \\
\hline & Autonomy & -0.004 & & & 0.000 & 1.03 \\
\hline \multirow[t]{4}{*}{3} & (Constant) & 0.007 & 0.190 & 7.27 & & \\
\hline & Sys Reliability & -0.093 & & & 0.000 & 1.04 \\
\hline & Autonomy & 0.011 & & & 0.000 & 1.03 \\
\hline & Sys Accuracy & -0.093 & & & 0.000 & 1.02 \\
\hline
\end{tabular}

Table 13 - First Measurement Regression Models

Regression results indicate an overall model of four predictors (feedback, system characteristics, autonomy, and system confusion) for the OCI factor of constructive. Two other OCI factors, competitive and avoidance had significant models. Each of the models 
do not account for a large amount of variance (represented by adjusted $\mathrm{R}^{2}$ ). The low $\mathrm{R}^{2}$ value on the information system factors is expected due to previously published studies using the same instrument. This issue of these low values will be discussed later. The results do indicate that a direct relationship of information system characteristics combined with job design characteristics exists with the organizational culture dimensions.

Second Measurement

The second survey response period took place during November and December of 2004. One hundred and twenty four responses were collected from four university departments for an overall participation rate of $99 \%$. Participants were asked to evaluate the new ERP system (PantherSoft), their current job, and the current operating environment. PantherSoft had replaced many of the legacy systems and was the primary computer system used by the departments in the survey.

Of the collected responses, 114 cases were retained in the first measurement after 10 cases were dropped due to lack of valid data. Missing data for the measurements ranged from $0-2 \%$ per item, and all variables were retained in the analysis. The Mahalanobis distance calculation was performed using all items and all remaining cases. No multivariate outliers were detected. A visual inspection of the data was performed on the 157 individual survey items. Twelve variables were transformed due to extreme skewness $(>1.0)$ in an effort to increase the strength of the variables. Two participants answered the surveys with the same responses for each item (unique to each survey). This may be due to trying to complete the survey in a rush and ignoring the questions. The 
responses were originally kept but may be deleted due to outlier behavior later in the univariate analysis.

As in the first measurement, each of the instrument summed variables is examined for correlations. All variables are calculated the same as in the first measurement. The items were explored in both a univariate and a multivariate analysis. In the univariate analysis, to combine the items for the given variable, summed totals of the items are used. Items that required reversed scoring were translated before summing. Means and standard deviations for the second measurement are located in Table 14.

Factor Analysis

After the TTF data was investigated for logical patterns, a factor analysis was conducted to determine an underlying structure for all of the variables and reduce the number of items used in the analysis to a small set of factors. A principal component analysis was conducted utilizing a varimax rotation. The initial analysis retained five components. Four criteria were used to determine the appropriate number of components to retain: eigenvalue, variance, scree plot, and residuals. Criteria indicated retaining three components. Thus, a principal components analysis was conducted to retain three components and apply the varimax rotation.

After rotation, the three components accounted for $62 \%$ of the explained variance. All component loadings were positive. The variance explained and reliability score (Cronbach's Alpha) by the components are detailed in Table 15. The overall reliability score for all loaded TTF items is .904 . 
Std.

\begin{tabular}{|llll|}
\hline Survey & Variables & Mean & Deviation \\
\hline & Level of Detail & 4.51 & 1.51 \\
& Accuracy & 4.00 & 1.16 \\
& Compatibility & 4.72 & 1.38 \\
& Location & 3.56 & 1.33 \\
& Accessibility & 4.37 & 1.42 \\
Technology & Meaning & 4.23 & 1.61 \\
Task Fit & Assistance & 4.49 & 1.37 \\
& Ease of Use & 4.70 & 1.06 \\
& System & & \\
& Reliability & 4.25 & 1.18 \\
& Currency & 3.76 & 1.56 \\
& Presentation & 4.41 & 1.51 \\
& Confusion & 4.50 & 1.39 \\
\hline Job & Variety & 4.219 & 1.278 \\
Diagnostic & Identity & 4.289 & 1.288 \\
Survey & Autonomy & 4.054 & 1.356 \\
& Feedback & 4.396 & 1.352 \\
& Significance & 5.55 & 0.998 \\
& Work with & & \\
Inventory & Work w/Others & 5.062 & 1.168 \\
\hline & Perfect & 33.12 & 7.871 \\
\hline & Achievement & 33.667 & 7.54 \\
& SelfAct & 31.361 & 8.815 \\
& Humanistic & 32.852 & 9.143 \\
& Affliative & 36.204 & 9.502 \\
\hline & Approval & 29.11 & 8.368 \\
& Convent & 30.944 & 7.603 \\
& Dependent & 31.806 & 7.905 \\
& Cower & 23.713 & 8.395 \\
\hline
\end{tabular}

Table 14 - Means and standard deviation of summed measurements from second measurement.

\begin{tabular}{|c|c|c|c|}
\hline & Variance & Cronbach's & \\
\hline Component Loadings & Explained & Alpha & Items loaded description \\
\hline 1 - Look and Work & $24.33 \%$ & 0.880 & Ease, presentation, access, meaning \\
\hline 2 - Compatibility & $19.17 \%$ & 0.749 & Compatibility/currency \\
\hline 3-Detail/Accuracy & $18.50 \%$ & 0.681 & Level of detail/accuracy \\
\hline
\end{tabular}

Table 15 -TTF Components - second measurement 
The first TTF component is made up of items relating to the presentation and ease of working with the system such as the ease of use, presentation, access, and data meaning. The second component accounts for the level of system compatibility with the work tasks and the currency of the data. The third component reflects the data detail and accuracy within the system.

As in the first measurement, a univariate analysis is conducted on the JDS variables. To combine the items for the given variable, summed totals of the items are used. Items that required reversed scoring were translated before summing. The mean and standard deviation of the summed items can be found in the earlier section of TTF Table 13.

The JDS data was also examined for logical patterns and a factor analysis was conducted to determine an underlying structure for all items. As in the first measurement, the item was treated as a single variable and not combined with other like constructs. A principal component analysis was conducted utilizing a varimax rotation. The initial analysis retained six components. Four criteria were used to determine the appropriate number of components to retain: eigenvalue, variance, scree plot, and residuals. This criteria indicated retaining three components. Thus, a principal components analysis was conducted to retain four components and apply the varimax rotation.

After rotation, the three components accounted for $47 \%$ of the explained variance, and all component loadings were positive. The variance explained and reliability score 
(Cronbach's Alpha) by the components is detailed in Table 16. The overall reliability score of the JDS instrument with this data set is .748.

\begin{tabular}{|c|c|c|c|}
\hline Component Loadings & $\frac{\text { Variance }}{\text { Explained }}$ & $\frac{\text { Cronbach's }}{\text { Alpha }}$ & Items loaded description \\
\hline 1 - Job Variety & $23 \%$ & 0.68 & Variety of work tasks \\
\hline 2 -Feedback & $12 \%$ & 0.79 & Feedback \\
\hline 3-Job Autonomy & $12 \%$ & 0.75 & Own decision of work \\
\hline
\end{tabular}

Table 16 - JDS Component loadings - second measurement

The first JDS component consists of job variety items and one feedback from the job item. The job feedback is the extent to which the job provides information about the work performance. The second component reflects how much information of job performance comes from the supervisor, and the third component of autonomy describes how much the individual has the ability to decide how to work.

The original research model proposes a positive relationship between all but the TTF confusion variable with the JDS variables. In the second measurement, two variables have a significant negative relationship with a JDS factor: system compatibility and job identity, and currency with variety. As system compatibility increases, the work in the job is segmented into pieces. The job identity variable is the ability to contribute to the "whole" piece of work, as the system may focus on components of a job rather than the entire work process. The negative relationship between variety and the "real-time" aspect of the data may imply that variety comes from working with aged data rather than current data. The ability and frequency to do problem-solving activities may decrease with more current data, potentially leading to less job variety. 
The correlation and significance level found in the correlation Table (16) confirms the significant positive relationships (.01) for all of the TTF variables with at least one of the JDS variables. System detail significantly correlates with all of the JDS variables.

The strongest positive significant correlation between the TTF and JDS variables is the relationship between system presentation and supervisor feedback. The correlations between the TTF and JDS variables are located in Table 17.

\begin{tabular}{|c|c|c|c|c|c|c|}
\hline \multicolumn{7}{|c|}{ Job Diagnostic Survey (JDS) } \\
\hline & w/Others & Autonomy & Identity & Variety & Significance & Feedback \\
\hline Ease_of Use & 0.12 & 0.01 & -0.02 & 0.15 & 0.06 & 0.14 \\
\hline$p$-value & 0.12 & 0.47 & 0.41 & 0.07 & 0.27 & 0.07 \\
\hline Location & -0.10 & 0.21 & 0.02 & 0.12 & 0.18 & 0.15 \\
\hline$p$-value & 0.15 & 0.02 & 0.44 & 0.13 & 0.04 & 0.06 \\
\hline Compatibility & 0.22 & -0.14 & -0.17 & -0.02 & -0.05 & -0.06 \\
\hline$p$-value & 0.01 & 0.07 & 0.04 & 0.41 & 0.32 & 0.29 \\
\hline Accuracy & 0.17 & 0.16 & -0.09 & 0.06 & 0.04 & 0.08 \\
\hline$p$-value & 0.05 & 0.06 & 0.19 & 0.29 & 0.36 & 0.22 \\
\hline Currency & 0.07 & -0.26 & -0.18 & -0.21 & -0.20 & -0.33 \\
\hline$p$-value & 0.24 & 0.00 & 0.04 & 0.02 & 0.02 & 0.00 \\
\hline Access & -0.02 & 0.21 & 0.08 & 0.14 & 0.17 & 0.28 \\
\hline p-value & 0.42 & 0.02 & 0.21 & 0.08 & 0.05 & 0.00 \\
\hline Confusion & 0.30 & -0.06 & -0.06 & -0.09 & -0.06 & -0.04 \\
\hline p-value & 0.00 & 0.28 & 0.28 & 0.18 & 0.27 & 0.33 \\
\hline Reliability & 0.03 & 0.11 & -0.10 & -0.01 & 0.10 & 0.01 \\
\hline$p$-value & 0.39 & 0.14 & 0.15 & 0.46 & 0.16 & 0.46 \\
\hline Assistance & 0.07 & 0.22 & 0.11 & 0.28 & 0.20 & 0.33 \\
\hline$p$-value & 0.25 & 0.01 & 0.13 & 0.00 & 0.02 & 0.00 \\
\hline Detail & 0.15 & 0.32 & 0.17 & 0.31 & 0.27 & 0.39 \\
\hline$p$-value & 0.07 & 0.00 & 0.05 & 0.00 & 0.00 & 0.00 \\
\hline Meaning & 0.04 & 0.30 & 0.03 & 0.10 & 0.29 & 0.23 \\
\hline$p$-value & 0.34 & 0.00 & 0.40 & 0.15 & 0.00 & 0.01 \\
\hline Presentation & 0.03 & 0.33 & 0.11 & 0.18 & 0.24 & 0.28 \\
\hline$p$-value & 0.39 & 0.00 & 0.13 & 0.04 & 0.01 & 0.00 \\
\hline
\end{tabular}

Table 17 - Job Diagnostic Survey and Technology Task Fit survey Correlations for the second measurement 
The factor loadings were also tested for significant correlations and the results appear in Table 18. The only TTF system factor that was not correlated significantly (at the .10 level) with the JDS factors is compatibility.

\begin{tabular}{|clccc|}
\hline \multicolumn{5}{c|}{ Job Diagnostic Survey (JDS) Factors } \\
Job & Work \\
Variety & Feedback & w/others \\
Technology & LookFeel & 0.18 & 0.30 & 0.17 \\
Task Fit & p-value & 0.05 & 0.00 & 0.05 \\
Survey & Compat & 0.00 & -0.15 & -0.14 \\
Factors & p-value & 0.49 & 0.08 & 0.09 \\
& Detail & 0.29 & 0.29 & 0.22 \\
& p-value & 0.00 & 0.00 & 0.02 \\
\hline
\end{tabular}

\section{Table 18 - Component Correlations TTF and JDS}

In the first measurement, the correlation between the JDS and OCI variables differed from published relationships, and in the second measurement, the same difference occurs. The strongest relationships are seen between the JDS feedback variable and the constructive variables of humanistic and achievement; these correlations are listed in Table 19.

As in the first measurement, the first data reduction returned 30 factors with eigen values above 1.0. In an effort to reduce the number of variables, several factor models were tested. The best raw data model loaded all of the items into five forced factors for a $46.58 \%$ variance explanation. After further analysis, the fifth factor was dropped. The reliability of each component is listed in Table 20 . 


\begin{tabular}{|c|c|c|c|c|c|c|}
\hline \multicolumn{7}{|c|}{ Job Diagnostic Survey (JDS) } \\
\hline & w/Others & Autonomy & Identity & Variety & Significance & Feedback \\
\hline Achieve & 0.017 & 0.306 & 0.11 & 0.166 & 0.31 & 0.29 \\
\hline$p$-value & 0.433 & 0.001 & 0.133 & 0.048 & 0.001 & 0.001 \\
\hline Self act & -0.097 & 0.202 & 0.058 & 0.127 & 0.262 & 0.295 \\
\hline$p$-value & 0.165 & 0.019 & 0.278 & 0.102 & 0.004 & 0.001 \\
\hline Human & -0.07 & 0.233 & 0.138 & 0.266 & 0.215 & 0.332 \\
\hline p-value & 0.242 & 0.008 & 0.082 & 0.003 & 0.015 & 0.000 \\
\hline Affiliate & 0.009 & 0.21 & 0.128 & 0.132 & 0.218 & 0.205 \\
\hline p-value & 0.463 & 0.016 & 0.097 & 0.093 & 0.014 & 0.017 \\
\hline Opposition & 0.111 & -0.161 & -0.166 & 0.008 & -0.162 & -0.203 \\
\hline$p$-value & 0.133 & 0.05 & 0.046 & 0.467 & 0.051 & 0.018 \\
\hline Competitive & 0.106 & -0.113 & 0.007 & 0.049 & 0.025 & -0.074 \\
\hline p-value & 0.143 & 0.125 & 0.471 & 0.311 & 0.402 & 0.226 \\
\hline Power & 0.093 & -0.172 & -0.156 & -0.09 & -0.068 & -0.187 \\
\hline$p$-value & 0.176 & 0.04 & 0.057 & 0.185 & 0.246 & 0.027 \\
\hline Perfect & -0.195 & 0.193 & 0.005 & 0.09 & 0.264 & 0.162 \\
\hline$p$-value & 0.024 & 0.024 & 0.481 & 0.183 & 0.004 & 0.048 \\
\hline Conventional & -0.088 & 0.065 & -0.02 & -0.114 & 0.004 & -0.137 \\
\hline$p$-value & 0.189 & 0.257 & 0.422 & 0.127 & 0.486 & 0.08 \\
\hline Avoidance & -0.04 & -0.076 & -0.075 & -0.112 & -0.191 & -0.243 \\
\hline$p$-value & 0.346 & 0.22 & 0.223 & 0.131 & 0.026 & 0.006 \\
\hline Approval & 0.007 & 0.183 & 0.14 & 0.202 & 0.21 & 0.185 \\
\hline$p$-value & 0.473 & 0.031 & 0.078 & 0.021 & 0.017 & 0.029 \\
\hline Dependent & -0.111 & 0.139 & 0.007 & -0.009 & 0.167 & 0.053 \\
\hline p-value & 0.132 & 0.079 & 0.471 & 0.465 & 0.046 & 0.294 \\
\hline
\end{tabular}

Table 19-Job Diagnostic Survey and Organization Culture

Inventory correlations in the second measurement

\begin{tabular}{|lllll|}
\hline Component Loadings & $\frac{\text { Variance }}{\text { Explained }}$ & & Cronbach's & \\
\cline { 1 - 2 } 1- Constructive & $\frac{\text { Alpha }}{20.02 \%}$ & & & Items loaded description \\
2- Aggressive & $12.84 \%$ & & 0.85 & Collection of all constructive items \\
3- Passive & $5.37 \%$ & 0.85 & Collection of aggressive items \\
4- Avoidance & $5.28 \%$ & 0.78 & Collection of passive items \\
\hline
\end{tabular}

Table 20 - OCI Component loadings - second measurement 
The first component is made up of 39 items from the constructive dimensions of achievement, self-actualizing, humanistic-encouraging, and affiliate. The second component represents the aggressive/defensive style and has 36 items from the competitive dimension. The third and fourth components come from the passive/defensive style. The third component has 31 items from avoidance and the fourth component contains 5 items from avoidance. The correlations of each factor are listed in Table 21 .

\begin{tabular}{|c|ccccc|}
\hline \multirow{3}{*}{ Job } & \multicolumn{5}{|c|}{ Technology Task Fit Survey Factors } \\
& & LookFeel & Compat & Detail & SysChar \\
Diagnostic & Job Variety & 0.18 & 0.00 & 0.29 & -0.04 \\
Survey & $p$-value & 0.05 & 0.49 & 0.00 & 0.36 \\
(JDS) & Feedback & 0.30 & -0.15 & 0.29 & 0.00 \\
Factors & $p$-value & 0.00 & 0.08 & 0.00 & 0.49 \\
& Work with others & 0.18 & -0.14 & 0.22 & 0.14 \\
& $p$-value & 0.05 & 0.09 & 0.02 & 0.09 \\
\hline & Constructive & 0.31 & -0.05 & 0.40 & 0.01 \\
Organization & $p$-value & 0.00 & 0.35 & 0.00 & 0.48 \\
Culture & Aggressive & -0.10 & 0.25 & 0.02 & 0.20 \\
Inventory & $p$-value & 0.21 & 0.01 & 0.43 & 0.04 \\
Factors & Passive & 0.04 & 0.06 & 0.14 & 0.10 \\
& $p$-value & 0.36 & 0.32 & 0.11 & 0.19 \\
\hline
\end{tabular}

Table 21 - Correlations of all factors -second measurement

Path Analysis

After the second measure, path analysis regressions were conducted to determine the causal effects among the system characteristics, job factors, and OCI factors. Ten paths were significant $(p<.10$ level). Three of them were between the TTF factor of look and feel of the system and all of the JDS factors, variety (beta $=.178, p=.09$ ), feedback (beta $=.304, p=.00)$, and work with others (beta $=.171, p=.01$ ). Three paths were between system detail and the JDS factors of variety (beta $=.323, p=.01$ ), feedback 
(beta $=.308, p=.09$ ), and work with others (beta $=.229, p=.03$ ). The only significant path for the JDS factor variety was with the OCI factor constructive (beta $=.275, p=$ $.01)$. The JDS feedback factor had two paths - one path was positive leading to the $\mathrm{OCI}$ aggressive factor (beta $=.564, p=.00$ ) and the second was negative leading to the OCI factor of passive (beta $=-.196, p=.07$ ). The JDS work with others had one path to the OCI factor of passive (beta $=.234, p=.04$ ). The path coefficients and p-values can be seen in Figure 6.

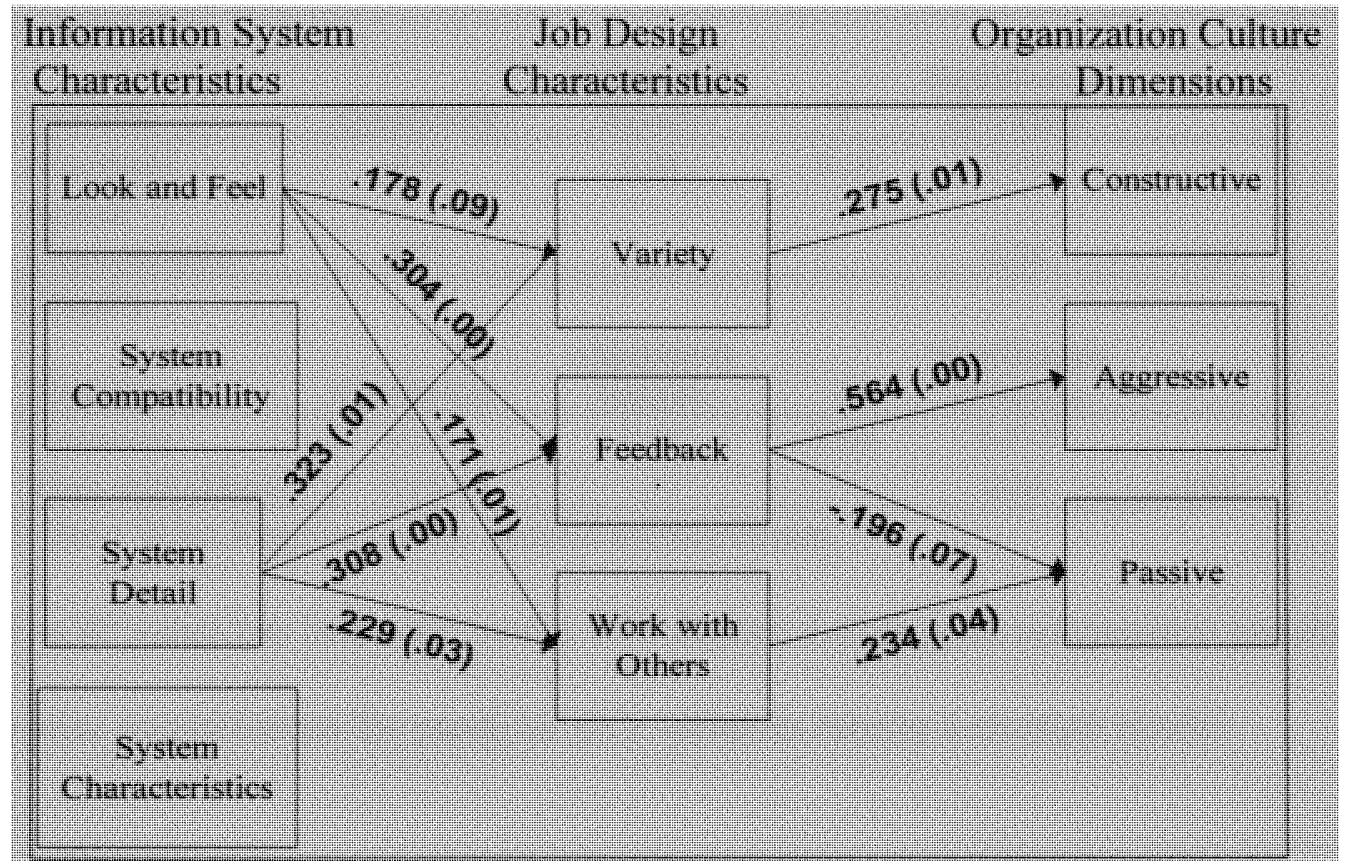

Figure 6 -Second measurement path analysis

To further understand the correlation among several variables, regression analysis is used to eliminate conjoint correlations and eliminate significant, but trivial, correlations. Direct regression was also tested between the TTF and JDS factors and the OCI factors. A step-wise regression method was used to return only the factors that have a significant relationship with the dependent variable. Only three of the OCI factors had 
significant relationships with combinations of the TTF factors and JDS factors. Table 22 reports the results of the regression analysis.

\begin{tabular}{|c|c|c|c|c|c|c|}
\hline \multicolumn{7}{|c|}{ Factor 1: Constructive } \\
\hline Model & Variable & Coefficient & $\begin{array}{l}\text { Adjusted R } \\
\text { Square }\end{array}$ & $\mathbf{F}$ & $\begin{array}{c}\text { Significance } \\
(p \text {-value })\end{array}$ & VIF \\
\hline \multirow[t]{2}{*}{1} & (Constant) & -0.03 & 0.34 & 37.25 & 0.759 & \\
\hline & Feedback & 0.57 & & & 0.000 & 1.00 \\
\hline \multirow[t]{3}{*}{2} & (Constant) & -0.03 & 0.38 & 23.04 & 0.789 & \\
\hline & Feedback & 0.50 & & & 0.000 & 1.11 \\
\hline & Detail & 0.27 & & & 0.016 & 1.11 \\
\hline \multicolumn{7}{|c|}{ Factor 2: Aggressive } \\
\hline Model & Variable & Coefficient & $\begin{array}{l}\text { Adjusted R } \\
\text { Square }\end{array}$ & $\mathbf{F}$ & $\begin{array}{c}\text { Significance } \\
(p \text {-value })\end{array}$ & \\
\hline \multirow[t]{2}{*}{1} & (Constant) & -0.04 & 0.04 & 4.10 & 0.72 & \\
\hline & $\begin{array}{l}\text { System } \\
\text { Characteristics }\end{array}$ & 0.23 & & & 0.04 & 1.00 \\
\hline \multicolumn{7}{|c|}{ Factor 3: Passive } \\
\hline Model & Variable & Coefficient & $\begin{array}{c}\text { Adjusted R } \\
\text { Square }\end{array}$ & $\mathbf{F}$ & $\begin{array}{c}\text { Significance } \\
(p \text {-value })\end{array}$ & \\
\hline \multirow[t]{2}{*}{1} & (Constant) & 0.05 & 0.06 & 5.26 & 0.717 & \\
\hline & Work with Others & 0.28 & & & 0.03 & 1.00 \\
\hline
\end{tabular}

Table 22- Step Wise Regression for second measurement

The $\mathrm{R}^{2}$ value has increased on the TTF factors. Loading all independent variables in a step-wise regression on the predictor OCI variables returns a stronger model.

\section{Difference Dataset}

A dataset consisting of the measurement of the difference between the first and second measurement (first response - second response) was also used in the analysis. After computing several of the multivariate tests, it's use was discontinued due to the similarities found with the second measurement dataset. The dataset provided no additional information on the results. 
In order to evaluate the significance of the changes in the environment, a matched pair analysis was conducted on the ninety-six individuals who completed the survey during both measurements. An F-test analysis was conducted to determine if the group covariances are equal and the total group means are different between the first and second measurement. The test revealed that the two group means of all variables were not equal $(F=2.721, p<.008)$. Each summed variable was tested and then transformed for normality. This was necessary to conduct the T-test. If the variable was too extreme and not able to be normalized, a Wilcox non-parametric analysis was conducted. The results of the analysis are located in Table 23.

Four categories of responses to the null hypothesis are presented. Mean values with an absolute significantly difference between the legacy and ERP measurement periods are indicated with a "Difference" label and no mean differences are labeled "No Difference". Means that are positively significant only are labeled "+ Difference". Likewise, means that are negatively significant only are labeled "- Difference". Bold indicates an alpha value of .10 is used to judge significance. Italics indicate a liberally applied alpha value of .15 , creating a second group of statically significant variables. Six TTF variable differences were found to be significant when comparing the two measurement periods. Ease of use, help, meaning, and presentation were found to be lower in the ERP environment. This corresponds with notion of a new system being unknown. The lower means are of the ERP are expected given the high TTF variable 
means of the legacy system. Only the variables of system compatibility, system accuracy, system currency, and system reliability failed to be different.

\begin{tabular}{|c|c|c|c|c|c|c|}
\hline & & Matched & airs Tabl & $=96$ & & \\
\hline & & & & t-test & & \\
\hline Legacy (a) & Overall & & & Diff ! = & & $\mathbf{H}^{0}:$ No \\
\hline $\operatorname{ERP}(b)$ & Change & Wilcoxon & Diff $<0$ & 0 & Diff $>0$ & Difference \\
\hline TTF Variables & & & & & & \\
\hline Ease of Use $(a-b)$ & LOWER & 0.005 & 0.957 & 0.085 & 0.042 & Difference \\
\hline Location $(a-b)$ & LOWER & 0.280 & 0.854 & 0.292 & 0.146 & + Difference \\
\hline Compatibility $(a-b)$ & HIGHER & 0.640 & 0.492 & 0.980 & 0.508 & No Difference \\
\hline Accuracy $(a-b)$ & LOWER & 0.882 & 0.572 & 0.856 & 0.428 & No Difference \\
\hline Currency $(a-b)$ & HIGHER & 0.604 & 0.448 & 0.896 & 0.552 & No Difference \\
\hline Access $(a-b)$ & LOWER & 0.164 & 0.890 & 0.218 & 0.109 & + Difference \\
\hline Confusion $(a-b)$ & HIGHER & 0.008 & 0.007 & 0.014 & 0.993 & Difference \\
\hline Reliable $(a-b)$ & LOWER & 0.404 & 0.819 & 0.362 & 0.181 & No Difference \\
\hline Help $(a-b)$ & LOWER & 0.000 & 0.999 & 0.000 & 0.000 & Difference \\
\hline Detail $(a-b)$ & HIGHER & 0.126 & 0.930 & 0.139 & 0.069 & + Difference \\
\hline Meaning $(a-b)$ & LOWER & 0.004 & 0.001 & 0.003 & 0.998 & Difference \\
\hline Presentation $(a-b)$ & LOWER & 0.006 & 0.997 & 0.006 & 0.003 & Difference \\
\hline JDS Variable & & & & & & \\
\hline Deal w/Others $(a-b)$ & HIGHER & 0.570 & 0.523 & 0.950 & 0.476 & No Difference \\
\hline Autonomy $(a-b)$ & LOWER & 0.830 & 0.869 & 0.260 & 0.130 & + Difference \\
\hline Identity $(a-b)$ & LOWER & 0.340 & 0.870 & 0.250 & 0.129 & + Difference \\
\hline Variety $(a-b)$ & HIGHER & 0.000 & 0.020 & 0.040 & 0.980 & Difference \\
\hline Significance $(a-b)$ & LOWER & 0.000 & 1.000 & 0.000 & 0.000 & Difference \\
\hline Feedback $(a-b)$ & LOWER & 0.240 & 0.887 & 0.220 & 0.112 & + Difference \\
\hline OCI Dimensions & & & & & & \\
\hline Oppositional $(a-b)$ & LOWER & 0.864 & 0.616 & 0.767 & 0.383 & No Difference \\
\hline Power $(a-b)$ & LOWER & 0.338 & 0.912 & 0.178 & 0.087 & + Difference \\
\hline Competitive $(a-b)$ & LOWER & 0.529 & 0.735 & 0.545 & 0.264 & No Difference \\
\hline Perfect $(a-b)$ & HIGHER & 0.213 & 0.996 & 0.673 & 0.663 & No Difference \\
\hline Achievement $(a-b)$ & HIGHER & 0.850 & 0.258 & 0.516 & 0.741 & No Difference \\
\hline SelfAct $(a-b)$ & HIGHER & 0.918 & 0.448 & 0.861 & 0.559 & No Difference \\
\hline Humanistic $(a-b)$ & HIGHER & 0.890 & 0.204 & 0.409 & 0.795 & No Difference \\
\hline Affliative $(a-b)$ & HIGHER & 0.702 & 0.115 & 0.231 & 0.884 & - Difference \\
\hline Approval $(a-b)$ & LOWER & 0.333 & 0.706 & 0.586 & 0.545 & No Difference \\
\hline Convent $(a-b)$ & LOWER & 0.005 & 0.993 & 0.013 & 0.006 & Difference \\
\hline Dependent $(a-b)$ & LOWER & 0.002 & 0.999 & 0.001 & 0.000 & Difference \\
\hline Avoid $(a-b)$ & LOWER & 0.670 & 0.668 & 0.663 & 0.331 & No Difference \\
\hline
\end{tabular}

Table 23 - Matched Pair Analysis Results 
The TTF system confusion and data detail variables produced a higher mean than in the legacy environment. The new ERP system provides a current data-rich environment that is not found in the old environment. It is one of the major differences between the two technologies. The higher confusion variable confirms the lack of individual and organizational knowledge yet to be created to support the new ERP environment.

In the JDS variables, two variables were significantly different. The variety variable has a significantly higher mean in the second environment. This may be the result of work process change due to the new information system or as the way information about the new system was being dispersed. The significant variable tested significantly lower than the first measurement. Three variables are significant if the testing alpha level is increased to .15. Autonomy, identity, and feedback each show a significantly lower mean value in the ERP. The ERP environment did produce a different set of job design characteristics perceptions; therefore, we reject the null hypothesis that there is no change found between the legacy and ERP environments.

A mixed result is found when examining the differences between the organizational culture dimensions in the legacy and ERP environment. One aggressive/defensive norm, power, and two passive/defensive norms, conventional and dependence, tested significantly lower in the ERP environment. Three constructive dimensions: self-actualization, humanistic, and affliative tested showed an increase in value in the ERP environment, however, they did not test significantly different from the legacy environment. Once again applying a liberal testing alpha level of .15, the affliative variable shows a significantly higher mean in the ERP environment. Out of the twelve 
dimensions, three dimensions tested significantly different in the ERP environment. There was a significant change in one third of the culture dimensions. The hypothesis suggesting change in the organizational culture is only partially supported.

Data Analysis Summary

There is support for the two sets of questions with the results of the first and second measurements. The first question of understanding how the perceived system characteristics align with an individual's perceived job design characteristics is supported by each of the system characteristics significantly correlated in the proposed direction. The stronger support of this relationship becomes visible in the causal flow of the effects seen in the path diagram. System characteristics do contribute to job perception. The second question of the perceived job design characteristics aligning with dimensions of organizational culture is not as strong as the literature suggests. Although there are significant correlations between the JDS and OCI variables, only one relationship can be seen in the causal flow. The matched pair analysis supports the third question of differences between the two environments. There is a significant difference in perceived job design characteristics between the legacy information system environment and the new ERP environment. There is partial support for a difference in organizational culture between the old and new information system environment.

In Chapter 3, 89 hypotheses were proposed. Each hypothesis described a direct association for each of the independent variables with the TTF and JDS constructs and the dependent variable of organizational culture. The statistical analyses of both measurement periods indicate support for a direct relationship of TTF variables with JDS 
variables. Connections between TTF variables and the OCI variables also reveal relationships. (The specific hypotheses are repeated in the summary of hypothesis table with an indication as to whether they were supported or not supported in the study located in the appendix.)

Furthermore, differences in perceptions of technology, job characteristics, and organizational culture dimensions as defined by the OCI instrument are supported by the matched pair analysis. Although only a third of the dimensions are supported for change, three of the four constructive dimensions showed a positive, but not significant, increase. This difference is important. The constructive dimensions in the legacy environment were the lowest of the three norms. Having the majority of the norm show a movement to increase implies that the new ERP environment is supporting and encouraging this type of behavior. This is a significant change from the prior environment. A different set of behaviors is being supported in the new ERP environment.

The path analysis supports a causal relationship of the variables in both the first and second measurement. The direct relationship between all variables does not return a good model of explanation for the observed variance. The highest $\mathrm{R}^{2}$ obtained was in the second measurement in predicting the constructive culture by the variables system detail and job feedback.

The low $\mathrm{R}^{2}$ values found in the regression models may be indicators of other latent or unmeasured variables existing in the environment and impacting the outcome of the predicted $\mathrm{Y}$ value of organizational culture. Or the values could be weaknesses of the individual instrument weaknesses in capturing the observed variable. The TTF instrument has an established history of low $\mathrm{R}^{2}$ values; the predicted values for the instrument range 
from .10 to .25 . This inability to strongly associate technological concepts with how individuals perceive their jobs and organizational culture would lower the overall predictive values of the models. The lower values of correlations between the JDS and $\mathrm{OCI}$ styles is also a predictive outcome based on previous studies that predict low ranges (from -.29 to .37).

On the balance, the results of the numerous statistical tests provide support for many of the hypothesized relationships in the research model, thus confirming that information systems affect job design characteristics, and directly and indirectly, organization culture dimensions. A discussion of these findings follows in the next chapter. 
VI. Discussion

The objective of this chapter is to present a discussion of the findings, limitations, and future directions of this research. The discussion section is organized into two sections: perceptions of each environment and perceived differences between the legacy and ERP environment. This is followed by limitations and future directions.

Legacy Environment

The first measurement data was collected to understand the perceptions of the legacy system and the working legacy environment before the technology change. The users were asked to reflect on how they used the legacy system in their job, how they felt about different dimensions of their job, and how they saw co-workers behaving in the working environment.

The total group of respondents evaluated the legacy systems as providing a moderate level of fit to the individual's job tasks (mean $=4.58$ in a range of 1 to 7 ). The moderate task fit of the current system is supported by several key usability variable ratings of system reliability, presentation, and ease of use. Given that the system had been used for more than 15 years, the users had grown accustomed to navigation and the overall appearance of the system. The highest degree of fit was found in the variable describing the ease of obtaining system assistance in the environment (assistance mean= 5.07 out of the maximum score of 7). This degree of fit in system assistance indicates a high level of user knowledge about the system and how it supports tasks in the department. This measure indicates that the users were confident in their workings with the system. This confidence is also reflected in the first factor of system characteristics. 
The factor analysis of the information characteristics indicated the strongest factor was a collection of system traits including the level of system data detail to support the work task, the location of data in the system, the ease of use, and the implied meaning of the system data and supporting functionality. The collapsing of the set of variables into one factor implies a common perception for those system characteristics that best support the work task. The system had been customized over a long period of time to fit the required tasks of the organization. This is a normal occurrence for information systems that have been in the work environment for an extended period of time and are able to be modified by workers. Users would have grown accustomed to the necessary steps using the information system to support the work task. A comment from one of the participants reflects the system characteristics factoring result found.

"Legacy, which was the old system, was easier, you had a little specifying and then bam, you were there. Even though you switched the screens, they were accessible. You didn't even have to click on a mouse. You just, you know, did something on the keyboard and you were there."13

The second and third information system characteristic factors related to the comparison of the information contained in the legacy system with other data sources and evaluating the accuracy. The second factor contained items that confirmed the legacy system data created confusion when being checked against other sources, and the third factor evaluated the accuracy of the legacy system data. Given that the system was a collection of non-integrated databases that stored duplicate information, user confusion would have been a common occurrence in the working environment. This explanation supports the low mean value of system currency (mean $=3.40$ with maximum score of 7 ).

\footnotetext{
${ }^{13}$ From the interview with participant F1001, 7/22/04.
} 
System currency items measured the system's ability to provide current data to support tasks. The legacy system was updated on an overnight or bi-overnight schedule and could not immediately reflect changes in the data. With multiple databases and untimely updates, the data would be in a constant state of flux. It would be necessary to adjust the work process to allow for this flux creating additional job variety and, when uncertain of timing, confusion about what information was correct.

Although the system created confusion, it was reliable and able to perform as necessary as seen in the last two factors of system reliability and system performance. The factor measuring reliability of the system is significantly positively correlated with the JDS factor of variety. This reflects how system work impacted the diversity of the operating environment. When the system was not functional, the variety of the job task would decrease. This same factor is negatively correlated with the competitiveness and avoidance factors. Having the system operational impedes on the ability to avoid work tasks and allows access to information. Competitive behavior is supported by limiting access or having no access to information that is needed for the work task. The information system characteristic factors accurately describe the legacy system and how it worked in the environment.

The total legacy respondent group evaluated each of the JDS within the mid-range of the survey scale. The small consistent range of responses is reflected in the low standard deviations measure. The factor analysis reflected similar strengths of the JDS variables factoring feedback, variety, autonomy, and significance into separate groups. Unlike the information system characteristics factors, the JDS factors loaded each of the corresponding items into the proper factor. There was no cross-over of items. The 
strongest factor in the legacy environment is feedback. Job performance is understood through indicators found in the tasks such as feedback from the system in the form of reporting or physically checking information with others. The lack of the interaction variable of "work with others" implies that coordination actions are not actively sought or perhaps encouraged. This is also echoed in the third factor of autonomy were it seems important in this environment to be self-sufficient. The second factor of variety and fourth factor of significance demonstrates how different job tasks that are regarded as important to the environment are a reflection of the job. In this environment, workers like to act on their own, have varied but important responsibilities, and look for feedback from the task.

The OCl instrument provides a measurement of how members believe they should interact with one another carrying out their work and meeting the expectations of their supervisors. The summary of the impressions is best interpreted in three categories that group the twelve dimensions. The categories are constructive, passive/defensive, and aggressive/defensive cultures. Each organization has some degree of each type of culture. The strength of the grouped category is compared to the national norms to determine what types of behaviors are perceived as common behaviors.

The passive/defensive styles are the strongest set for the total group with the conventional and dependent dimensions ranking the highest $\left(75^{\text {th }}\right.$ percentile). The remaining group dimensions also rank high at $74^{\text {th }}$ for avoidance and $62^{\text {nd }}$ for approval. In this culture, members believe they must interact with others in defensive ways that will not threaten their own security. Each of the sub-groups reflects similar measurements and ranking in this culture category. The strength of the measurement suggests that current 
operating environment elements (such as job characteristics and system usage) support these types of behaviors. Support for passive/defensive dominance comes through in the interviews in the form of comments on management behavior. Several participants commented on passive, myopic behaviors:

"There is a lot of lack of communication. I mean, what goes on, on the other side of the building, they don't communicate with us, again, we find out and we hear whenever we talk to the evaluators. "Oh yeah, we're not doing this anymore." I'm not saying it happens all the time, but I mean, the few times that it happens, it's very important that we should know these things, that I feel that "What is happening, why can't they just email us, send us a memo, so we can sign on it."14

"...when it comes to the rest of the office as all 30 people working together, again, I find that to be a little bit conflictive. It becomes a problem because, you know, someone commandeers for certain things, and everyone's trying to find where, along the process, things have gone wrong. And it takes a while to go through every single department...But when it comes to the whole office, when it gets bigger, because it's kind of that age-old thing, the bigger the group is, the harder it is to control, and that really follows, in an organization it happens the same way." 15

The factor analysis of the $\mathrm{OCI}$ instrument identified the constructive construct as the variable explaining the majority of the item variance. The majority of the items in the constructive styles grouped into this one factor unlike the other factors that only contained single dimension items. The $\mathrm{OCI}$ instrument measures the summed totals of the dimensions; the higher the sum of the dimension, the stronger a behavior is seen in the operating environment. The factor analysis analyzes all sources of variability - unique, shared, and error - for each observed item. Constructive behaviors are seen in the environment with more diversity and with no sustaining support than the other two styles.

\footnotetext{
${ }^{14}$ From the interview with participant A1010, 7/28/04.

${ }^{15}$ From the interview with participant $\mathrm{A} 1010,7 / 28 / 04$.
} 
The dominant style of passive/aggressive is the first set of behaviors that are re-enforced and commonly accepted in the environment.

The first measurement path analysis determines several important conclusions regarding the relationships between information system environment factors, job characteristics factors, and organizational operating culture dimension factors. The first is the system characteristics factor supports the perception of feedback, autonomy, and significance of the job in the operating environment. A positive relationship is found between how the system supports the work task through the system ease of use, system location or accessibility, data meaning, and data detail. System characteristics directly support feedback and indirectly support the OCI constructive factor. In addition to the feedback factor, system characteristics also support the perception of significance, and to a lesser extent, of job autonomy. This implies that a degree of meaning is placed with working with the system within the operating environment. Jobs with the need for higher system interaction or increased data access may be perceived to be of greater importance in the organizational structure. The second is that a negative relationship was found between the system accuracy factor and the job characteristic factor of feedback. Poor or inaccurate system information detracts or reduces feedback from the job. The ability to understand one's performance is decreased when the system data is inaccurate or misleading. Actions to support the bad data may lead to unproductive action and further problems in the work task. The negative relationship also detracts from the job characteristic factor of feedback by indirectly reducing the overall level of support of this factor and the constructive behaviors factor found in the operating environment. The final conclusion can be drawn from the lack of supporting relationships seen between the 
factors. Only two of the five information system environment characteristic factors have significant correlations with factors of the job characteristics. This implies that the factors do not play a large role in reinforcing job characteristics in the legacy environment. The lacking connection between any of the information system characteristics factor and the JDS factor of variety confirms that the system was well known in the support of necessary tasks.

The legacy system was considered a positive but insubstantial influence on the work environment. It contributed to the perception of positive job characteristics and constructive operating behaviors. The feelings of the old system are summed up nicely in the comments from one of the research participants:

“...I've always... been saying, "Look, we had Legacy." Yeah, it had some major deficiencies and things, but one thing that it was perfect at was being simple and like, concise. As an application, you got on one screen and you could see everything, from citizenship to date of birth to their address to, you know, and then with just the flick of two fingers, and two letters, click between four different screens and see different data. Let me see what grades you got now, and see [the] last grade, let me see if you're immunized, and you're done." 16

Overall, the legacy environment seems to be a stable state of known passive/aggressive behaviors. Having one JDS item demonstrate a significant correlation with the OCI factors shows employees seeing their jobs containing the elements of variety and autonomy but not seeing the connections between their job and the greater organization behavior. Constructive behaviors seemed to be a novelty and not well supported.

There is support for the two main questions in the legacy environment. The first question of understanding how the perceived system characteristics align with an individual's perceived job design characteristics is supported by each of the system

\footnotetext{
${ }^{16}$ From the interview with participant R1001, 7/27/04.
} 
characteristics significantly correlated in the proposed direction. The stronger support of this relationship becomes visible in the causal flow of the effects seen in the path diagram and in the step-wise regression. System characteristics do contribute to job perception. The second question of the perceived job design characteristics aligning with dimensions of organizational culture is not as strong as the literature suggests. Although there are significant correlations between the JDS and OCI variables, only one relationship can be seen in the causal flow. The third question will be addressed in the differences section later in this chapter. In the next section, each of these constructs will be re-examined after introduction and usage of the new system.

\section{ERP Environment}

The second measurement data was collected after the implementation of the new ERP system, where the system was being used by all survey participants in the working environment. The users were asked to reflect on how they were using the new system in their job, how they felt about different dimensions of their job, and how they saw coworkers behaving in the working environment. As in the first section, the interviews are used to assist in the validation of the empirical findings.

The evaluation of the new system implies a moderate level of fit to the individual's job tasks. (The TTF variables mean's range from 3.5-4.7 on a scale of 1-7.) This is surprising considering the system was still undergoing many changes and the users had not had much experience using the new system. The moderate task fit of the current system is supported by several key usability variable ratings of system compatibility, ease of use, system data detail, and presentation. The highest degree of fit 
was found in the variable describing the compatibility of the system with their work task (compatibility mean $=4.72$ ).

The factor analysis of the information system characteristics in the second measurement indicated that the strongest factor was how the system presented information. The items in the factor include system presentation, system ease of use, and system meaning. The collapsing of the set of variables into one factor implies a common perception for those system characteristics that support the presentation of information and data. There is a striking difference between the old and new system display and it is well represented by the strength of this factor. The second strongest factor was the concept of compatibility of the system in their job. The factor contained items confirming that the system supports their work in a form that they understand and are able to use. The third factor contained all of the items of data detail. These items ask if the information is presented by the system in a level of detail to support their work task. The arrangement of data is one of the big differences between the old and new systems. The new system provides data that can be investigated for linkages of data relationship. This "digging" ability provides greater meaning and detail to support work tasks.

The second JDS variable evaluation has ranges between 3.5 and 5.5 for a midrange mean in the survey scale. The factoring of the JDS revealed a different set of significant factors from the first measurement. Job variety factored in the most significant items and explained $23 \%$ of the total variance in the second measurement. The second factor loaded items regarding feedback from the job and two items from the supervisor. This is a different perception of feedback than found in the legacy environment. This importance given to feedback may be a way for employees to gauge if they are using the 
new system correctly. Familiar affirmations for a good performance have changed with the new system. The workers are looking for positive encouragement in many different places. From one participant:

"Well, I think it...is, because like we're implementing a new software. And I think that that's kind of put everybody on edge and has kind of scared people who've been here a long time. And so it's making them come out of their shells and like ask other sections, 'How do you do this?' 'I can't find that.' You know, it's interesting to see that happen and you have other people with it even in our office who typically don't always interact, kind of going, 'Hey, you know, remember we went to that class, and I don't know the PantherSoft,' and I found myself going through a couple of people I normally don't interact, even though we're in the same office, and I'm going, 'Hey, remember when we sat in that. I don't remember how we did this.' And it's not that it's really difficult, it's just that it's new. And so I think it's actually had an interesting effect on changing the environment and the climate, because departments are also responsible for the PantherSoft thing, and what they have to do.",

The impact of the need for increased feedback from the environment also influences the third factor of "working together" which was not represented in the first measurement JDS factoring. This factor is made up of items describing how the employees interact with others during work tasks. The need for information to accomplish the work task in the new system environment has influenced how people interact with one another for assistance and support and is reflected in the OCI factors. The matched pair analysis also supports this new ERP environment behavior with the significant finding of a higher mean of the "work with others" JDS variable. The perception of job design characteristics in the legacy environment is identified as significantly higher in autonomy, identity, variety, significance, and feedback. In the new environment, individuals perceive a reduction of these job characteristics.

\footnotetext{
${ }^{17}$ From the interview with participant R1001, 7/27/04.
} 
The OCI top three factored grouping of items represents a mixture of each of the three styles: constructive, aggressive/defensive, and passive/defensive. The same as in the first measurement, the strongest factor consists of items from the constructive style. This corroborates the JDS factors reflecting the increase in supporting behaviors. The new system may create a need in the environment that supporting behaviors assist in fulfilling. The remaining factors are composed of a collection of items from the aggressive/defensive and passive/defensive rather than the majority of the single items. This indicates a more diverse range of behavior is being seen and supported in the environment rather than just one type of behavior such as avoidance.

The path analysis demonstrates more activity in the second measurement. System characteristics support all of the JDS factors: job variety, feedback, and working with others. Constructive behavior is supported by the JDS factors of variety and feedback. The only negative relationship is between the JDS variable and the passive OCI factor. This is a logical relationship supporting feedback as a negative or suppressing ingredient to the passive style. The more job and supervisor feedback present in the environment, the less avoidance behaviors.

\section{Behavior Changes}

The OCI maps the summed dimensions means to a percentile chart that is composed of the combined scores of earlier participants. The overall dominant style of the University was passive/defensive in both the first and second time period. This indicates that members believe they must interact with others in defensive ways that will not threaten their own security. The second style, and almost equally as strong, was 
aggressive/defensive. Members are expected to approach their work in forceful ways to protect their status and position. The interesting change that can be seen in the second measurement is how the perception of certain dominant behaviors has decreased and constructive behaviors have increased. In the aggressive/defensive style, the variable that tested significantly lower in the matched-pair analysis was the power dimension. Two dimensions in the passive/defensive style, conventional and dependent, are found to have significantly different and lower means. In the constructive style, the three variables of self-actualization, humanistic, and affiliate prove to be significantly higher in the second measurement. This indicates a statically supported change in the perception of these behaviors. One participate comments on the perceived changes:

"The communication definitely has increased. I would have to say that a lot of the employees that I deal with from different departments now are more receptive, more open and friendly to different ideas, or actually just to listen to what you have to say concerning a problem. And I think, in its own funny way, I think PantherSoft has done that because it's kind of shattered everybody's original like processes and functions and whatnot, so everyone has to kind of learn something new. So now they're more receptive to learning what's going on and maybe theirs is a new technique that I don't know about and I actually like it. I like the way the change has kind of broken everybody out of their rut...before, you could go to (omitted) and come in with a certain problem, and they would just tell you 'No,' automatically, because 'I've been here twenty years, I've dealt with everything, I know.' So, I've enjoyed it, especially for me. I mean I've gotten to experience a lot, learn a lot, and I think I've given the same back to other people in the different departments." 18

The factoring analysis reveals the variability of the responses on the humanistic or constructive styles to be the most active in the survey. This style encourages members to interact with others and approach tasks in ways that will help them meet their higherorder needs. Thus, while the dominant behavior remained in the environment, the activity

\footnotetext{
${ }^{18}$ From interview with participant $\mathrm{R} 1111,7 / 27 / 04$
} 
was around constructive behaviors. The introduction of the new system may have triggered the new behaviors to take effect or may have created a disruption to allow additional behaviors.

People using the new system perceive it to offer a reasonable fit to their work task and look at it as a positive addition and change to the environment. In the matched pair analysis, the TTF variables of ease of use, help, meaning, and presentation are all significantly lower in the second measure. This would imply that the new system does not support the work tasks as well as the legacy system. The adjustment period is short given the longevity of the old system and the time that the users had to adjust their activities to support the new system when the survey was conducted. Prior social construction research (Lind and Zmud, 1991; Orlikowski and Gash, 1994) of the linkage between technology influence and user perception shows that individual sense-making leads the way to mutual social sense-making to comprehend the technology within a given cultural context. This research shows that mutual social sense-making is seen in the activity levels of the users in the constructive behaviors and feedback mechanisms. However, the organizational sense-making is not seen as a mutual coordinated effort but rather as an individual activity taking place after the user realizes that there is a need to know. One participant comments on this behavior:

"The new PantherSoft system, its reporting function is structured very differently, and it seems to still have problems. So I'm having a delay as far as getting that information out to the faculty...I've been trying to notice change, you know, way in advance what happens, and I'm starting to figure out that it may still be tasked on me for the next upcoming semesters, and if I don't start learning it, then I'm going to be really behind."19

\footnotetext{
${ }^{19}$ From interview with participant $\mathrm{R} 1111,7 / 27 / 04$
} 
The dominance of the overall cultural behavior patterns may be the reason for the high individual activities to understand the system. If the environment had had more constructive behaviors characteristics, higher group sense-making activities might have been found.

The matched pair analysis also reveals a drop in the mean of four out of seven JDS variables. The perception of their jobs in the new system environment has reduced individual job characteristics. This could be a direct effect of the ERP system characteristics. As discussed in case study review section, the hierarchical nature and complexity of the system tends to inflict control upon the organization in the form of system rules and procedures. This would account for the lower mean of job autonomy. A lower job identity and significance rating may be a reflection of the system's ability to restrain access by work role. The one JDS variable that increased in the new environment was the variety found in the job. This also may be a direct result of the new system's integration feature. As found in the Viskase case study (Rajaagopal, 2002), the one database concept is allowing the entire organization - rather than just an individual department - to share and control information.

\section{Differences}

The third question of in each set of questions compares the relationships of system characteristics, job design characteristics, and organization culture dimensions between both time periods. Several differences are found. First, there is a difference in how the information system characteristic support individual and group work activities. The first information system characteristics factors are important in both environments in 
that they have a positive relationship with several job design characteristics factors. In the legacy environment, the first information system characteristic factor is strongly linked to job feedback and job significance. The perception of performance and contribution is positively influenced by the system characteristics. After the ERP implementation, the first factor of the systems look and feel influences not only individual performance feedback, but also influences the perception of how one works with others in supporting job tasks. This influence of group support is a new dynamic that was not seen in the legacy environment. This support of group work in the ERP environment extends to one other information system factor, system detail. These new information system factors support a set of behaviors that were not supported by the legacy system.

Second, information system characteristics are viewed as a supporting element in the ERP environment. The information system characteristics and three factors in the ERP environment have a positive relationship with the job design characteristics of variety, feedback and working with others. This relationship of more than one information system characteristics positively supporting a job design characteristic does not exist in the legacy environment. The system is perceived to support, and thus influence, the job design characteristics multiple ways. This supports the notion that the information system characteristics contribute to perception of job design characteristics.

The last major difference in the two environments is the amount of activity between the sets of factors. In the legacy setting, only two information system characteristics and one job design characteristic show any interaction with other variables. The lack of interlinking structural elements describes an environment that is not supported by the system and jobs that do not link the greater organization culture 
elements. In the ERP environment, a higher activity of relationships can be seen. The ERP system has introduced new elements into the working environment that has changed the perception of how the job design characteristics and organization culture dimensions are viewed by the workers. This perception of a changed setting is the impact of the system and the change in the tasks to support the work.

The first question proposed by this research was designed to uncover how perceived system characteristics align with the perception of job characteristics. In both the first and second measurement, each of the TTF variables is correlated with one or more of the JDS variables. The only two TTF variables that are negatively correlated with the JDS variables are system data compatibility and system confusion. These are logical relationships with the variables. The system data compatibility refers to how inconsistent the data is across multiple sources. A negative correlation means that the inconsistent data does not support job characteristics. The same is true for the confusion variable. The higher the level of system confusion found in the environment, the less the perception of job characteristics is an expected relationship. The path analysis demonstrates a direct linkage between the system characteristics and JDS factors in this environment.

The second question was based on previous research by Cooke and Szumal (2000) and asks if the job characteristics can be linked to organizational culture dimensions as measured by the $\mathrm{OCI}$ instrument. Although the predicted values of the correlations are not as strong as in previously reported studies, the correlation between many JDS variables and OCI variables is found. The lack of significant linkages between the JDS and OCI factors in the path analysis may indicate the dominance of the 
passive/defense behaviors. The path analysis reveals linkages of variability. There may be a low level of variability found in the dominating behaviors. This is supported by higher standard deviations found in the constructive factors. The new system characteristics show a higher number of significant relationships in the second measurement. The new system supports constructive dimensions more than the passive/defensive or aggressive/defensive dimensions.

The third question addresses the differences in the settings, and three major items are found. Each of these supports the conclusion that the ERP system has introduced new environmental dynamics and influenced the perception of the worker.

This research presents empirical support that the structural element of information systems contributes to an individual's perception of his or her job and his or her perception of the organizational culture. This is the first known study to empirically link these structural elements. Beyond the establishment of linkages, the study also reveals several other organizational responses to the system change. The system change:

- Inspired the majority of the discussion participants to anticipate a change in how the organization views their job and how they work together,

- Was seen as an invisible trigger for the perception that the environment was being modified, and

- Was seen as a positive action toward a better working environment.

As the research question suggests, the technology is seen as contributing and driving the force of the change in the environment. Many of the interviewees also have made this connection to their experiences. 
"And it's the technology that's driving that, you know. Nothing that any one boss has done or any charisma or that type of thing."20

"...one thing that's really bizarre that's come out of this PantherSoft thing is the realization that either the procedures we had were inefficient, didn't work, or we didn't even have any. So now the new software has so many different types of information, it's just so much more data in it, and it makes it so much more accessible, and organized, that it's forcing everyone to talk now, and realize we have to create new (procedures), and it's just funny to see how, like, to answer the question, is it changing, you know, and it is and it's funny to see it changing by accident, by necessity, rather than by someone's design."21

Returning to Schein's cultural model, visible manifestations of culture include language arts, architecture, technology, and other material outputs. These are the outward system of characteristics that assist in shaping and reinforcing the organizational behavioral norms. The inclusion of the technology as a structural element in this level regards the element as a regulating component. Material from the interviews suggests that the technology influence creeps into the other material outputs such as language arts.

"You change the system, you change the terminology, (and) you have changed the culture, basically." 22

The path analysis of the second measurement documents a different work environment than in the first measurement period. Is this a picture of organizational change in motion?

\section{Limitations}

The findings of this research study do have limitations. The first is that the study took place in one organization. The replication of the responses for the employees in

\footnotetext{
${ }^{20}$ From the interview with participant R1001 7/27/04.

${ }^{21}$ From the interview with participant R1001 7/27/04.

${ }^{22}$ From the interview with participant R1010, 7/23/04.
} 
another organization may change the association between variables. To validate the findings, the study should be replicated in another organization implementing a large ERP system. Also, with the absence of a control group, the results cannot be attributed solely to the variables studied. Other elements in the environment may be causing change that is reflective through the measured variable.

A second limitation is the use of the selected instruments. Although each was selected as the best tool available for the subject, the measurement is only as good as the survey being able to accurately capture individual responses. Any weakness in variable meaning would be magnified by the variability analysis.

Third, given the subject of the surveys, latent variables have not been included as potential impacts in the environment. These may include (but are not limited to) user training, department manager styles, and the self-understanding of one's job. This perceptual data - as opposed to quantifiable data - also may be considered a limitation. Individual perceptions are not a discrete variable and are able to be influenced and changed. The objective of this research, however, was to show that people perceive that technology, job, and organizational culture are significantly related to each other.

Finally, this research is not exhaustive. More variables could have been examined in each concept. Consequently, other variables may exist that are more significantly associated with the constructs than the ones identified in this research.

Future Directions

There are several possible future extensions of this research project that can be developed. The first would be to continue this project over the next year to document 
continuing change effects. Further evidence may introduce new organizational linkages among system, job, and culture characteristics. Second, a deeper understanding of the variables used in the study needs to be achieved. Organizational culture is more than a perception of co-worker behavior and, therefore, needs to be explored further. The system characteristics need to be adjusted to address the new technologies in the work environment and their impact on the job.

The interrelationship between information systems and sense-making should be addressed, as the findings indicated that sense-making is a major function in change for organizations. A better understanding of how this takes place and its impact at both the individual and group level will contribute to the understandings found in this study.

The issues surrounding a single case study are many; however, the overriding one is the ability of the study to be replicated in other environments. This study needs to be recreated in a different but similar environment to validate the findings.

Conclusions

As discussed in the organization change section, organizational transformations are an expected result of successful system implementations. The results of combining technology and organizations depend on a complex array of visible and invisible factors at all levels of the institution. The visible success of the implementation may be a better running work environment, while an invisible outcome may be the changed perspectives of the workers toward their jobs and their movement toward common beliefs. The use of technology as both a tool and a symbol of change forces members of organizations to view their surroundings in a new context. 
As in earlier studies (Orlikowski, 2000; Harper and Ultey, 2001), technology is seen as an occasion for change; however, its role as a planned support of culture is an aftereffect. The findings of this research project support the findings of these studies and extend the understanding of the role of technology in organizational change. The importance of this research is its establishment of the linkage between system characteristics and their influences on how people work. This is the first step in understanding the invisible effects of technology that have always been suggested but never confirmed.

In conclusion, this study has presented interesting results that lead to a number of interesting questions. Can system characteristics be used during a change to promote constructive behaviors? Is the linkage between system characteristics and job perception understood? How can system characteristics be changed to support the job task? Organizational culture is a homogenous term for common individual beliefs. In this research, the definition of culture has been limited to the behavioral dimensions defined by the $\mathrm{OCI}$ instrument. All behaviors are present in the environment but only a few emerge. Have individuals changed from their dominant behavioral style to under utilized styles to comprehend the new system? Are constructive behaviors simply a better way to fulfill their individual needs rather than using passive or aggressive behavior? After the need of the individual is met, will the dominant group behaviors return? If the environment had higher levels of constructive behaviors, would more group sensemaking activities be found?

This research has demonstrated that system characteristics of ERP do contribute to the perception of change in an organization and do support organizational culture 
behaviors and job characteristics. It has shed light on how information technology - as a cultural component - impacts the organization. 


\section{LIST OF REFERENCES}

Akin, G. and D. Hopelain (1986). "Finding the Culture of Productivity." Organizational Dynamics 7(2): 19-32.

Al-Mashari, M. and A. Al-Mudimigh (2003). "ERP Implementation: Lessons from a Case Study." Information Technology \& People 16(1): 21-33.

Allen, R. F. and F. J. Dyer (1980). "A Tool for Tapping The Organizational Unconscious." Personnel Journal 59: 192-198.

Anonymous (1996). Kodak's picture is changing. Strategic Direction: 8.

Anonymous (2001). Peter Brabeck. Business Week: 78.

Argyres, N. S. (1999). "The Impact of Information Technology on Coordination: Evidence from the B-2 "Stealth" Bomber." Organization Science 10(2): 162-180.

Ashkanasy, N. M., L. E. Broadfoot, et al. (2000). Questionnaire Measures of Organizational Culture. Handbook of Organizational Culture and Climate. N. M. Ashkanasy, C. P. M. Wilderom and M. F. Peterson. Thousand Oaks, CA, Sage Publications, Inc.

Attwell, P. and J. Rule (1984). "Computing and Organizations: What We Know and What We Don't Know." Communications of the ACM 27(12): 1184-1191.

Bailey, J. E. and S. W. Pearson (1983). "Development of a Tool Measuring and Analyzing Computer User Satisfaction." Management Science 29(5): 530-544.

Barker, T. and M. N. Frolick (2000). "ERP Implementation Failure: A Case Study." Information and Management 20(4).

Barley, S. R. (1986). "Technology as an Occasion for Structuring: Evidence from Observations for CT Scanners and Social Order of Radiology Departments." Administration Science Quarterly 31: 78-108.

Barley, S. R., G. W. Meyer, et al. (1988). "Cultures of Culture: Academics, Practitioners and the Pragmatics of Normative Control." Administrative Science Quarterly 33: 24-60.

Bingi, P., M. K. Sharma, et al. (1999). "Critical Issues Affecting An ERP Implementation." Information Systems Management: 7-14.

Blau, P. M. and R. A. Shoenherr (1971). The Structure of Organizations. New York, Basic Books. 
Buono, A. F., J. L. Bowditch, et al. (1985). "When Cultures Collide: The Anatomy of a Merger." Human Relations 38(5): 477-500.

Burke, W. W. and N. W. Biggart (1997). How Mergers Go Wrong. Enhancing Organizational Performance. D. Druckman, J. E. Singer and H. Van Cott. Washington, D.C., National Academy Press: 120-149.

Burrus-Barbey, K. (2000). Leadership, Global Management, and Future Challenges: An Interview with Peter Brabeck-Letmathe, Chief Executive Officer of Nestle SA. Thunderbird International Business Review. 42: 495-506.

Caldwell, B. (1998). Beyond ERP: New IT Agenda. InformationWeek. 711: 30.

Child, J. and R. Mansfield (1972). "Technology, Size and Organization Structure." Sociology 6: 369-393.

Clarke, J., S. Hall, et al. (1981). Subcultures, Cultures, and Class. Culture, Ideology and Social Process. T. Bennett, G. Martin, C. Mercer, and J. Wallacott. London, Batsford: 53-79.

Cook, M. (1996). Building Enterprise Information Architectures. Upper Saddle River, NJ, Prentice Hall.

Cooke, R. A. and D. M. Rousseau (1988). "Measuring Normative Beliefs and Shared Behavioral Expectations in Organizations: A Quantitative Approach to the Assessment of Organizational Culture." Group \& Organization Studies 13: 245 273.

Cooke, R. A. and J. L. Szumal (1993). "Measuring Normative Beliefs and Shared Behavioral Expectations in Organizations: The Reliability and Validity of the Organizational Culture Inventory." Psychological Reports (72): 1299-1330.

Cooke, R. A. and J. L. Szumal (2000). Using the Organizational Culture Inventory. Handbook of Organizational Culture and Climate. N. M. Ashkanasy, C. Wilderom and M. F. Peterson. Thousand Oaks, CA, Sage Publications, Inc.

Cowan, E. J. and L. B. Eder (2002). "The Transformation of AT\&T's Enterprise Network Systems Group to Avaya: Enabling the Virtual Corporation through Reengineering and Enterprise Resource Planning." Journal of Information Systems Education 14(3): 325-331.

Cummings, T. G. and C. G. Worley (1998). Organizational Development and Change. St. Paul, MN, West. 
Davis, F. D. (1989). "Perceived Usefulness, Perceived Ease of Use, and User Acceptance of Information Technology." MIS Quarterly 13(3): 319-339.

Davis, F. D. (1993). "User Acceptance of Information Technology: System Characteristics, User Perceptions and Behavioral Impacts." International Journal of Man-Machine Studies 38(3): 319-339.

Day, G. S. (1999). "Creating a Market-Driven Organization." Sloan Management Review 41(1): 11-22.

Deale, T. F. and A. A. Kennedy (1982). Corporate Culture: The Rites and Rituals of Corporate Life. Reading, MA, Addison-Wesley.

Denison, D. R. (1990). Corporate Culture and Organizational Effectiveness. New York, John Wiley.

Denison, D. R. (1996). "What is the Difference between Organizational Culture and Organizational Climate? A Native's Point of View on a Decade of Paradigm Wars." Academy of Management Review 21: 619-654.

Dunham, R. B., J. L. Pierce, et al., "Job Context and Job Content: A Conceptual Perspective." Journal of Management 9 (2):187-203.

Dwyer, G., L. Heralcleous, et al. (2003). "Grounding Strategic Change Practice in Theory: the Case of Avaya Ireland, 1992-2000." Strategic Change 12: 123-135.

Epstein, B. J. and W. R. King (1982). "An Experimental Study of the Value of Information." Omega 10(3): 249-258.

Fields, D. L. (2002). Taking the Measure of Work. Thousand Oaks, CA, Sage Publications, Inc.

Fleck, J. (1993). Configurations: Crystallizing Contingency. The International Journal of Human Factors in Manufacturing. New York, John Wiley \& Sons. 3.

Fombuan, C. J. (1983). "Corporate Culture, Environment and Strategy." Human Resources Management 22: 139-152.

Francesconi, T. (1998). Transforming Lucent's CFO. Management Accounting. 80: 2230.

Fry, L. W. and J. W. Slocum, Jr. (1984). "Technology, Structure, and Workgroup Effectiveness: A Test of a Contingency Model." Academy of Management Journal 21: 221-246. 
Gaucher, E. and E. Kratochwill (1993). "The Leader's Role in Implementing Total Quality Management." Quality Management in Health Care 1(3): 10-18.

Georgopoulos, B. S. (1996). Organizational Structure, Problem Solving, and Effectiveness. San Francisco, Jossey-Bass.

Gerwin, D. (1981). Relationships between Structure and Technology. Handbook of Organizational Design. P. C. Nystrom and W. H. Starbuck. New York, Oxford. 2: 3-38.

Goodhue, D. L. (1995). "Understanding User Evaluations of Information Systems." Management Science 41(12): 1827.

Goodhue, D. L. (1998). "Development and Measurement Validity of a Task-Technology Fit Instrument for User Evaluations of Information Systems." Decision Sciences 29(1): 105-138.

Gordon, G. G. and N. DiTomaso (1992). "Predicting Corporate Performance from Organizational Culture." Journal of Management Studies(29): 783-798.

Graves, D. (1986). Corporate Culture - Diagnosis and Change. London, Frances Pinter.

Haber, S. B., J. N. O'Brien, et al. (1991). Influence of Organizational Factors on Performance Reliability. Upton, NY: Brookhaven National Laboratory, U.S. Nuclear Regulatory Commission, Division of System Research.

Hackman, J. R. and G. R. Oldham (1974). The Job Diagnostic Survey: An instrument for the diagnosis of jobs and the evaluation of job redesign projects. New Haven, CT, Yale University, Department of Administration.

Hackman, J. R. and G. R. Oldham (1980). Work redesign. Reading, MA, AddisonWesley.

Hage, J. and M. Aiken (1967). "Program Change and Organizational Properties: A Comparative Analysis." American Journal of Sociological 72: 503-519.

Harper, G. and D. Utley (2001). "Organizational Culture and Successful Information Technology Implementation." Engineering Management Journal 13(2): 11.

Hickson, D. J., D. S. Pugh, et al. (1969). "Operations Technology and Organization Structure: An Empirical Reappraisal." Administration Science Quarterly 14: 378397. 
Hoffman, N. and R. Klepper (2000). "Assimilating New Technologies The role of Organizational Culture." Information Systems Management(Summer 2000): 36 42.

Hofstede, G. (1980). Culture's Consequences. International Differences in Work-Related Values. Beverly Hills, CA, Sage Publications.

Holsapple, C. W. and M. P. Sena (2005). "ERP Plans and Decision-Support Benefits." Decision Support Systems 38: 575-590.

Huber, G. P. (1990). "A Theory of the Effects of Advance Information Technologies on Organizational Design, Intelligence, and Decision Making." Academy of Management Journal 15(1): 47-71.

Idaszak, J. R. and F. Drasgow (1987). "A Revision of the Job Diagnostic Survey: Elimination of a Measurement Artifact." Journal of Applied Psychology 72(1): 69-74.

Kilmann, R. H. and M. J. Saxton (1983). The Kilmann-Saxton Culture-Gap Survey. Pittsburgh, PA, Organizational Design Consultants.

Kilmann, R. H. and T. J. Covin (1988). Introduction: Key Themes in Corporate Transformation. Corporate Transformation. R. H. Kilmann and T. J. Covin. San Francisco, Jossey-Bass Inc.: 1 - 16.

Koch, C. (1999). From the Team Techie to Enterprise Leader. $\underline{\text { CIO. }}$

Koch, C. (2001). "Enterprise Resource Planning Information Technology as a Steamroller for Management Politics?" Journal of Organizational Change Management 14(1): 64 -78.

Kotter, J. P. and J. L. Heskett (1992). Corporate Culture and Performance. New York, Free Press.

Kunda, G. (1992). Engineering Culture: Control and Commitment in High-Tech Corporations. Philadelphia, PA, Temple University Press.

Laurila, J. (1997). Discontinuous Technological Change as a Trigger for Temporary Reconciliation of Managerial Subcultures: A Case Study of a Finnish Paper Industry Company. Cultural Complexity in Organizations. S. A. Sackman. Thousand Oaks, CA, Sage Publications, Inc.

Lawler, E. E. (1988). Transformation from Control to Involvement. Corporate Transformation. R. H. Kilmann and T. J. Covin. San Francisco, Jossey-Bass Inc. 
Leavitt, H. and T. Whisler (1958). "Management in the '80's." Harvard Business Review: 41.

Leidner, D. E., S. Carlsson, et al. (1999). "Mexican and Swedish Managers' Perception of the Impact of EIS on Organizational Intelligence, Decision Making, and Structure." Decision Sciences 30(3): 633-657.

Lewin, K. (1951). Field Theory in Social Science. New York, Harper \& Row.

Likert, R. (1961). New Patterns of Management. New York, McGraw-Hill.

Lind, M. R. and R. W. Zmud (1991). "The Influence of a Convergence in Understanding Between Technology Providers and Users on Information Technology Innovativeness." Organization Science 2: 195-217.

Louis, A. M. (1982). The Bottom Line on 10 Big Mergers. Fortune: 84-89.

Lucas, H. C. J. and J. Baroudi (1994). "The Role of Information Technology in Organization Design." Journal of Management Information Systems 10(4): 9.

Manning, P. K. (1996). "Information Technology in the Police Context: The "Sailor" Phone." Information Systems Research 7(1): pp. 52 - 62.

Melone, N. P. (1990). "A Theoretical Assessment of the User-Satisfaction Construct in Information System Research." Management Science 36(1): 76-91.

Munz, D. C., T. J. Huelsman, et al. (1996). "Are There Methodological and Substantive Roles for Affectivity in Job Diagnostic Survey Relationships?" Journal of Applied Psychology 81(6): 795-805.

Meyer, G. W. and W.H. Starbuck (1993). "Interactions between Politics and Ideologies in Strategy Formation." New Challenges to Understanding Organizations. K. Roberts. New York, Macmillan: 99-116.

Orlikowski, W. J. (2000). "Using Technology and Constituting Structures: A Practice Lens for Studying Technology in Organizations." Organization Science 11(4): 404-428.

Orlikowski, W. J. and D. C. Gash (1994). "Technological frames: Making Sense of Information Technology in Organizations." ACM Transactions on Information Systems 12: 174-207.

Osterland, A. (2000). Blaming ERP. CFO Magazine. 
Ouchi, W. G. (1980). "Markets, Bureaucracies and Clans." Administrative Science Quarterly(25): 129-141.

Palaniswamy, R. and T. Frank (2000). "Enhancing Manufacturing Performance with ERP Systems." Information Systems Management 17(3): 43-55.

Peters, T. J. and R. H. Waterman (1982). In Search of Excellence: Lessons from America's Best-Run Companies. New York, Harper \& Row.

Pierce, J.L. and R. B. Dunham (1976) "Task Design: A Literature Review." Academy of Management Review 1: 83-97.

Pritchett, P. (1985). After the Merger. Homewood, IL, Dow Jones-Rwin.

Rajaagopal, P. (2002). "An Innovation-Diffusion View of Implementation of Enterprise Resource Planning (ERP) Systems and Development of a Research Model." Information and Management 40: 87-114.

Renn, R. W. and R. J. Vandenberg (1995). "The Critical Psychological States: An Underrepresented Component in Job Characteristics Model Research." Journal of Management 21(2): 279-303.

Robey, D. and S. Sahay (1996). "Transforming Work through Information Technology: A Comparative Case Study of Geographic Information Systems in County Government." Information Systems Research 7(1): 63-92.

Rousseau, D. M. (1990). Assessing Organizational Culture: The Case for Multiple Methods. Organizational Climate and Culture. B. Schneider. San Francisco, Jossey-Bass.

Sarkis, J. and R. P. Sundarraj (2003). "Managing Large-Scale Global Enterprise Resource Planning Systems: A Case Study at Texas Instruments." International Journal of Information Management 23: 431-442.

Sathe, V. (1985). Managing an Entrepreneurial Dilemma: Nurturing Entrepreneurship and Control in Large Corporations. Frontiers of Entrepreneurship Research. Wellesley, Mass, Babson College: 636-656.

Schein, E. H. (1981). "Does Japanese Management Style have a Message for American Managers?" Sloan Management Review 23(1): 55-68.

Schein, E. H. (1985). Organizational Culture and Leadership: A Dynamic View. San Francisco, CA. Jossey-Bass.

Scholz, C. (1987). "Corporate Culture and Strategy." Long Range Planning(20): 78-87. 
Shadish, W. R., Cook, T. D., et al. (2002). Experimental and Quasi-Experimental Designs for Generalized Causal Inference. Boston, MA. Houghton Mifflin Company.

Shurber, D. A. and S. B. Haber (1992). Organizational Culture During The Accident Response Process. Topical Meeting on Risk Management, La Grange Park, IL, American Nuclear Society.

Siegall, M. and T. McDonald (1995). "Focus of Attention and Employee Reactions to Job Change." Journal of Applied Social Psychology 25(13): 1121-1141.

Slowinski, G. (1992). "The Human Touch in Successful Strategic Alliances." Mergers and Acquisitions(27): 44-47.

Spector, P. E., S. Jex, et al. (1995). "Relations of Job Characteristics from Multiple Data Sources with Employee Affect, Absence, Turnover Intentions, and Health." Journal of Applied Psychology 76: 46-53.

Sprott, D. (2000). "Enterprise." Communications of the ACM 43(4): 63-69.

Steel, R. P. and J. R. Rentsch (1997). "The Dispositional Model of Job Attitudes Revisited: Findings of a 10-year Study." Journal of Applied Psychology 82(6): 873-879.

Stevens, T. (1997). Kodak focuses on ERP. Industry Week. 246: 130-135.

Stone, E.F. (1976) "The Moderating Effect of Work-Related Values on the Job ScopeJob Satisfaction Relationship." Organizational Behavior and Human Performance. 15 (2):147-155.

Stone, E. F., R. Mowday, et al. (1977). "Higher Order Need Strengths as Moderators of the Job Scope-Job Satisfaction Relationship." Journal of Applied Psychology 76: 46-53.

Swanson, E. B. (1987). "Information Channel Disposition and Use." Decision Sciences 18(1): 131-145.

Taber, T. D. and E. Taylor (1990). "A Review and Evaluation of the Psychometric Properties of the Job Diagnostic Survey." Personnel Psychology 43: 467-500.

Taylor, S. and P. A. Todd (1995). "Understanding Information Technology Usage: A Test of Competing Models." Information Systems Research 6(2): 144-176.

Trice, H. M. and J. M. Beyer (1984). "Studying Organizational Culture through Rites and Ceremonies." Academy of Management Review 9: 653-669. 
Turner, A. and D. Lawrence (1965). Industrial Jobs and the Worker. Cambridge, MA. Harvard University Press.

Weber, M. (1949). On the Methodology of the Social Science. Glencoe, IL, Free Press.

Weick, K. E. (1985). The Significance of Corporate Culture. Organizational culture. P. J. Frost, L. F. Moore, M. R. Louis, C. C. Lundberg and J. Martin. Beverly Hills, CA, Sage Publications, Inc.

Wheatley, M. (2000). ERP Training Stinks. CIO Magazine.

Woodward, J. (1965). Industrial Organizations: Theory and Practice. London, UK, Oxford University Press.

Worthen, B. (2002). Nestle's ERP Odyssey. CIO Magazine.

Xenikou, A. and A. Furnham (1996). "A Correlation and Factor Analytic Study of Four Questionnaires Measures of Organizational Culture." Human Relations 16(2): 4955 .

Yates, J. and J. Van Maanen (2001). Introduction. Information Technology and Organizational Transformation. J. Yates and J. Van Maanen. Thousand Oaks, CA, Sage Publications.

Yin, R. K. (1994). Case Study Research. Thousand Oaks, CA, Sage Publications, Inc.

Yusuf, Y., A. Gunasekaran, et al. (2004). "Enterprise Information Systems Project Implementation: A Case Study of ERP in Rolls-Royce." International Journal Production Economics 87: 251-266.

Zmud, R. W. (1978). "An Empirical Investigation of the Dimensionality of the Concept of Information." Decision Sciences 9(2): 187-195. 
H1 The perception of the information system's data detail will be positively associated with the worker's perception of job variety.

The perception of the information system's data detail will be positively associated with the worker's perception of job identify.

Yes

Yes

The perception of the information system's data detail will be positively associated with the worker's perception of autonomy.

$\begin{array}{ll}\text { Yes } & \text { Yes } \\ \text { No } & \text { Yes } \\ \text { Yes } & \text { Yes } \\ \text { Yes } & \text { Yes } \\ \text { No } & \text { Yes } \\ \text { Yes } & \text { No } \\ \text { Yes } & \text { No } \\ \text { No } & \text { Yes } \\ \text { Yes } & \text { No } \\ \text { No } & \text { No }\end{array}$

The perception of the information system's data detail will be positively associated with the worker's perception of feedback.

H5: The perception of the information system's data detail will be positively associated with the worker's perception of significance.

H6: The perception of the information system's data detail will be positively associated with the worker's perception of working with others.

H7: The perception of the information system's data accuracy will be positively associated with the worker's perception of job variety.

H8: The perception of the information system's data accuracy will be positively associated with the worker's perception of job identify.

H9: The perception of the information system's data accuracy will be positively associated with the worker's perception of autonomy.

H10: The perception of the information system's data accuracy will be positively associated with the worker's perception of feedback.

H11: The perception of the information system's data accuracy will be positively associated with the worker's perception of significance.

The perception of the information system's data accuracy will be

H12: positively associated with the worker's perception of working with others.

The perception of the information system's data compatibility will be

H13: positively associated with the worker's perception of job variety.

The perception of the information system's data compatibility will be

H14: positively associated with the worker's perception of job identify.

The perception of the information system's data compatibility will be

H15: positively associated with the worker's perception of autonomy.

The perception of the information system's data compatibility will be

H16: positively associated with the worker's perception of feedback.

The perception of the information system's data compatibility will be

H17: positively associated with the worker's perception of significance.

The perception of the information system's data compatibility will be positively associated with the worker's perception of working with

H18: others.

The perception of the information system's data location will be

H19: positively associated with the worker's perception of job variety.

The perception of the information system's data location will be

$\mathrm{H} 20$ : positively associated with the worker's perception of job identify. 
The perception of the information system's data location will be positively

$\mathrm{H} 21$ : associated with the worker's perception of autonomy.

The perception of the information system's data location will be positively

H22: associated with the worker's perception of feedback.

$\begin{array}{ll}\text { No } & \text { Yes } \\ \text { Yes } & \text { Yes } \\ \text { Yes } & \text { Yes }\end{array}$

$\mathrm{H} 23$ : associated with the worker's perception of significance.

The perception of the information system's data location will be positively

$\mathrm{H} 24$ : associated with the worker's perception of working with others.

No

No

The perception of the information system's data accessibility will be

$\mathrm{H} 25$ : positively associated with the worker's perception of job variety.

The perception of the information system's data accessibility will be

H26: positively associated with the worker's perception of job identify.

The perception of the information system's data accessibility will be

$\mathrm{H} 27$ : positively associated with the worker's perception of autonomy.

The perception of the information system's data accessibility will be

H28: positively associated with the worker's perception of feedback.

The perception of the information system's data accessibility will be

H29: positively associated with the worker's perception of significance.

The perception of the information system's data accessibility will be

H30 positively associated with the worker's perception of working with others.

The perception of the information system's data meaning will be positively

H31: associated with the worker's perception of job variety.

No No

The perception of the information system's data meaning will be positively

H32: associated with the worker's perception of job identify.

Yes

No

The perception of the information system's data meaning will be positively

H33: associated with the worker's perception of autonomy.

No

Yes

The perception of the information system's data meaning will be positively

H34: associated with the worker's perception of feedback.

Yes $\quad$ Yes

The perception of the information system's data meaning will be positively

H35: associated with the worker's perception of significance

No

Yes

The perception of the information system's data meaning will be positively

H36: associated with the worker's perception of working with others.

No No

The perception of the information system's data concurrency will be

H37: positively associated with the worker's perception of job variety.

The perception of the information system's data concurrency will be

H38: positively associated with the worker's perception of job identify.

The perception of the information system's data concurrency will be

H39: positively associated with the worker's perception of autonomy

The perception of the information system's data concurrency will be

$\mathrm{H} 40$ : positively associated with the worker's perception of feedback.

Yes $\quad$ Yes

Yes $\quad$ Yes

No Yes

Yes Yes 
The perception of the information system's data concurrency will be

$\mathrm{H} 41$ : positively associated with the worker's perception of significance.

The perception of the information system's data concurrency will be

Yes

Yes

positively associated with the worker's perception of working with

H42: others.

No

The perception of the information system's data assistance will be

H43: positively associated with the worker's perception of job variety.

The perception of the information system's data assistance will be

H44: positively associated with the worker's perception of job identify.

H45: The perception of the information system's data assistance will be positively associated with the worker's perception of autonomy.

The perception of the information system's data assistance will be

H46: positively associated with the worker's perception of feedback.

The perception of the information system's data assistance will be

H47: positively associated with the worker's perception of significance

The perception of the information system's data assistance will be positively associated with the worker's perception of working with

H48: others.

The perception of the information system's ease of use will be positively

H49: associated with the worker's perception of job variety.

The perception of the information system's ease of use will be positively

H50: associated with the worker's perception of job identify.

The perception of the information system's ease of use will be positively

H51: associated with the worker's perception of autonomy.

The perception of the information system's ease of use will be positively

H52: associated with the worker's perception of feedback.

The perception of the information system's ease of use will be positively

H53: associated with the worker's perception of significance.

$\begin{array}{ll}\text { No } & \text { No } \\ \text { Yes } & \text { Yes } \\ \text { Yes } & \text { No } \\ \text { No } & \text { No } \\ \text { No } & \text { Yes } \\ \text { No } & \text { No }\end{array}$

The perception of the information system's ease of use will be positively

H54: associated with the worker's perception of working with others.
The perception of the information system's reliability will be positively

No $\quad$ No

The perception of the information system's reliability will be positively

H55: associated with the worker's perception of job variety.

No $\quad$ No

The perception of the information system's reliability will be positively

H56: associated with the worker's perception of job identity.
The perception of the information system's reliability will be positively

No No

H57: associated with the worker's perception of autonomy.

No No

H58: The perception of the information system's reliability will be positively associated with the worker's perception of feedback.

No No

The perception of the information system's reliability will be positively

H59: associated with the worker's perception of significance.

No No

H60: The perception of the information system's reliability will be positively associated with the worker's perception of working with others.

No

No 
H61: The perception of the information system's presentation will be positively associated with the worker's perception of job variety.

$\begin{array}{cc}\text { Yes } & \text { Yes } \\ \text { Yes } & \text { No } \\ \text { No } & \text { Yes } \\ \text { Yes } & \text { Yes } \\ \text { No } & \text { Yes }\end{array}$

H66: The perception of the information system's presentation will be positively associated with the worker's perception of working with others.

H67: If the perception of the information system is confusing to the user, it will be negatively associated with the worker's perception of job variety.

H68: If the perception of the information system is confusing to the user, it will be negatively associated with the worker's perception of job identity.

H69: If the perception of the information system is confusing to the user, it will be negatively associated with the worker's perception of job autonomy.

H70: If the perception of the information system is confusing to the user, it will be negatively associated with the worker's perception of the job feedback.

H71: If the perception of the information system is confusing to the user, it will be negatively associated with the worker's perception of job significance.

H72: If the perception of the information system is confusing to the user, it will be negatively associated with the worker's perception of working with others. period.

H73: Job variety is positively associated with constructive norms (achievement, selfactualizing, humanistic-encouraging, and affiliate behaviors).

H74:

Job variety is negatively associated with passive/defensive norms (approval, conventional, dependent, and avoidance behaviors).

H76: Job identity is positively associated with constructive norms (achievement, self-actualizing, humanistic-encouraging, and affiliate behaviors). 
H77: Job identity is not found in cultures characterized by a: Legacy $\quad$ b: ERP passive/defensive norms (approval, conventional, dependent, and avoidance behaviors).

No

Yes

H78: Job identity is not found in cultures characterized by aggressive/defensive norms (oppositional, power, competitive, and perfectionist behaviors)

No No

H79: Task significance is positively associated with constructive norms (achievement, self-actualizing, humanistic-encouraging, and affiliate behaviors).

H80: Task significance is negatively associated with passive/defensive norms (approval, conventional, dependent, and avoidance behaviors)

H81: Task significance is negatively associated with aggressive/defensive norms (oppositional, power, competitive, and perfectionist behaviors).

No

No

H82: Job autonomy is positively associated with constructive norms (achievement, self-actualizing, humanistic-encouraging, and affiliate behaviors).

H83: Job autonomy is negatively associated with passive/defensive norms (approval, conventional, dependent, and avoidance behaviors).

H84: Job autonomy is negatively associated with aggressive/defensive norms (oppositional, power, competitive, and perfectionist behaviors).

Yes

No

No

No

Yes

No

No

No

Yes

No

H85: Job feedback is positively associated with constructive norms (achievement, self-actualizing, humanistic-encouraging, and affiliate behaviors).

H86: Job feedback is negatively associated with passive/defensive norms (approval, conventional, dependent, and avoidance behaviors).

Yes

No

H87: Job feedback is negatively associated with aggressive/defensive norms (oppositional, power, competitive, and perfectionist behaviors).

Yes

Yes

Job design perceptions and job perception linkages to

$3 \mathrm{H} 2$ : organizational culture dimensions found in the legacy time period

Yes

Yes will be different in the ERP time period. 
1980-1985

1986-1989

1989-1999

1999-2003

2000-2005
B.S., Business Administration Florida State University

Tallahassee, Florida

Anderson Consulting, Inc.

Burger King Corporation

Towers Perrin

Doctorate Candidate

Business Administration

Florida International University

Miami, Florida

\section{PUBLICATIONS AND PRESENTATIONS}

Clemmons, S. and Simon, S. J. (2001). Control and Coordination in Global ERP Configuration. Business Process Journal, 7(3), 205-215.

Clemmons, S. and Henry K. (May, 2005). Reducing Risk in the Enterprise: Proposal for a Hybrid Audit Expert System. Paper presented at the meeting of the International Conference on Enterprise Information Systems. 